\title{
Graph Theory of Capping Golden Clusters
}

\author{
Enos Masheija Rwantale Kiremire \\ Correspondence: Enos Masheija Rwantale Kiremire, Professor of Chemistry, University of Namibia. Hochland Park, P. \\ O. Box, 98013, Pelican Square, Tauben Glen, Windhoek, Namibia. E-mail: kiremire15@yahoo.com
}

Received: December 1, 2017 Accepted: January 23, 2018 Online Published: January 25, 2018

doi:10.5539/ijc.v10n1p87

URL: https://doi.org/10.5539/ijc.v10n1p87

\begin{abstract}
Skeletal numbers and their valences have been extremely useful in analyzing and categorizing clusters especially boranes, carbonyls, and Zintl ions. This approach is being extended to the analysis and categorization of golden clusters. The newly introduced concept of graphing will also be applied to the clusters. The capping symbol $\mathrm{Kp}=\mathrm{C}{ }^{\mathrm{Y}} \mathrm{C}[\mathrm{Mx}]$ which has been restricted to post-closo clusters will be adapted for pre-closo cluster series. The concept of the existence of black holes in the nuclei of capping golden clusters will be introduced and explained.
\end{abstract}

Keywords: golden clusters, black holes, capping series, de-capping series, skeletal numbers, K(n) series, nuclearity index

\section{Introduction}

The first golden cluster to be synthesized was $\mathrm{Au}_{11} \mathrm{I}_{3} \mathrm{~L}_{7}$ in 1968(Mingos, 1984). Since then many more clusters have been synthesized(Cotton \&Wilkinson, 1980; Greenwood\&Earnshaw,1998;Gimeno,2008;Kilmartin, 2010;Kwok-Ming, 2011, Konishi, 2014). The golden clusters have attracted immense interest among many scientists in terms of trying to understand their geometrical shapes, bonding and potential applications in catalysis and nanotechnology(Corti, et al, 2005; Zhang, et al, 2014). The fascinating shapes of gold have been described by a number of ways, for instance, $\mathrm{Au}_{4} \mathrm{I}_{2} \mathrm{~L}_{4}$, tetrahedral; $\mathrm{Au}_{6} \mathrm{~L}_{6}{ }^{2+},\left(\mathrm{L}=\mathrm{PPh}_{3}\right)$, octahedral; $\mathrm{Au}_{8} \mathrm{~L}_{8}{ }^{2+}$, chair-like centered hexagon; $\mathrm{Au}_{13} \mathrm{Cl}_{2} \mathrm{~L}_{10}{ }^{3+},\left(\mathrm{L}^{3}=\mathrm{PMe}{ }_{2} \mathrm{Ph}\right)$; icosahedron; $\mathrm{Au}_{8} \mathrm{~L}_{7}{ }^{2+}$ and $\mathrm{Au}_{9} \mathrm{~L}_{8}{ }^{3+}$; flat, ring, torus or doughnut-shaped( Cotton \& Wilkinson, 1980;Greenwood \& Earnshaw, 1998). Further descriptions include, centered polyhedral, non-centered polyhedral and exo-attached polyhedral clusters(Konishi,2014). The recent application of the $4 \mathrm{n}$ series method to the analysis and categorization of golden clusters indicated almost all the clusters with nuclearity index 4 and above belong to the CAPPING SERIES (Kiremire, 2016, 2017). With the development of skeletal numbers and the concept of graph theory of capping series (Kiremire, 2017b, 2017c), it will be interesting to investigate how the golden cluster structures look like under the series graph theory.

\section{Results and Discussion}

In order to be able to compare and contrast the structures of gold as compared to other clusters, let us compare and contrast the structures of two hypothetical clusters, namely $\mathrm{C}_{6} \mathrm{H}_{10}$ and $\mathrm{Au}_{6} \mathrm{H}_{10}$. The $\mathrm{K}$ value of $\mathrm{C}_{6} \mathrm{H}_{10}$ : $\mathrm{K}=6[2]-5=7 ; \mathrm{K}(\mathrm{n})$ $=7(6), \mathrm{S}=4 \mathrm{n}+10$. In the case of $\mathrm{Au}_{6} \mathrm{H}_{10}: \mathrm{K}=6[3.5]-5=16 ; \mathrm{K}(\mathrm{n})=16(6), \mathrm{S}=4 \mathrm{n}-8, \mathrm{Kp}=\mathrm{C}^{5} \mathrm{C}[\mathrm{M} 1]$. The calculation of skeletal linkages of clusters has been discussed extensively (Kiremire, 2017d). Let us analyze these cases one at a time.

Ex-1: $\mathrm{C}_{6} \mathrm{H}_{10}: \mathrm{K}=6[2]-5=7 ; \mathrm{K}(\mathrm{n})=7(6), \mathrm{S}=4 \mathrm{n}+10$. The symbol $\mathrm{K}(\mathrm{n})=7(6)$ means that the 6 carbon skeletal atoms in the formula are joined by 7 linkages or bonds. One of the possible skeletal isomers is shown as F-1. The skeletal number of carbon $[\mathrm{C}], \mathrm{K}=2$, gold, $[\mathrm{Au}], \mathrm{K}=3.5,[\mathrm{H}]$ as a ligand, $\mathrm{K}=-0.5$ since it is a 1 electron donor (refer to appendixes 1 and 2). The number of valence electrons can also be derived from the series formula: $\mathrm{Ve}=4 \mathrm{n}+10=4(6)$ $+10=34$. This figure can be confirmed from the cluster formula itself: $\mathrm{VF}=6[4]+10=34$.

Ex-2: $\mathrm{Au}_{6} \mathrm{H}_{10}: \mathrm{K}=6[3.5]-5=16, \mathrm{~K}(\mathrm{n})=16(6), \mathrm{S}=4 \mathrm{n}-8, \mathrm{Kp}=\mathrm{C}^{5} \mathrm{C}[\mathrm{M} 1]$. This symbol means that the cluster is capped in such a way that 5 skeletal elements are surrounding 1 central skeletal atom at the nucleus. In addition, the skeletal framework of 6 golden elements is bound by 16 linkages. Since each capping skeletal element uses 3 linkages, the 5 capping elements means utilizing 15 of the skeletal linkages out of the 16. Since the cluster has one skeletal element $\{[M 1] ; n=1\}$, then the element obeys the CLOSO series formula $S=4 n+2$ and $K=2 n-1=2(1)-1=1$. Thus, the skeletal element in the nucleus utilizes 1 skeletal element and the 5 capping atoms consume 15 skeletal elements giving us a total of $15+1=16$. For categorization, we can use $S=4 n+q$ for main group and transition elements. However for calculating the number of valence electrons we have to use $\mathrm{Ve}=14 \mathrm{n}+\mathrm{q}$ equation. In this case, the number of valence electrons will be given by $\mathrm{Ve}=14 \mathrm{n}-8=14(6)-8=76$. We can verify this from the cluster formula $\mathrm{F}=\mathrm{Au}_{6} \mathrm{H}_{10} ; \mathrm{VF}=$ 
$6[11]+10=76$. Since $\mathrm{Kp}=\mathrm{C}^{5} \mathrm{C}[\mathrm{M} 1]$, the capping symbol can guide us to sketch a skeletal isomeric structure, F-2.

Ex-3: $\mathrm{Zn}_{6} \mathrm{H}_{10}: K=6[3]-5=13 ; \mathrm{K}(\mathrm{n})=13(6), \mathrm{S}=4 \mathrm{n}-2, \mathrm{Kp}=\mathrm{C}^{2} \mathrm{C}[\mathrm{M} 4]$. This is a bi-capped tetrahedron. Its possible isomeric structure has been proposed F-3.

Ex-4: $B_{6} H_{10}: K=6[2.5]-5=10 ; K(n)=10(6), S=4 n+4(q=4)$. The closo series is given by $S=4 n+2(q=2)$. Since the ordinary series, q runs in the multiples of $2, S=4 n+4$ is a step before $S=4 n+2$. If we follow the Rudolph type of capping series, then the closo above $\mathrm{B}_{6} \mathrm{H}_{10}$ will have a $\mathrm{K}$ value of 13 and $\mathrm{n}=7$. Hence, its closo cluster parameter will be $K(n)=13(7)=[M 7]$. Since capping clusters after [M7] are expressed as $K p=C^{y} C[M x]$, then the de-capping series can be expressed as $\mathrm{Kp}=\mathrm{C}^{-\mathrm{Y}} \mathrm{C}[\mathrm{Mx}]$. Therefore, the cluster $\mathrm{K}(\mathrm{n})=10(6)$, can be expressed as $\mathrm{Kp}=\mathrm{C}^{-1} \mathrm{C}[\mathrm{M} 7]$. According to this approach, this symbol means, its $\mathrm{K}(\mathrm{n})$ is one step below the closo base, that is, $\Delta \mathrm{K}=-3$, and $\Delta \mathrm{n}=-1$ $\equiv \Delta \mathrm{K}(\Delta \mathrm{n})=-3(-1)$. We can express this idea diagrammatically, SC-1. This means that by comparing the $\mathrm{q}$ value of a cluster and that of the 'standard reference' closo system, we can determine the relative positions of the K(n) values and hence numerically categorize the cluster, in this case $10(6), \mathrm{Kp}=\mathrm{C}^{-1} \mathrm{C}[\mathrm{M} 7]$.

Ex-5: $\mathrm{B}_{5} \mathrm{H}_{9}$ and Ex-6: $\mathrm{B}_{4} \mathrm{H}_{10}$ were numerically categorized in the same way.

$$
\begin{aligned}
& S=4 n+q \\
& \begin{array}{ccc}
C^{-1} C[M 7] & \Delta K(\triangle n)=3(1) & {[M 7]} \\
S=4 n+4 & 13(7) \\
S K(\Delta n)=-3(-1) & 4 n+2
\end{array} \\
& \begin{array}{c}
n=6 \\
q=4 \longrightarrow q=2
\end{array} \\
& \text { SC-1 }
\end{aligned}
$$
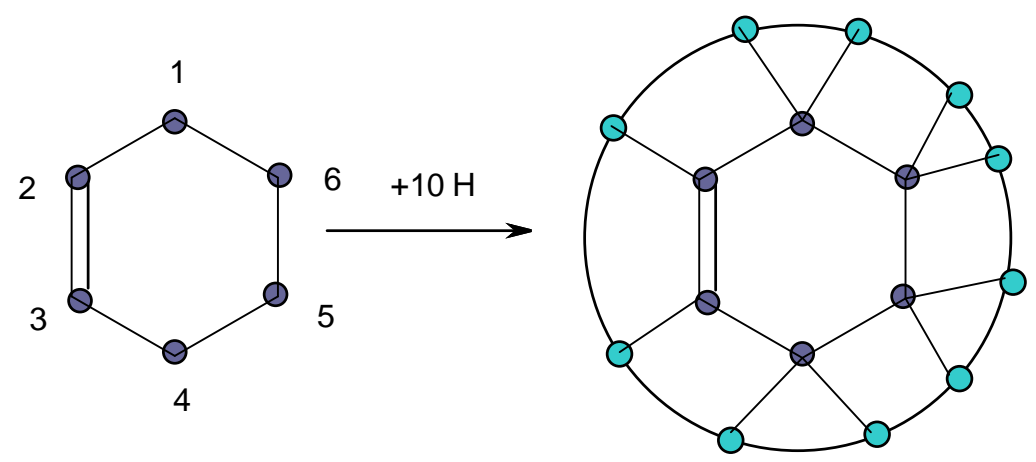

$\mathrm{K} 1=2=1=1=2 \mathrm{H}$

$\mathrm{K} 2=2-1.5=0.5=1 \mathrm{H}$

$\mathrm{K} 3=2-1.5=0.5=1 \mathrm{H}$

$\mathrm{K} 4=2-1=1=2 \mathrm{H}$

$\mathrm{K} 5=2-1=1=2 \mathrm{H}$

$\mathrm{K} 6=2-1=1=2 \mathrm{H}$

$$
\begin{aligned}
& O=\mathrm{C} \\
& \mathrm{O}=\mathrm{H}
\end{aligned}
$$

Ex-1: $\mathrm{C}_{6} \mathrm{H}_{10}$

Without Hydrogen Ligands 
Is $K(n)=10(6)$ below [M7]? We can test this by a simple check on the flow of $K(n)$ numbers.

[M7] means, $S=4 n+2(n=7)$. Hence $K=2 n-1=2(7)-1=13$ and so $K(n)=13(7)$ for [M7].

$[\mathrm{M} 7]$ lower series will be as follows starting with $[\mathrm{M} 7]=13(7) \rightarrow 10(6) \rightarrow 7(5) \rightarrow 4(4) \rightarrow 1(3)$. According to the historical terminology 13(7) will correspond to a CLOSO system, 10(6), NIDO; 7(5), ARACHNO; 4(4), HYPHO; and 1(3), KLADO system. The symbol [M7] will also represent the borane cluster $\mathrm{B}_{7} \mathrm{H}_{7}{ }^{2-}$. Hence, $\mathrm{K}(\mathrm{n})=10(6)$ is just one step below the closo system [M7] and corresponds to a nidoborane cluster $\mathrm{B}_{6} \mathrm{H}_{10}$.

According to the series method, $K(n)=16(6), K p=C^{5} C[M 1]$. This means it belongs to the group or a clan of clusters which have got 1 skeletal element in the nucleus. Thus, $K(n)=16(6)$ parameter will be found to be a member of [M1] based numbers. This can be seen to be the case as indicated in the [M1] series.

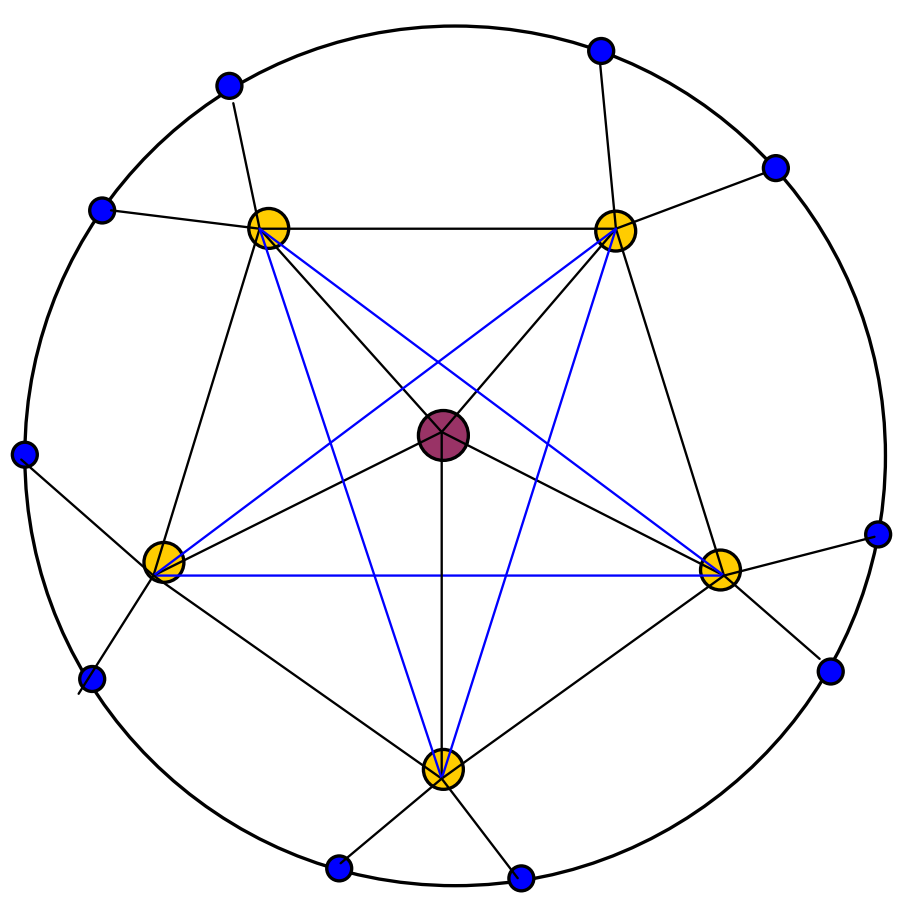


$\mathrm{Zn}_{6} \mathrm{H}_{10}: \mathrm{K}=6[3]-5=13 ; \mathrm{K}(\mathrm{n})=13(6), \mathrm{S}=4 \mathrm{n}-2, \mathrm{Kp}=\mathrm{C}^{2} \mathrm{C}[\mathrm{M} 4]$

$\mathrm{Ve}=14 \mathrm{n}-2=14(6)-2=82, \mathrm{VF}=6[12]+10=82$

[M4], $K=2 n-1=2(4)-1=7$

3
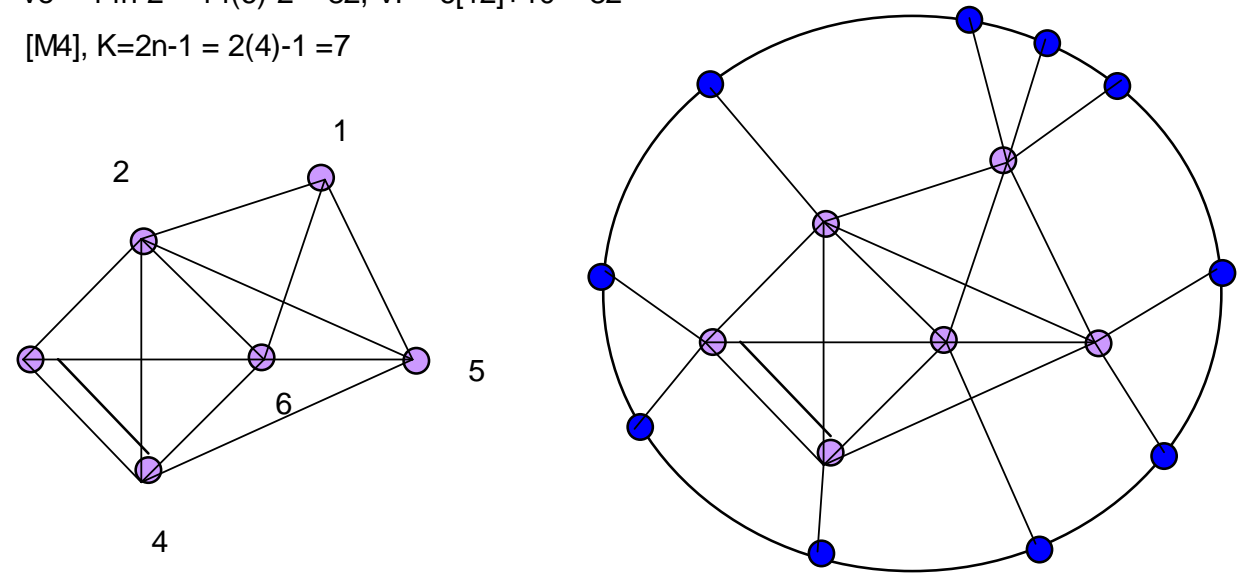

$\mathrm{K} 1=1[3]-1.5=1.5=3 \mathrm{H}$

$\mathrm{K} 2=1[3]-2.5=0.5=1 \mathrm{H}$

$\mathrm{K} 3=1[3]-2=1=2 \mathrm{H}$

$\mathrm{K} 4=1[3]-2.5=0.5=1 \mathrm{H}$

$\mathrm{K} 5=1[3]-2=1=2 \mathrm{H}$

$\mathrm{K} 6=1[3]-2.5=0.5=1 \mathrm{H}$

Is $K(n)=13(6)$ a member of [M4] series of numbers?. Let us check it out.

$K(n)=13(6)\left\{S=4 n-2, K p=C^{2} C[M 4]\right\} \rightarrow 10(5)\left\{S=4 n+0, K p=C^{1} C[M 5]\right\} \rightarrow 7(4)\{S=4 n+2\}$.The parameter $K(n)=7(4)$ $=[\mathrm{M} 4]$.

$$
\begin{aligned}
& \begin{array}{lll} 
& 5 & 9 \\
\mathrm{~B}_{5} \mathrm{H}_{9} & 2.5 & -0.5
\end{array} \\
& \underset{n=5}{S=4 n+4} \stackrel{\triangle_{q=2}}{\triangle_{n=1}}{ }_{n=6} n+2 \\
& \begin{array}{lll}
\mathrm{B}_{4} \mathrm{H}_{10} & 4 & 10 \\
2.5 & & 0.5
\end{array} \\
& \longrightarrow=4[2.5]-5=5 \\
& \mathrm{~K}(\mathrm{n})=5(4) \longrightarrow \mathrm{Kp}=\mathrm{C}^{-2} \mathrm{C}[\mathrm{M} 6] \\
& S=4 n+6 \\
& \mathrm{Ve}=4(4)+6=22 \\
& \mathrm{VF}=4[3]+10=22 \\
& \mathrm{~K}(\mathrm{n})=8(5) \longrightarrow \mathrm{Kp}=\mathrm{C}^{-1} \mathrm{C}[\mathrm{M} 6] \\
& \mathrm{Ve}=4(5)+4=24 \\
& \mathrm{VF}=5[3]+9=24 \\
& \text { [M6] CLAN SERIES } \\
& \triangle_{q=4} \\
& S=4 n+6 \\
& \triangle_{n=2} \\
& S=4 n+2 \\
& \mathrm{C}^{0} \mathrm{C}[\mathrm{M} 6] \\
& \mathrm{n}=4 \\
& \text { [M6] } \\
& \mathrm{n}=6 \\
& \text { [M6] CLAN SERIES } \\
& \mathrm{C}^{-1} \mathrm{C}[\mathrm{M} 6] \quad \mathrm{C}^{-2} \mathrm{C}[\mathrm{M} 6]
\end{aligned}
$$

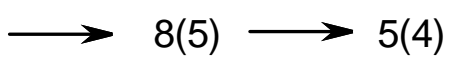




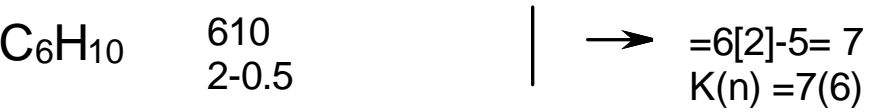

$$
\begin{aligned}
& S=4 n+10 \\
& S=4 n+10 \\
& \triangle q=8 \\
& n=6 \\
& S=4 n+2 \\
& \triangle_{n=4} \\
& \mathrm{n}=10
\end{aligned}
$$

[M10]; $S=4 n+2, K=2 n-1=2(10)-1=19, K(n)=19(10)$

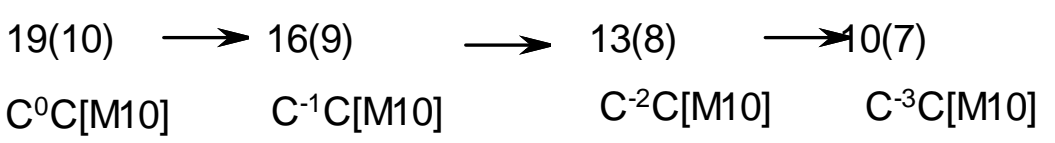

\section{SC-2}

The flow of K(n) numbers: the clan series (and families)

Since the main group and transition metal elements have been assigned skeletal numbers, this means that any cluster small or large that comprises these elements can naturally be assigned a cluster number parameter $\mathrm{K}(\mathrm{n})$ and hence be categorized. Take the examples:

1-1: MOTRIN - PAIN KILLER, FORMULA: $\mathrm{C}^{13} \mathrm{H}_{18} \mathrm{O}_{2}$; in this example, [C] and [O] are regarded as skeletal elements and $[\mathrm{H}]$ as a ligand. Its categorization is given in $\mathrm{SC}-3$

\section{$\mathrm{C}_{13} \mathrm{H}_{18} \mathrm{O}_{2} \quad$ MOTRIN $\longrightarrow$ PAIN KILLER}

$\mathrm{C}, \mathrm{O}$ are skeletal elements and $\mathrm{H}$ is a ligand. Hence, $K=13[2]+2[1]-18(0.5)=19$ $\mathrm{K}(\mathrm{n})=19(15)$

$S=4 n+22$ $S=4 n+2$

GENERAL SERIES, $S=4 n+q$

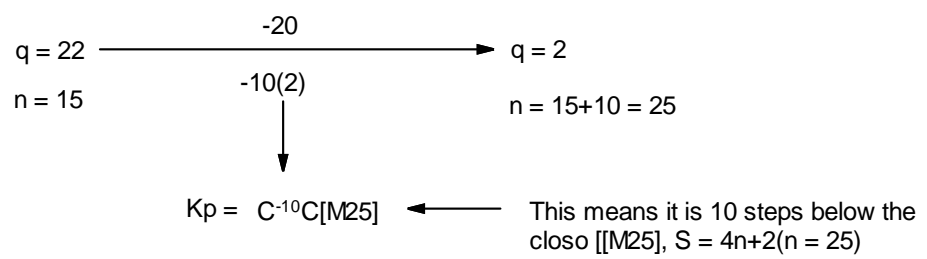

[M25]; $S=4 n+2 ; K=2 n-1=2(25)-1=49, K(n)=49(25)$

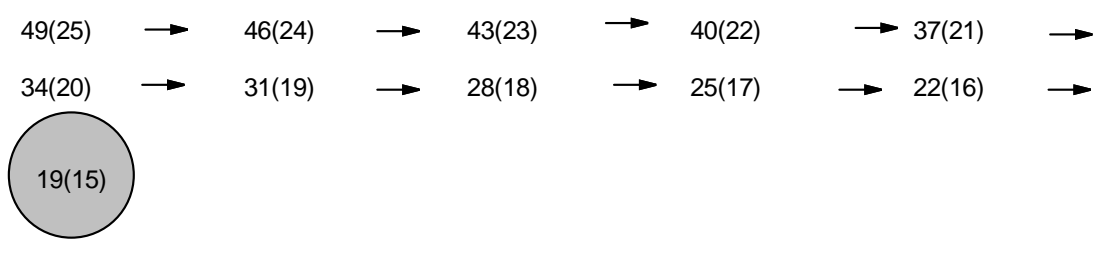




\section{1-2: ASPIRIN- PAIN KILLER, FORMULA, $\mathrm{C}_{9} \mathrm{H}_{8} \mathrm{O}_{4}$}

SKELETAL ELEMENTS, [C], [O] AND [H] LIGAND

$$
\begin{aligned}
& K=9[2]+4[1]-8(0.5)=18 \\
& K(n)=18(13) \\
& S=4 n+16(n=13)
\end{aligned}
$$

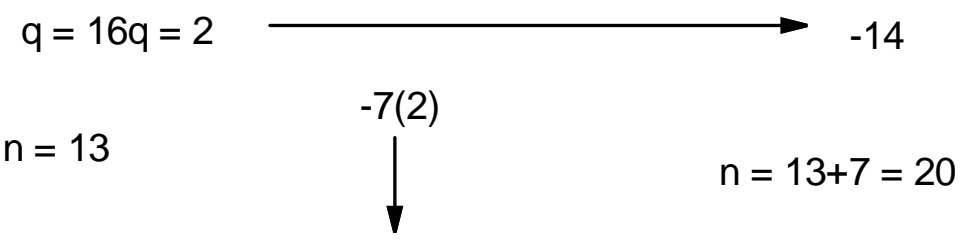

\section{$\mathrm{Kp}=\mathrm{C}^{-7} \mathrm{C}[\mathrm{M} 20] \longleftarrow$ This means the cluster is 7 steps below [M20] CLOSO SYSTEM.}

[M20], $\mathrm{K}=2 \mathrm{n}-1=2(20)-1=39, \mathrm{~K}(\mathrm{n})=39(20) \rightarrow 36(19) \rightarrow 33(18) \rightarrow 30(17) \rightarrow 27(16) \rightarrow 24(15) \rightarrow 21(14) \rightarrow 18(13)$

SC-4

1-3:QUININE - $\mathrm{C}_{20} \mathrm{H}_{24} \mathrm{~N}_{2} \mathrm{O}_{2}$

In this example, let us consider $[\mathrm{H}]$ as a ligand and the rest as skeletal elements. Its $\mathrm{K}$ value is given by $\mathrm{K}=20[2]+2[1.5]+2[1]-24(0.5)=33 ; \mathrm{K}(\mathrm{n})=33(24), \mathrm{S}=4 \mathrm{n}+30$. In this case $\mathrm{q}=30$ and we have to compare this with $\mathrm{q}=$ 2 for a closo system. That is,

$\mathrm{q}=30 \rightarrow \mathrm{q}=2$. The gap is 28 or we must subtract 28 from 30 to arrive at $\mathrm{q}=2$. Hence $\Delta \mathrm{q}=-28=-14(2)$. In terms of series, it means we have to go down numerically by 14 steps corresponding to an increase in $\mathrm{n}$ value by 14 . Hence, the new $n=24+14=38$. This means the CLOSO system will correspond to $n=38 \rightarrow[M 38]$. Similarly, the quinine formula itself will be at 14 steps below the [M38] closo system. Therefore its symbol is appropriately set at $\mathrm{Kp}=\mathrm{C}^{-14} \mathrm{C}[\mathrm{M} 38]$. We can test this from the flow of [M38] K(n) numbers, SC-5;

$[\mathrm{M} 38] \rightarrow 75(38) \rightarrow 72(37) \rightarrow 69(36) \rightarrow 66(35) \rightarrow 63(34) \rightarrow 60(33) \rightarrow 57(32) \rightarrow 54(31) \rightarrow 51(30) \rightarrow 48(29) \rightarrow 45(28) \rightarrow 42(27) \rightarrow 3$ $9(26) \rightarrow 36(25) \rightarrow \mathbf{3 3}(\mathbf{2 4})$

\section{SC-5}

The rest of the examples we can just demonstrate the categorization and the flow of K(n) number series.

1-4: $\mathrm{Cp}_{4} \mathrm{Ni}_{4} \mathrm{~B}_{4} \mathrm{H}_{4} ; \mathrm{K}=4[4]+4[2.5]-4(2.5)-4(0.5)=14, \mathrm{~K}(\mathrm{n})=14(8), \mathrm{S}=4 \mathrm{n}+4 ; \mathrm{q}=4 \rightarrow \mathrm{q}=2$. This means, the cluster is one step below the CLOSO system. Hence, $\mathrm{Kp}=\mathrm{C}^{-1} \mathrm{C}[\mathrm{M} 9]$. This is a NIDO cluster.

1-5: $\mathrm{Al}_{77} \mathrm{R}_{10}{ }^{-}: \mathrm{K}=77[2.5]-5-0.5=187 ; \mathrm{K}(\mathrm{n})=187(77), \mathrm{S}=4 \mathrm{n}-66, \mathrm{Kp}=\mathrm{C}^{34} \mathrm{C}[\mathrm{M} 43]$. This is a huge cluster with a large nucleus of 43 skeletal elements capped by 34 skeletal elements. Now, let us see the flow of the K(n) numbers downwards,SC-6.

$187(77) \rightarrow 184(76) \rightarrow 181(75) \rightarrow 178(74) \rightarrow 175(73) \rightarrow 172(72) \rightarrow 169(71) \rightarrow 166(70) \rightarrow 163(69) \rightarrow 160(68) \rightarrow 157(67) \rightarrow 154(6$ $6) \rightarrow 151(65) \rightarrow 148(64) \rightarrow 145(63) \rightarrow 142(62) \rightarrow 139(61) \rightarrow 136(60) \rightarrow 133(59) \rightarrow 130(58) \rightarrow 127(57) \rightarrow 124(56) \rightarrow 121(55) \rightarrow 1$ $18(54) \rightarrow 115(53) \rightarrow 112(52) \rightarrow 109(51) \rightarrow 106(50) \rightarrow 103(49) \rightarrow 100(48) \rightarrow 97(47) \rightarrow 94(46) \rightarrow 91(45) \rightarrow 88(44) \rightarrow \mathbf{8 5}(\mathbf{4 3})$

$\mathrm{K}(\mathrm{n})=85(43), \mathrm{S}=4 \mathrm{n}+2$; CLOSO SYSTEM. Hence, $\mathrm{K}(\mathrm{n})=187(77) \rightarrow \mathrm{Kp}=\mathrm{C}^{34} \mathrm{C}[\mathrm{M} 43]$ is in line with the $\mathrm{K}(\mathrm{n})$ number series.

SC-6

1-6: $\mathrm{Sb}_{7} \mathrm{Ni}_{3}(\mathrm{CO})_{3}{ }^{3-}: \mathrm{K}=7\left[1.5+3[4]-3-1.5=18 ; \mathrm{K}(\mathrm{n})=18(10), \mathrm{S}=4 \mathrm{n}+4 ; \mathrm{Kp}=\mathrm{C}^{-1} \mathrm{C}[\mathrm{M} 11]\right.$. This is a NIDO cluster $-\mathrm{a}$ derivative from [M11] which corresponds to $\mathrm{B}_{11} \mathrm{H}_{11}{ }^{2-}$ closo cluster.

1-7: $\mathrm{Rh}_{13} \mathrm{H}_{3}(\mathrm{CO})_{24}{ }^{2-}: \mathrm{K}=13[4.5]-1.5-24-1=32 ; \mathrm{K}(\mathrm{n})=32(13), \mathrm{S}=4 \mathrm{n}-12, \mathrm{Kp}=\mathrm{C}^{7} \mathrm{C}[\mathrm{M} 6]$. Let us look at the flow of the $\mathrm{K}(\mathrm{n})$ numbers and then the skeletal graph of the cluster.

$32(13) \rightarrow 29(12) \rightarrow 26(11) \rightarrow 23(10) \rightarrow 20(9) \rightarrow 17(8) \rightarrow 14(7) \rightarrow \mathbf{1 1}(\mathbf{6})$ 
This clearly agrees with the flow of the $\mathrm{K}(\mathrm{n})$ numbers and with the [M6] series below. The cluster symbol $\mathrm{Kp}=$ $\mathrm{C}^{7} \mathrm{C}[\mathrm{M} 6]$ means that the overall cluster has an $\mathrm{O}_{\mathrm{h}}$ nucleus of 6 skeletal elements surrounded by 7 capping skeletal elements. The sketch of the possible isomeric skeletal graph F-5

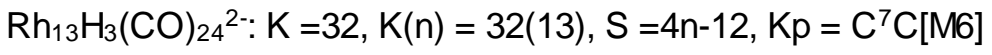

$$
\begin{aligned}
& \operatorname{Rh}(\mathrm{K}=4.5, \mathrm{~V}=9) \\
& \text { [M6], } K=2 n-1=2(6)-1=11 \\
& \text { SKELETAL LINKAGES }=7+7+7+[\mathrm{M} 6] \quad=21+11=32
\end{aligned}
$$

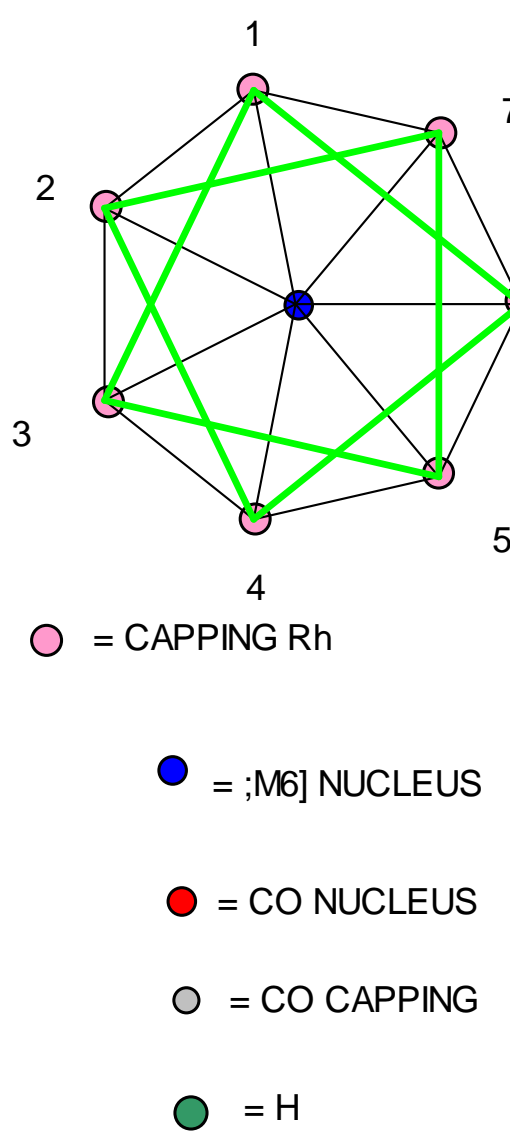

$\mathrm{K} 1=4.5-2.5=2$

$\mathrm{K} 2=\mathrm{K} 1=2$

$\mathrm{K} 3=\mathrm{K} 1=2$

$\mathrm{K} 4=\mathrm{K} 1=2$

$\mathrm{K} 5=\mathrm{K} 1=2$

$\mathrm{K} 6=\mathrm{K} 1=2$

$\mathrm{K} 7=\mathrm{K} 1=2$

$\mathrm{KN}=6[4.5]-11-7(0.5)-1=11.5$

$\mathrm{KT}=25.5=24 \mathrm{CO}+3 \mathrm{H}$

5

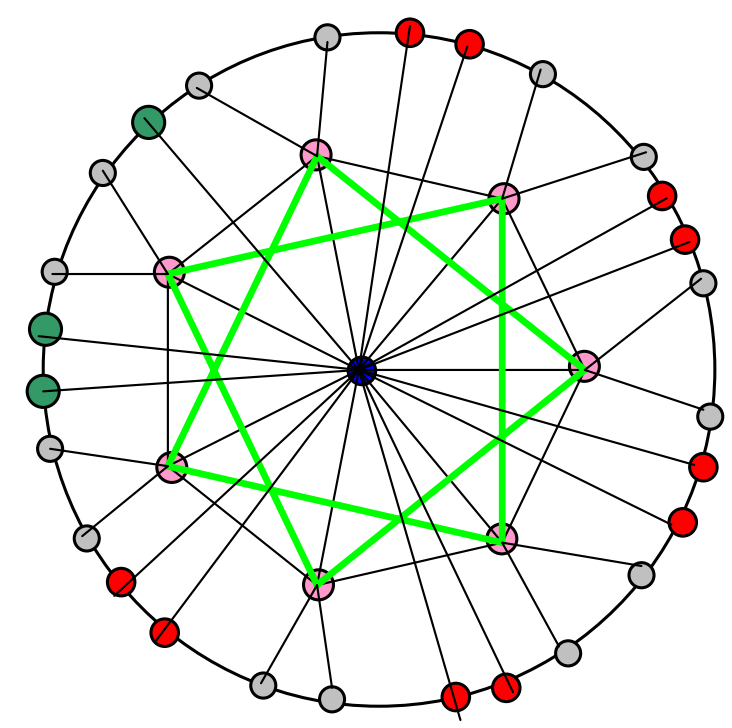

When we analyze more closely, it is quite clear that chemical elements, fragments, molecular formulas and clusters comprising of the main group and transition metals can readily be categorized using skeletal numbers. Therefore the clusters such as boranes, carboranes, metalloboranes, transition metal carbonyls, Zintl ion clusters and others all belong to one universe of clusters which obey a simple cluster formula $S=4 n+q(q>0$, non-capping clusters; $q \leq 0$, capping clusters) and a simple parameter K(n). It has been proposed that the clusters are best grouped according to the CLOSO NUCLEAR SYSTEM [Mx]. Although the golden clusters are usually centered around the closo nuclei [M1] and [M2] series, a number of examples of [Mx] series have been provided to allow readers to have familiarity with the beautiful flow of K(n) numbers. The important Rudolph correlation system set up about 40 years ago is a subset of the universe of K(n) numbers. A sample of selected [Mx] series SRS -1 are given below for illustrations and a selected collection of golden clusters are analyzed using skeletal numbers and their skeletal graphs mixed with a few non-golden clusters for comparison are shown F-6 to F-34

[M0]Series: 
$-1(0) \rightarrow 2(1)$

$\rightarrow 5(2) \rightarrow 8(3) \rightarrow 11(4) \rightarrow 14(5) \rightarrow 17(6) \rightarrow 20(7) \rightarrow 23(8) \rightarrow 26(9) \rightarrow 29(10) \rightarrow 32(11) \rightarrow 35(12) \rightarrow 38(13) \rightarrow 41(14) \rightarrow 44(15) \rightarrow 4$

$7(16) \rightarrow 50(17) \rightarrow 53(18) \rightarrow 56(19) \rightarrow 59(20) \rightarrow 62(21) \rightarrow 65(22) \rightarrow 68(23) \rightarrow 71(24) \rightarrow 74(25) \rightarrow 77(26) \rightarrow 80(27), \ldots$

BELOW [M0]

NEGATIVE K(n) VALUES

[M1]Series:1(1) $\rightarrow 4(2) \rightarrow 7(3) \rightarrow 10(4) \rightarrow 13(5) \rightarrow 16(6) \rightarrow 19(7) \rightarrow 22(8) \rightarrow 25(9) \rightarrow 28(10) \rightarrow 31(11) \rightarrow 34(12) \rightarrow 37(13) \rightarrow 40($ $14) \rightarrow 43(15) \rightarrow 46(16) \rightarrow 49(17) \rightarrow 52(18) \rightarrow 55(19) \rightarrow 58(20) \rightarrow 61(21) \rightarrow 64(22) \rightarrow 67(23) \rightarrow 70(24), \ldots \ldots .$.

BELOW [M1]

NEGATIVE K(n) VALUES

[M2] Series:

$\mathbf{3 ( 2 )} \rightarrow \mathbf{6 ( 3 )} \rightarrow 9(4) \rightarrow 12(5) \rightarrow 15(6) \rightarrow 18(7) \rightarrow 21(8) \rightarrow 24(9) \rightarrow 27(10) \rightarrow 30(11) \rightarrow 33(12) \rightarrow 36(13) \rightarrow 39(14) \rightarrow 42(15) \rightarrow 45(16$ )$\rightarrow 48(17) \rightarrow 51(18) \rightarrow 54(19) \rightarrow 57(20) \rightarrow 60(21) \rightarrow 63(22) \rightarrow 66(23) \rightarrow 69(24) \rightarrow 72(25), \ldots$

BELOW [M2]

$3(2) \rightarrow 0(1)$

\section{[M3]Series}

$\mathbf{5 ( 3 )} \rightarrow 8(4)$ $\rightarrow 11(5)$ $\rightarrow 14(6)$ $\rightarrow 17(7)$

$\rightarrow 20(8) \rightarrow 23(9) \rightarrow 26(10) \rightarrow 29(11) \rightarrow 32(12) \rightarrow 35(13) \rightarrow 38(14) \rightarrow 41(15) \rightarrow 44(16) \rightarrow 47(17) \rightarrow 50(18) \rightarrow 53(19) \rightarrow 56(20) \rightarrow 5$ $9(21) \rightarrow 62(22) \rightarrow 65(23) \rightarrow 68(24) \rightarrow 71(25), \ldots$.

BELOW [M3]

$5(3) \rightarrow 2(2)$

[M4]Series

$7(4) \rightarrow 10(5) \rightarrow 13(6) \rightarrow 16(7) \rightarrow 19(8) \rightarrow 22(9) \rightarrow 25(10) \rightarrow 28(11) \rightarrow 31(12) \rightarrow 34(13) \rightarrow 37(14) \rightarrow 40(15) \rightarrow 43(16) \rightarrow 46(17) \rightarrow$ $49(18) \rightarrow 52(19) \rightarrow 55(20) \rightarrow 58(21) \rightarrow 61(22) \rightarrow 64(23) \rightarrow 67(24) \rightarrow 70(25)$,

BELOW [M4]

$7(4) \rightarrow 4(3) \rightarrow 1(2)$

[M5]Series:

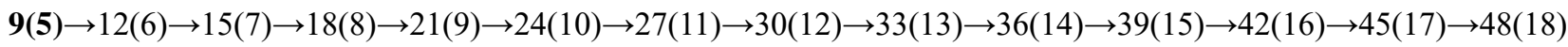
$\rightarrow 51(19) \rightarrow 54(20) \rightarrow 57(21) \rightarrow 60(22) \rightarrow 63(23) \rightarrow 66(24) \rightarrow 69(25) \rightarrow 71(26) \rightarrow 74(27) \rightarrow 77(28) \rightarrow 80(29), \ldots$

BELOW [M5]

$9(5) \rightarrow 6(4) \rightarrow 3(3) \rightarrow 0(2)$

[M6]Series:

$\mathbf{1 1}(\mathbf{6}) \rightarrow 14(7) \rightarrow 17(8) \rightarrow 20(9) \rightarrow 23(10) \rightarrow 26(11) \rightarrow 29(12) \rightarrow 32(13) \rightarrow 35(14) \rightarrow 38(15) \rightarrow 41(16) \rightarrow 44(17) \rightarrow 47(18) \rightarrow 50(19$ )$\rightarrow 53(20) \rightarrow 56(21) \rightarrow 59(22) \rightarrow 62(23) \rightarrow 65(24) \rightarrow 68(25) \rightarrow 71(26) \rightarrow 74(27) \rightarrow 77(28) \rightarrow 80(29), \ldots$

BELOW [M6]

$11(6) \rightarrow 8(5) \rightarrow 5(4) \rightarrow 2(3)$

[M7]Series:

$\mathbf{1 3}(7) \rightarrow 16(8) \rightarrow 19(9) \rightarrow 22(10) \rightarrow 25(11) \rightarrow 28(12) \rightarrow 31(13) \rightarrow 34(14) \rightarrow 37(15) \rightarrow 40(16) \rightarrow 43(17) \rightarrow 46(18) \rightarrow 49(19) \rightarrow 52(2$ $0) \rightarrow 55(21) \rightarrow 58(22) \rightarrow 61(23) \rightarrow 64(24) \rightarrow 67(25) \rightarrow 70(26) \rightarrow 73(27) \rightarrow 76(28) \rightarrow 79(29) \rightarrow 82(30), \ldots$

BELOW [M7]

$13(7) \rightarrow 10(6) \rightarrow 7(5) \rightarrow 4(4) \rightarrow 1(3)$

[M8]Series:

$\mathbf{1 5}(\mathbf{8}) \rightarrow 18(9) \rightarrow 21(10) \rightarrow 24(11) \rightarrow 27(12) \rightarrow 30(13) \rightarrow 33(14) \rightarrow 36(15) \rightarrow 39(16) \rightarrow 42(17) \rightarrow 45(18) \rightarrow 48(19) \rightarrow 51(20) \rightarrow 54($ $21) \rightarrow 57(22) \rightarrow 60(23) \rightarrow 63(24) \rightarrow 66(25) \rightarrow 69(26) \rightarrow 72(27) \rightarrow 75(28) \rightarrow 78(29) \rightarrow 81(30), .$.

BELOW [M8]

$15(8) \rightarrow 12(7) \rightarrow 9(6) \rightarrow 6(5) \rightarrow 3(4) \rightarrow 0(3)$ 
[M9]Series:

17(9) $\rightarrow 20(10) \rightarrow 23(11) \rightarrow 26(12) \rightarrow 29(13) \rightarrow 32(14) \rightarrow 35(15) \rightarrow 38(16) \rightarrow 41(17) \rightarrow 44(18) \rightarrow 47(19) \rightarrow 50(20) \rightarrow 53(21) \rightarrow 56$ $(22) \rightarrow 59(23) \rightarrow 62(24) \rightarrow 65(25) \rightarrow 68(26) \rightarrow 71(27) \rightarrow 74(28) \rightarrow 77(29) \rightarrow 80(30), \ldots$

BELOW [M9]

$17(9) \rightarrow 14(8) \rightarrow 11(7) \rightarrow 8(6) \rightarrow 5(5) \rightarrow 2(4)$

[M10]Series:

19(10) $\rightarrow 22(11) \rightarrow 25(12) \rightarrow 28(13) \rightarrow 31(14) \rightarrow 34(15) \rightarrow 37(16) \rightarrow 40(17) \rightarrow 43(18) \rightarrow 46(19) \rightarrow 49(20) \rightarrow 52(21) \rightarrow 55(22) \rightarrow 5$ $8(23) \rightarrow 61(24) \rightarrow 64(25) \rightarrow 67(26) \rightarrow 70(27) \rightarrow 73(28) \rightarrow 76(29) \rightarrow 79(30) \rightarrow 82(31), \ldots$

BELOW [M10]

$19(10) \rightarrow 16(9) \rightarrow 13(8) \rightarrow 10(7) \rightarrow 7(6) \rightarrow 4(5) \rightarrow 1(4)$

[M11]Series:

21(11) $\rightarrow 24(12) \rightarrow 27(13) \rightarrow 30(14) \rightarrow 33(15) \rightarrow 36(16) \rightarrow 39(17) \rightarrow 42(18) \rightarrow 45(19) \rightarrow 48(20) \rightarrow 51(21) \rightarrow 54(22) \rightarrow 57(23) \rightarrow 6$ $0(24), \ldots$

BELOW [M11]

$21(11) \rightarrow 18(10) \rightarrow 15(9) \rightarrow 12(8) \rightarrow 9(7) \rightarrow 6(6) \rightarrow 3(5) \rightarrow 0(4)$

[M12]Series:

$23(12) \rightarrow 26(13) \rightarrow 29(14) \rightarrow 32(15) \rightarrow 35(16) \rightarrow 38(17) \rightarrow 41(18) \rightarrow 44(19) \rightarrow 47(20) \rightarrow 50(21), \ldots$

BELOW [M12]

$23(12) \rightarrow 20(11) \rightarrow 17(10) \rightarrow 14(9) \rightarrow 11(8) \rightarrow 8(7) \rightarrow 5(6) \rightarrow 2(5)$

[M13]Series

$\mathbf{2 5}(\mathbf{1 3}) \rightarrow 28(14) \rightarrow 31(15) \rightarrow 34(16) \rightarrow 37(17) \rightarrow 40(18) \rightarrow 43(19) \rightarrow 46(20) \rightarrow 49(21) \rightarrow 52(22), \ldots$

BELOW [M13]

$25(13) \rightarrow 22(12) \rightarrow 19(11) \rightarrow 16(10) \rightarrow 13(9) \rightarrow 10(8) \rightarrow 7(7) \rightarrow 4(6) \rightarrow 1(5)$

[M-1] Series

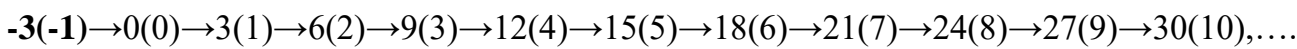

[M-2] Series

$-\mathbf{5}(-2) \rightarrow--2(-1) \rightarrow 5(0) \rightarrow 7(1) \rightarrow 10(2) \rightarrow 13(3) \rightarrow 16(4) \rightarrow 19(5) \rightarrow 21(6) \rightarrow 24(7) \rightarrow 27(8) \rightarrow 30(9) \rightarrow 33(10), \ldots$

[M-3] Series

$-7(-3) \rightarrow-4(-2) \rightarrow-1(-1) \rightarrow 2(0) \rightarrow 5(1) \rightarrow 8(2) \rightarrow 11(3) \rightarrow 14(4) \rightarrow 17(5) \rightarrow 20(6) \rightarrow 23(7) \rightarrow 26(8) \rightarrow 29(9) \rightarrow 32(10), \ldots$.

SRS-1 
$\mathrm{Au}_{4} \mathrm{~L}_{4} \mathrm{l}_{2}: \mathrm{K}=4[3.5]-4-1=9 ; \mathrm{K}(\mathrm{n})=9(4), \mathrm{S}=4 \mathrm{n}-2, \mathrm{Kp}=\mathrm{C}^{2} \mathrm{C}[\mathrm{M} 2]$

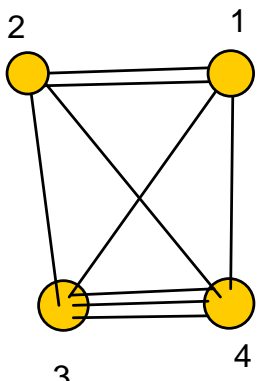

3

$\mathrm{K} 1=3.5-2=1.5$

$\mathrm{K} 2=3.5-2=1.5$

$\mathrm{K} 3=3.5-2.5=1$

$\mathrm{K} 4=3.5-2.5=1$

$\mathrm{KT}=5=4 \mathrm{~L}+2 \mathrm{I}$

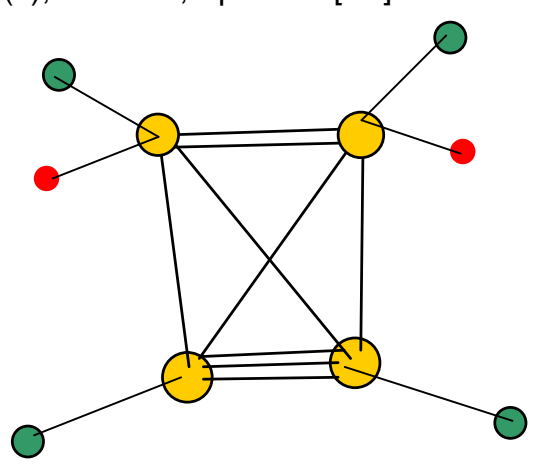

$\bigcirc=A u$

$\mathrm{O}=\mathrm{L}$

$=1$

$\mathrm{Ve}=14 n-2=14(4)-2=54 ; \mathrm{VF}=4[11]+8+2=54$

$\mathrm{Ve}=$ valence electrons, $\mathrm{VF}=$ valence electrons from cluster formula $K(n)=9(4)$ belongs to [M2]series.

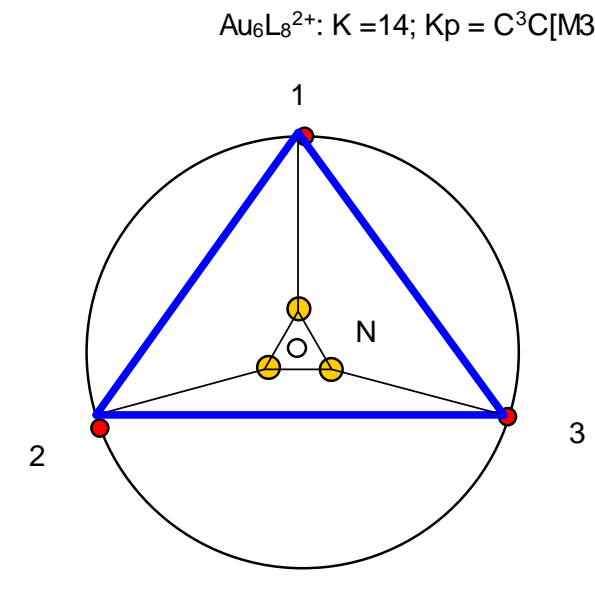

$\mathrm{KN}=2 \mathrm{n}-1=2(3)-1=5$

$\mathrm{KS}=3+3+3+\mathrm{KN}=9+5=14=\mathrm{K}$

$\mathrm{K} 1=3.5-2.5=1 ; \mathrm{K} 2=\mathrm{K} 1 ; \mathrm{K} 3=\mathrm{K} 1$

$\mathrm{Au}_{3}{ }^{2+}: \quad \mathrm{KNL}=3[3.5]+1-3(0.5)-5=5$

$\mathrm{KLT}=3+5=8=8 \mathrm{~L}$

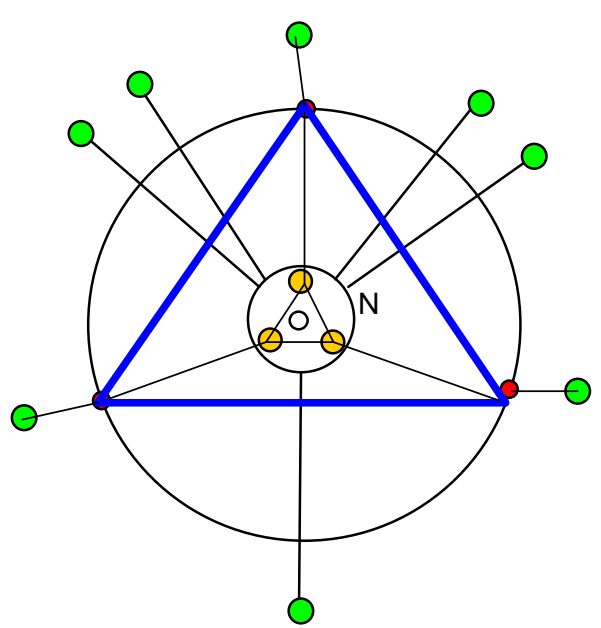

$O=L$

$$
K(n)=14(6) \text { belongs to [M3] series }
$$




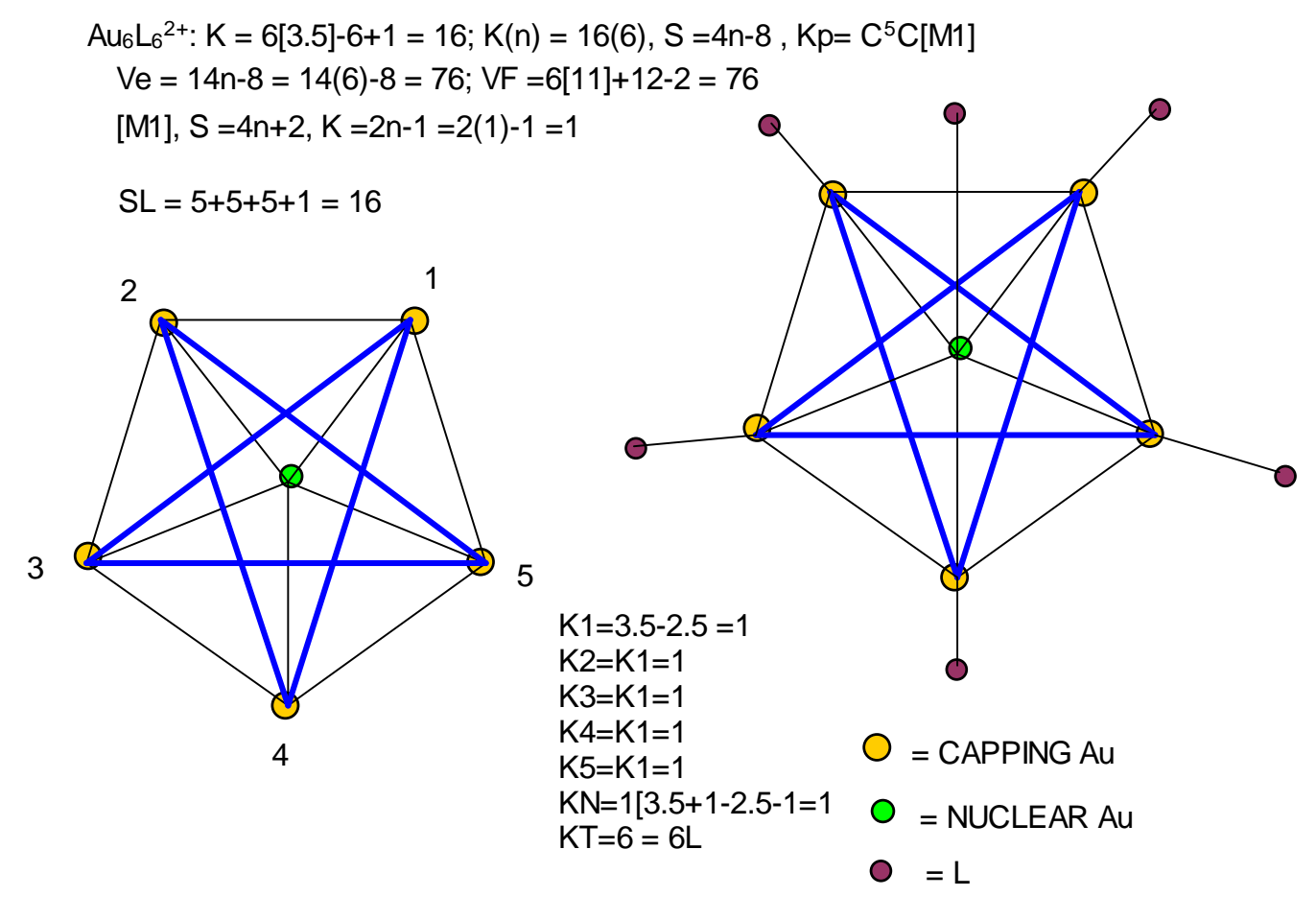

$K(n)=16(6)$ belongs to [M1] series

ISOMERIC STRUCTURE OF $\mathrm{Au}_{6} \mathrm{~L}_{6}{ }^{2+}$

$\mathrm{K}=6[3.5]-6+1=16$

$\mathrm{K}(\mathrm{n})=16(6)$

$S=4 n-8$

$\mathrm{Kp}=\mathrm{C}^{5} \mathrm{C}[\mathrm{M} 1]$

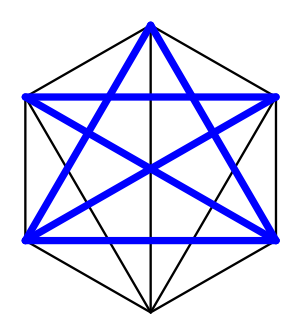



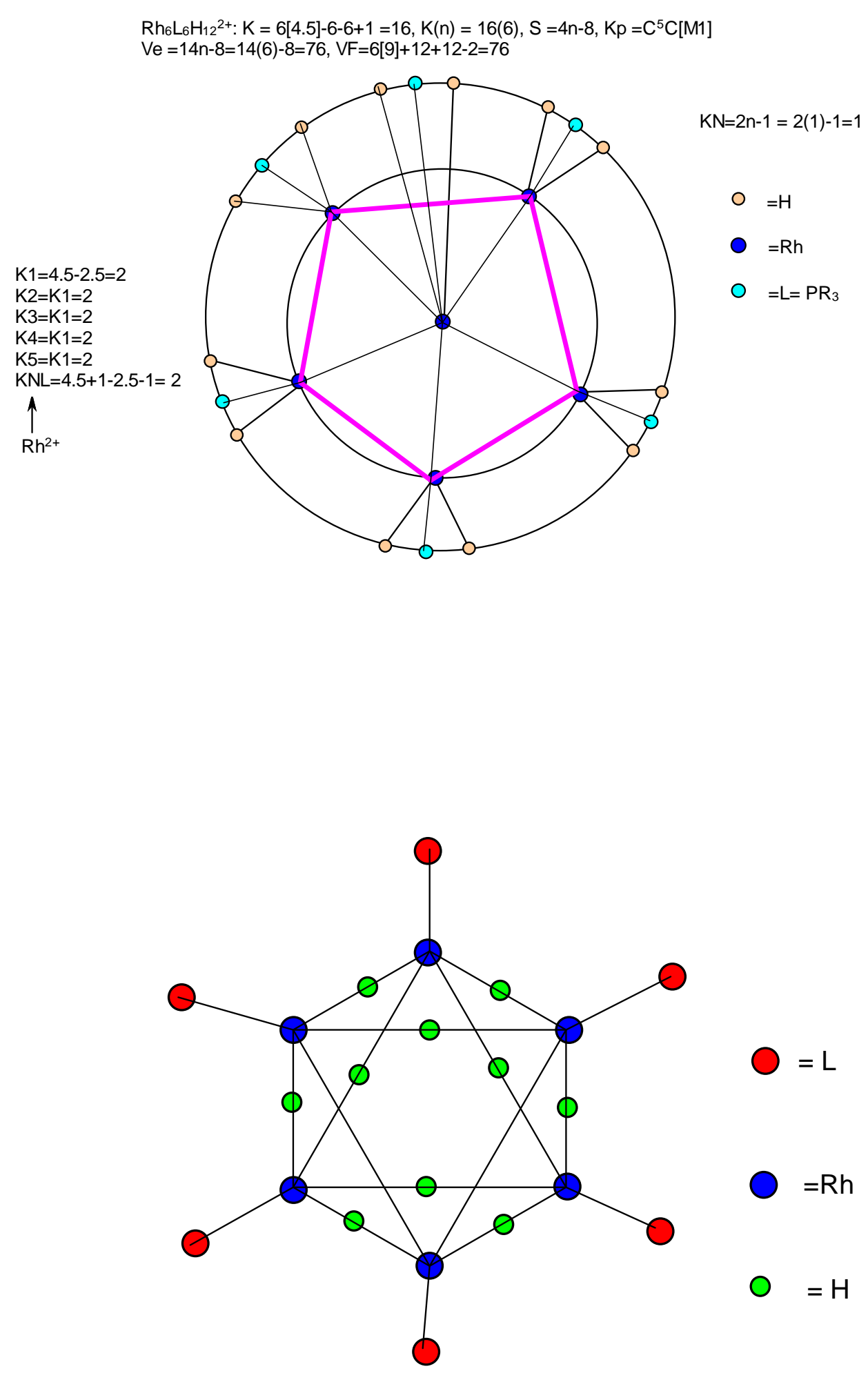

ISOMERIC SKELETAL SHAPE OF $\mathrm{Rh}_{6} \mathrm{~L}_{6} \mathrm{H}_{12}{ }^{2+}$. 


$$
\begin{aligned}
& \mathrm{K}=6[3.5]-6+1=16 \\
& \mathrm{~K}(\mathrm{n})=16(6) \\
& \mathrm{S}=4 \mathrm{n}-8 \\
& \mathrm{Kp}=\mathrm{C}^{5} \mathrm{C}[\mathrm{M} 1]
\end{aligned}
$$

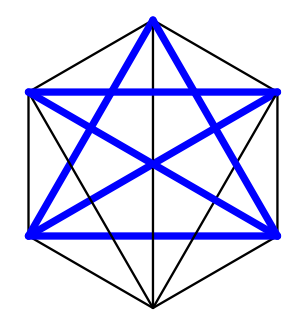

$\mathrm{Au}_{8} \mathrm{~L}_{8} \mathrm{Cl}_{2}{ }^{2+}: \mathrm{K}(\mathrm{n})=20(8), \mathrm{Kp}=\mathrm{C}^{5} \mathrm{C}[\mathrm{M} 3]$

2

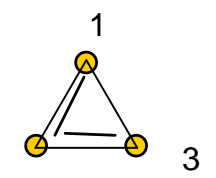

$\mathrm{K} 1=3 \cdot 5-1 \cdot 5=2$

$\mathrm{K} 2=3.5-2=1.5$ $\mathrm{K} 3=3 \cdot 5-1 \cdot 5=2$

3
2

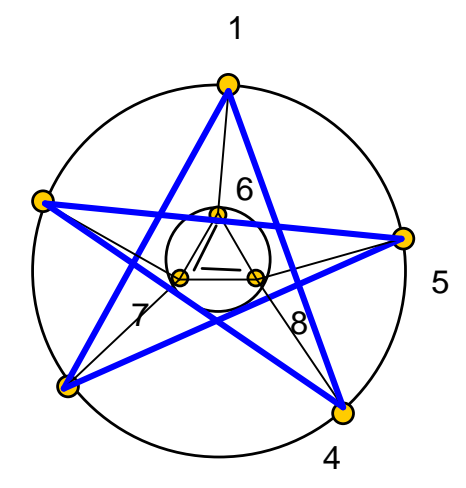

$\mathrm{O}=\mathrm{L}$

$\mathrm{K} 2=\mathrm{K} 1=1$

$\mathrm{K} 3=\mathrm{K} 1=1$

$\mathrm{K} 4=\mathrm{K} 1=1$

$\mathrm{K} 5=\mathrm{K} 1=1$

$\mathrm{K} 6=4-2=2$

$\mathrm{K} 7=3.5-3=0.5$

$\mathrm{K} 8=4-2.5=1.5$

$\mathrm{KT}=9$

$O=A u$

$\mathrm{O}=\mathrm{Cl}$

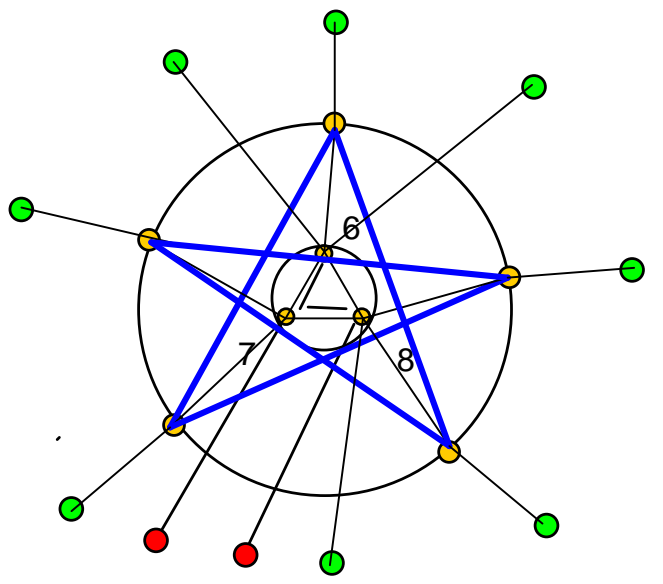

$K(n)=20(8)$ belongs to [M3] series 
$A u_{8} L_{8}{ }^{2+}: K=21(8), K p=C^{6} \mathrm{C}[\mathrm{M} 2]$

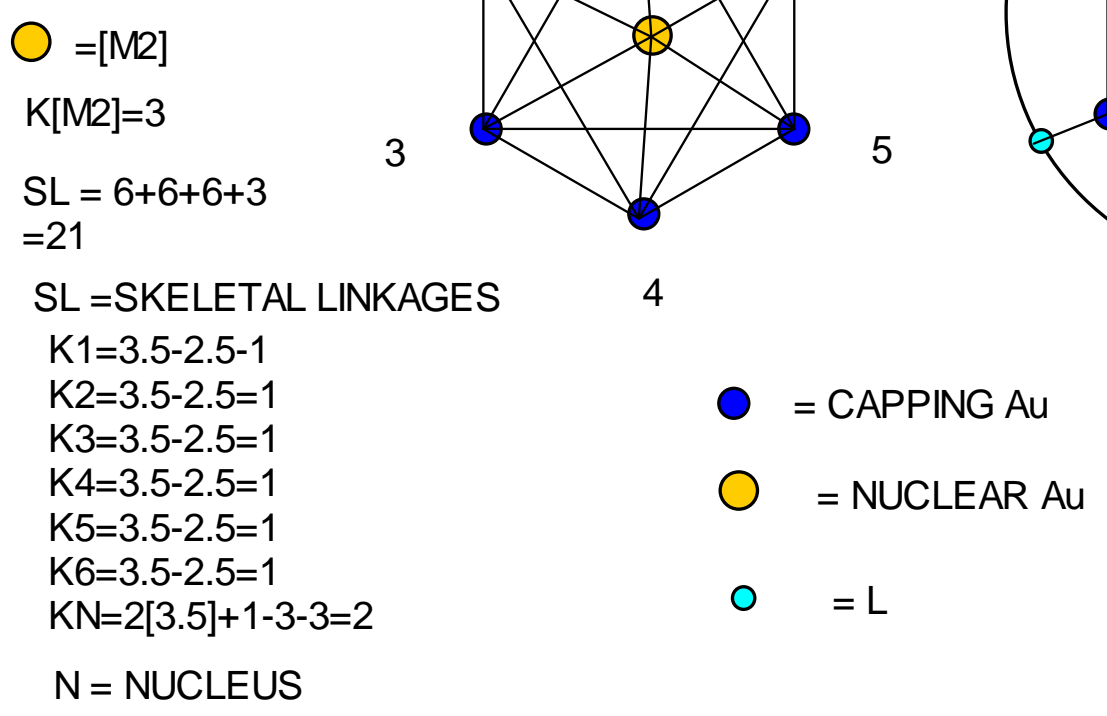

$K(n)=21(8)$ belongs to [M2] series 


$$
\mathrm{Au}_{8} \mathrm{~L}^{2+}: \mathrm{K}(\mathrm{n})=22(8), \mathrm{Kp}=\mathrm{C}^{7} \mathrm{C}[\mathrm{M} 1] \quad[\mathrm{M} 1], \mathrm{S}=4 \mathrm{n}+2, \mathrm{~K}=2 \mathrm{n}-1=2(1)-1=1
$$

$\mathrm{K} 1=3.5-2.5=1, \mathrm{~K} 2=1, \mathrm{~K} 3=1, \mathrm{~K} 4=1, \mathrm{~K} 5=1, \mathrm{~K} 6=1, \mathrm{~K} 7=1$
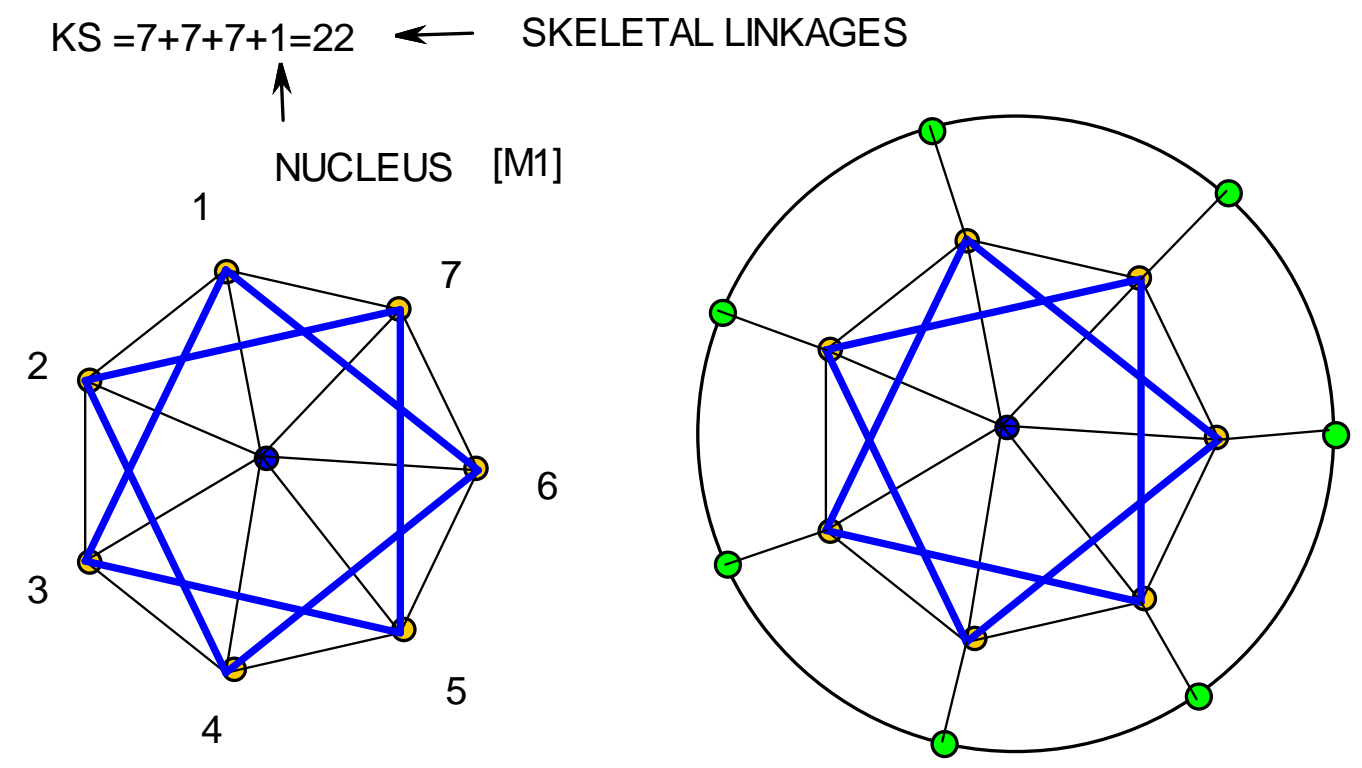

$$
\begin{aligned}
& \mathrm{KN}=1[3.5]+1-3.5-1=0 \\
& \mathrm{O}=\text { CAPPING Au }
\end{aligned}
$$$$
\mathrm{O}=\mathrm{L}
$$

$\mathrm{K}(\mathrm{n})=22(8)$ belongs to $[\mathrm{M} 1]$ series 
AugL $8^{+1}: K=9[3.5]-8+0.5=24 ; K(n)=24(9), S=4 n-12, K p=C^{7} C[M 2]$

$\mathrm{Ve}=14 \mathrm{n}-12=14(9)-12=114 ; \mathrm{VF}=9[11]+16-1=114$

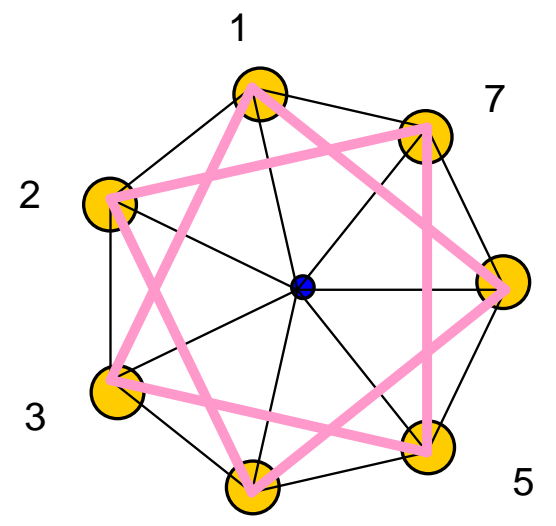

$$
\text { - }^{4}[\mathrm{M} 2] \quad \mathrm{K}[\mathrm{M} 2]=3
$$

$S L=7+7+7+3=24$

$\bigcirc=$ CAPPING Au
6

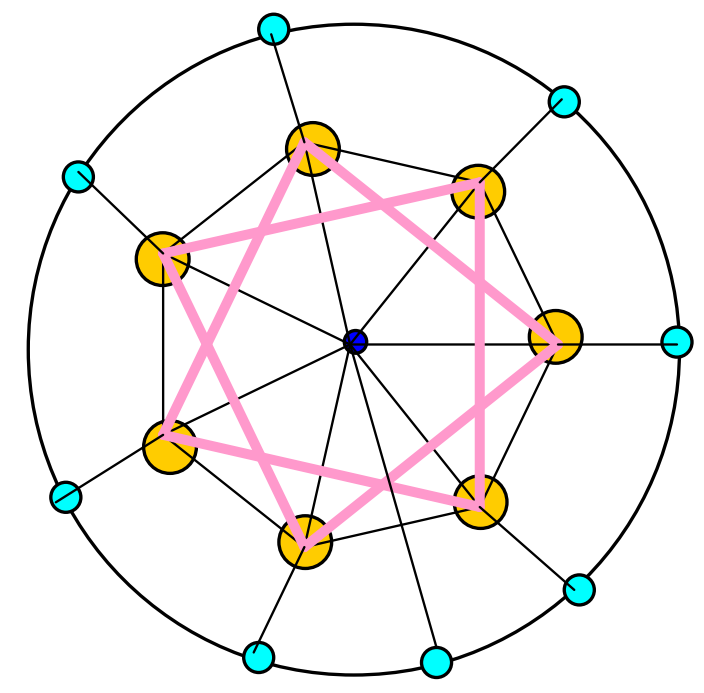

$\mathrm{K} 1=3.5-2.5=1$

$\mathrm{K} 2=\mathrm{K} 1=1$

$\mathrm{K} 3=\mathrm{K} 1=1$

$\mathrm{K} 4=\mathrm{K} 1=1$

$\mathrm{K} 5=\mathrm{K} 1=1$

$\mathrm{K} 6=\mathrm{K} 1=1$

$\mathrm{K} 7=\mathrm{K} 1=1$

$\mathrm{KN}=2[3.5]+0.5-3 \cdot 5-3=1$

$\mathrm{KT}=8$

$K(n)=24(9)$ belongs to $[M 2]$ series.

Aug $\mathrm{L}_{8}{ }^{3+}: \mathrm{K}=9[3.5]-8+1.5=25 ; \mathrm{K}(\mathrm{n})=25(9), \mathrm{S}=4 \mathrm{n}-14, \mathrm{Kp}=\mathrm{C}^{8} \mathrm{C}[\mathrm{M} 1]$

$\mathrm{Ve}=14 \mathrm{n}-14=14(9)-14=112, \mathrm{VF}=9[11]+16-3=112$

[M1], $S=4 n+2, K=2 n-1=2(1)-1=1$

$$
S L=8+8+8+1=25
$$
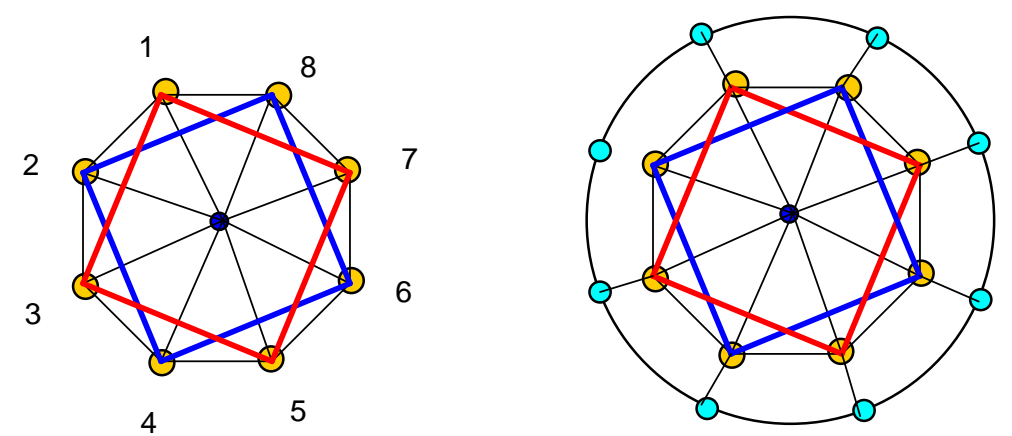

$\mathrm{K} 1=3.5-2.5=1$

$\mathrm{K} 2=\mathrm{K} 1=1$

$\mathrm{K} 3=\mathrm{K} 1=1$

$\mathrm{K} 4=\mathrm{K} 1=1$

$\mathrm{K} 5=\mathrm{K} 1=1$

$\mathrm{K} 6=\mathrm{K} 1=1$

$\mathrm{K} 7=\mathrm{K} 1=1$

$\mathrm{K} 8=\mathrm{K} 1=1$

$\mathrm{KN}=3.5+1.5-4-1=0$

- $=[\mathrm{M} 1]$

$\mathrm{O}=\mathrm{CAPPING} \mathrm{Au}$

$\mathrm{O}=\mathrm{L}$

$K(n)=25(9)$ belongs to $[M 1]$ series 
$\mathrm{Au}_{10} \mathrm{~L}_{8} \mathrm{Cl}^{+1}: \mathrm{K}=10[3.5]-8-0.5+0.5=27 ; \mathrm{K}(\mathrm{n})=27(10), \mathrm{S}=4 \mathrm{n}-14, \mathrm{Kp}=\mathrm{C}^{8} \mathrm{C}[\mathrm{M} 2] \quad[\mathrm{M} 2], \mathrm{S}=4 \mathrm{n}+2, \mathrm{~K}=2 \mathrm{n}-1$ $=2(2)-1=3$

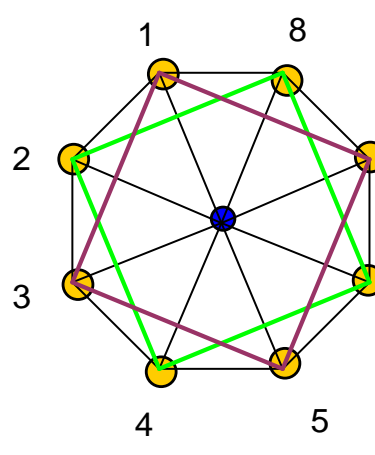

\section{7}

6

$\mathrm{K} 1=3 \cdot 5-2 \cdot 5=1$

$\mathrm{K} 2=\mathrm{K} 1=1$

$\mathrm{K} 3=\mathrm{K} 1=1$

$\mathrm{K} 4=\mathrm{K} 1=1$

$\mathrm{K} 5=\mathrm{K} 1=1$

$\mathrm{K} 6=\mathrm{K} 1=1$

$\mathrm{K} 7=\mathrm{K} 1=1$

$\mathrm{K} 8=\mathrm{K} 1=1$

$\mathrm{KN}=2[3.5]+0.5-4-3=0.5$

$\mathrm{KT}=8.5$

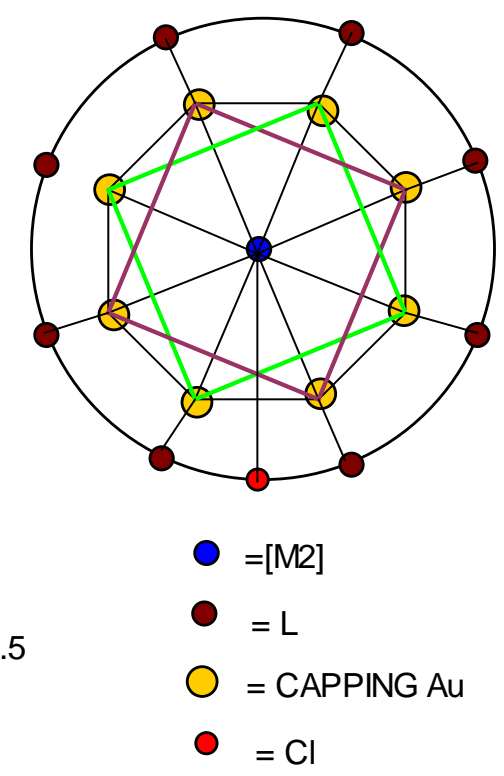

$K(n)=27(10)$ belongs to [M2] series. 
$\mathrm{Au}_{10} \mathrm{~L}_{6} \mathrm{Cl}_{3}{ }^{+1}: \mathrm{K}=10[3.5]-6-1.5+0.5=28(10), \mathrm{S}=4 \mathrm{n}-16, \mathrm{Kp}=\mathrm{C}^{9} \mathrm{C}[\mathrm{M} 1] \quad$ [M1], $\mathrm{K}=1$

$\mathrm{Ve}=14 \mathrm{n}-16=14(10)-16=124 ; \mathrm{VF}=10[11]+12+3-1=124$

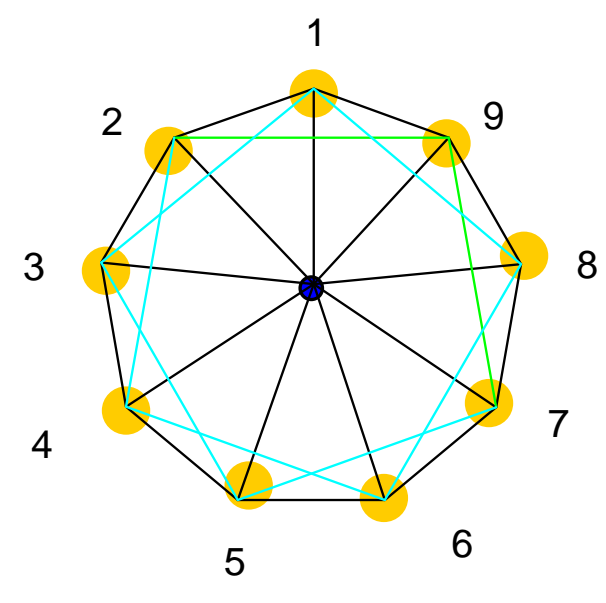

$\mathrm{SL}=9+9+9+1=28$

$=[\mathrm{M} 1]$

$=$ CAPPING Au

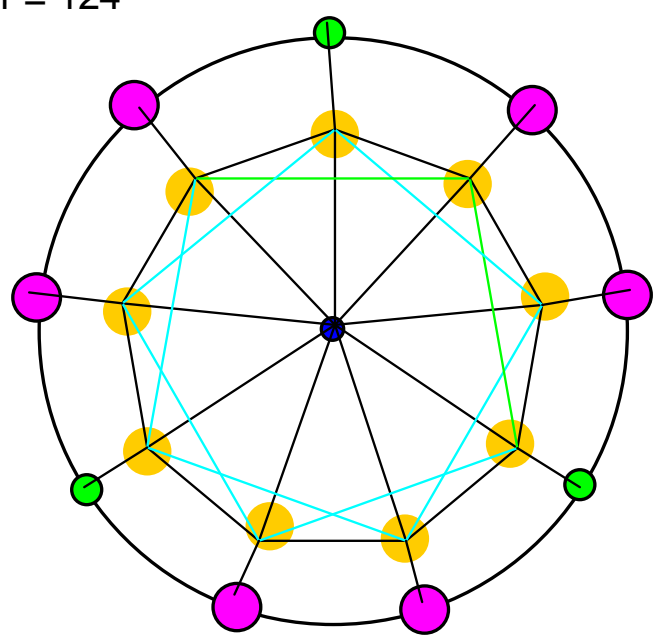

$\mathrm{K} 1=3.5-2.5=1$

$\mathrm{K} 2=\mathrm{K} 1=1$

$\mathrm{K} 3=\mathrm{K} 1=1$

$\mathrm{K} 4=\mathrm{K} 1=1$

$\mathrm{K} 5=\mathrm{K} 1=1$

$\mathrm{K} 6=\mathrm{K} 1=1$

$\mathrm{K} 7=\mathrm{K} 1=1$

$\mathrm{K} 8=\mathrm{K} 1=1$

$\mathrm{K} 9=\mathrm{K} 1=1$

$\mathrm{K} 10=\mathrm{K} 1=1$

$\mathrm{KN}=3.5+0.5--4.5-1=-1.5$

$\mathrm{KT}=9-1.5=7.5=6 \mathrm{~L}+3 \mathrm{Cl}$

BLACKHOLE NUCLEUS

$K(n)=28(10)$ belongs to $[M 1]$ series. 
$\mathrm{Au}_{10} \mathrm{~L}_{3} \mathrm{R}_{4}: \mathrm{K}=10[3.5]-3-2=30(10), \mathrm{S}=4 \mathrm{n}-20, \mathrm{Kp}=\mathrm{C}^{11} \mathrm{C}[\mathrm{M}-1]$

$\mathrm{Ve}=14 \mathrm{n}-20=14(10)-20=120, \mathrm{VF}=10[11]+6+4=120$

$[M-1], S=4 n+2, K=2 n-1=2(-1)-1=-3$

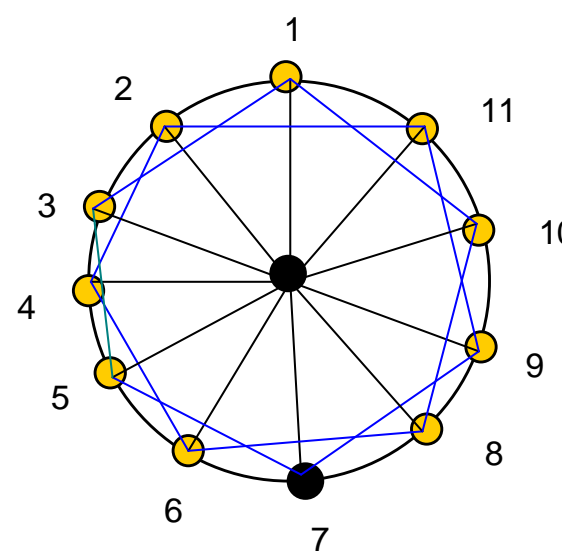

$\mathrm{K} 1=3.5-2 \cdot 5=1$

$\mathrm{K} 2=\mathrm{K} 1=1$

$\mathrm{K} 3=\mathrm{K} 1=1$

$\mathrm{K} 4=\mathrm{K} 1=1$

$\mathrm{K} 5=\mathrm{K} 1=1$

$10 \mathrm{~K} 6=\mathrm{K} 1=1$

$\mathrm{K} 7=\mathrm{K} 1=1$

$\mathrm{K} 8=\mathrm{K} 1=1$

$\mathrm{K} 9=\mathrm{K} 1=1$

$\mathrm{K} 10=\mathrm{K} 1=1$

$\mathrm{K} 11=\mathrm{K} 1=1$

$\mathrm{KN}=-[3.5]-5.5-(-3)=-6$

$\mathrm{KT}=11-6=5=3 \mathrm{~L}+4 \mathrm{R}$

BLACK HOLE NUCLEUS

$\mathrm{SL}=11+11+11+[\mathrm{M}-1]$

$=33+(-3)=30$

$=$ BLACK HOLE NUCLES/SKELETAL ELEMENT

$\bigcirc=$ CAPPING Au

$K(n)=30(10)$ belongs to [M-1] series.

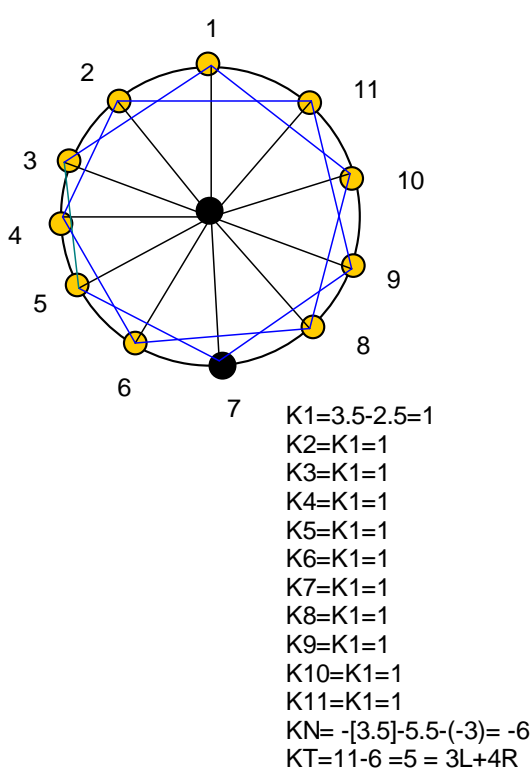

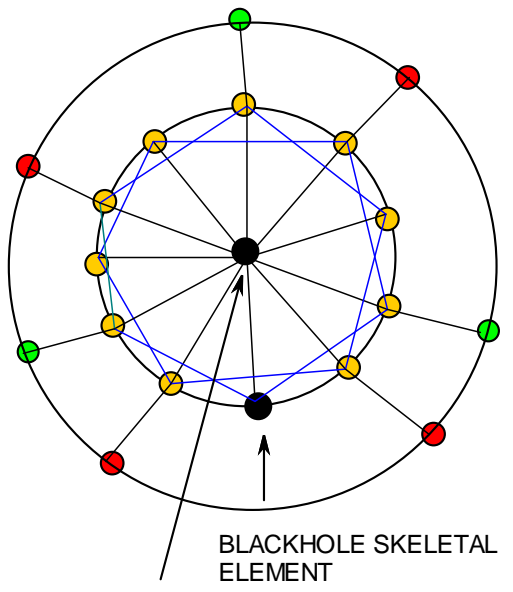

BLACKHOLE NUCLEUS

$\mathrm{O}=\mathrm{R}$

O $=\mathrm{L}$ 
$\mathrm{Au}_{11} \mathrm{~L}_{7} \mathrm{X}_{3}: \mathrm{K}=11[3.5]-7-1.5=30, \mathrm{~K}(\mathrm{n})=30(11), \mathrm{S}=4 \mathrm{n}-16, \mathrm{Kp}=\mathrm{C}^{9} \mathrm{C}[\mathrm{M} 2]$ $\mathrm{Ve}=14 \mathrm{n}-16=14(11)-16=138, \mathrm{VF}=11[11]+14+3=138 ; \mathrm{K}[\mathrm{M} 2]=3$
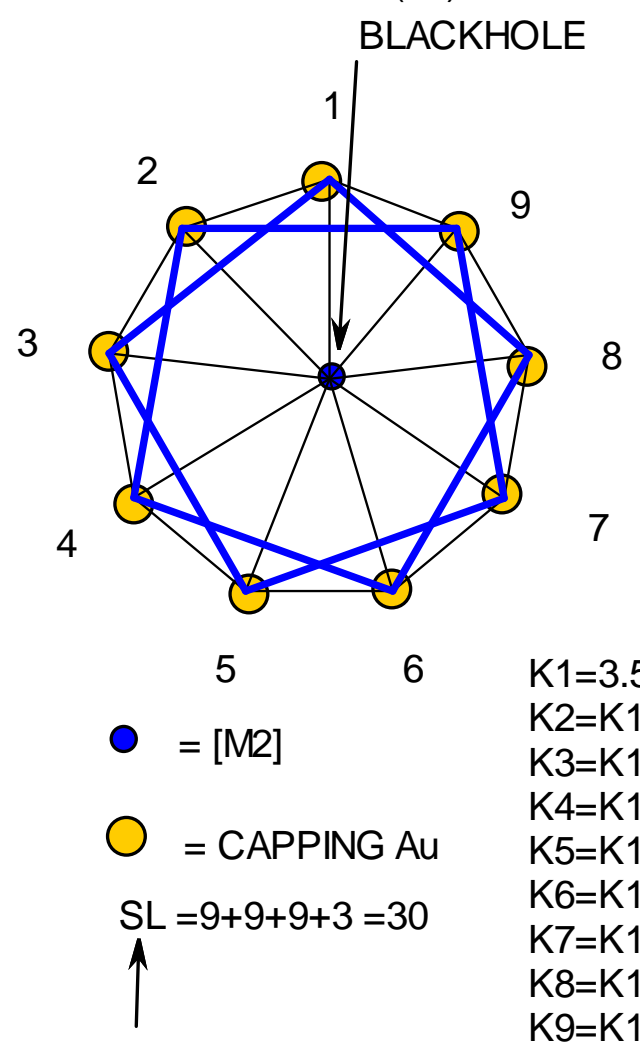

$\mathrm{K} 1=3.5-2.5=1$

$\mathrm{K} 2=\mathrm{K} 1=1$

$\mathrm{K} 3=\mathrm{K} 1=1$

$\mathrm{K} 4=\mathrm{K} 1=1$

$\mathrm{K} 5=\mathrm{K} 1=1$

$\mathrm{K} 6=\mathrm{K} 1=1$

$\mathrm{K} 7=\mathrm{K} 1=1$

$\mathrm{K} 8=\mathrm{K} 1=1$

$\mathrm{K} 9=\mathrm{K} 1=1$

SKELETAL LINKAGES

$\mathrm{KN}=2[3.5]-4.5-3=-0.5$

$\mathrm{KT}=9+(-.5)=8.5=7 \mathrm{~L}+3 \mathrm{X}$

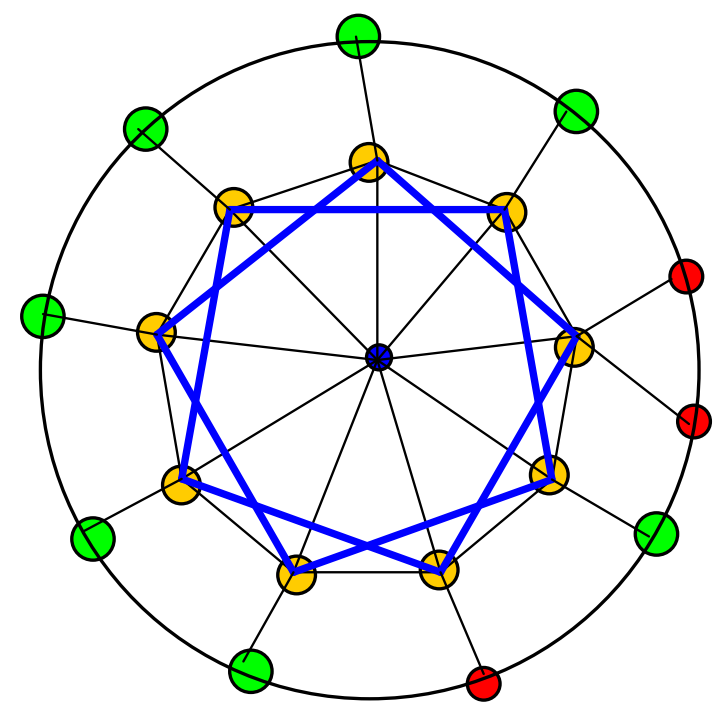

$K(n)=30(11)$ belongs to [M2] series. 
$\mathrm{Au}_{11} \mathrm{~L}_{7} \mathrm{X}_{3}: \mathrm{K}=11[3.5]-7-1.5=30, \mathrm{~K}(\mathrm{n})=30(11), \mathrm{S}=4 \mathrm{n}-16, \mathrm{Kp}=\mathrm{C}^{9} \mathrm{C}[\mathrm{M} 2]$ $\mathrm{Ve}=14 \mathrm{n}-16=14(11)-16=138, \mathrm{VF}=11[11]+14+3=138 ; \mathrm{K}[\mathrm{M} 2]=3$
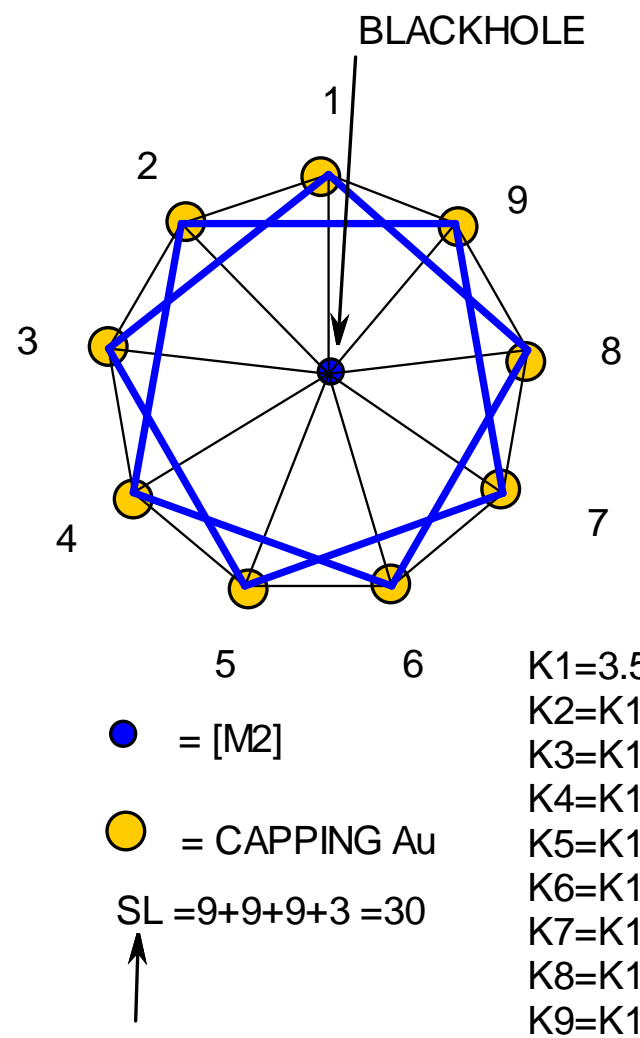

SKELETAL LINKAGES

$\mathrm{K} 1=3.5-2.5=1$

$\mathrm{K} 2=\mathrm{K} 1=1$

$\mathrm{K} 3=\mathrm{K} 1=1$

$\mathrm{K} 4=\mathrm{K} 1=1$

$\mathrm{K} 5=\mathrm{K} 1=1$

$\mathrm{K} 6=\mathrm{K} 1=1$

$\mathrm{K} 7=\mathrm{K} 1=1$

$\mathrm{K} 8=\mathrm{K} 1=1$

$\mathrm{K} 9=\mathrm{K} 1=1$

$\mathrm{KN}=2[3.5]-4.5-3=-0.5$

$\mathrm{KT}=9+(-.5)=8.5=7 \mathrm{~L}+3 \mathrm{X}$

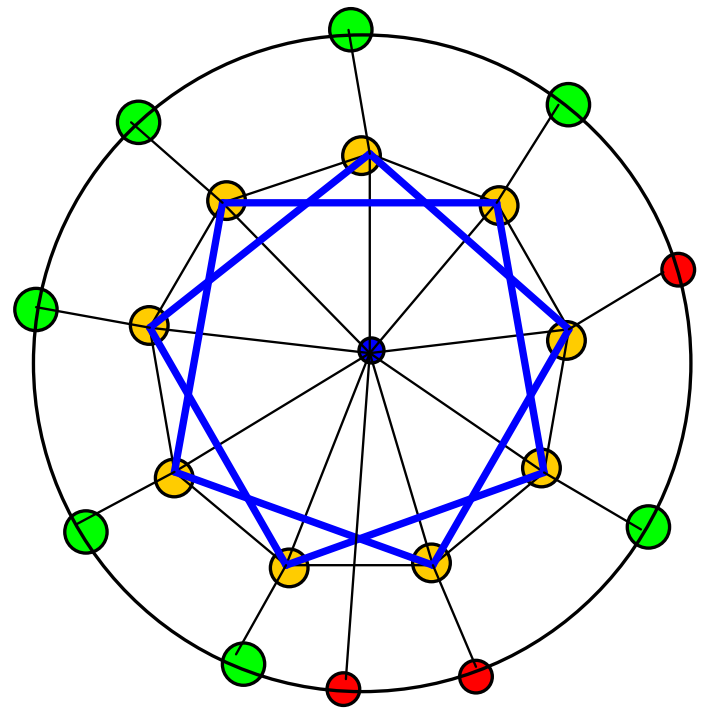

$\mathrm{O}=\mathrm{Cl}$

$\bigcirc=L$

\section{ISOMERISM}




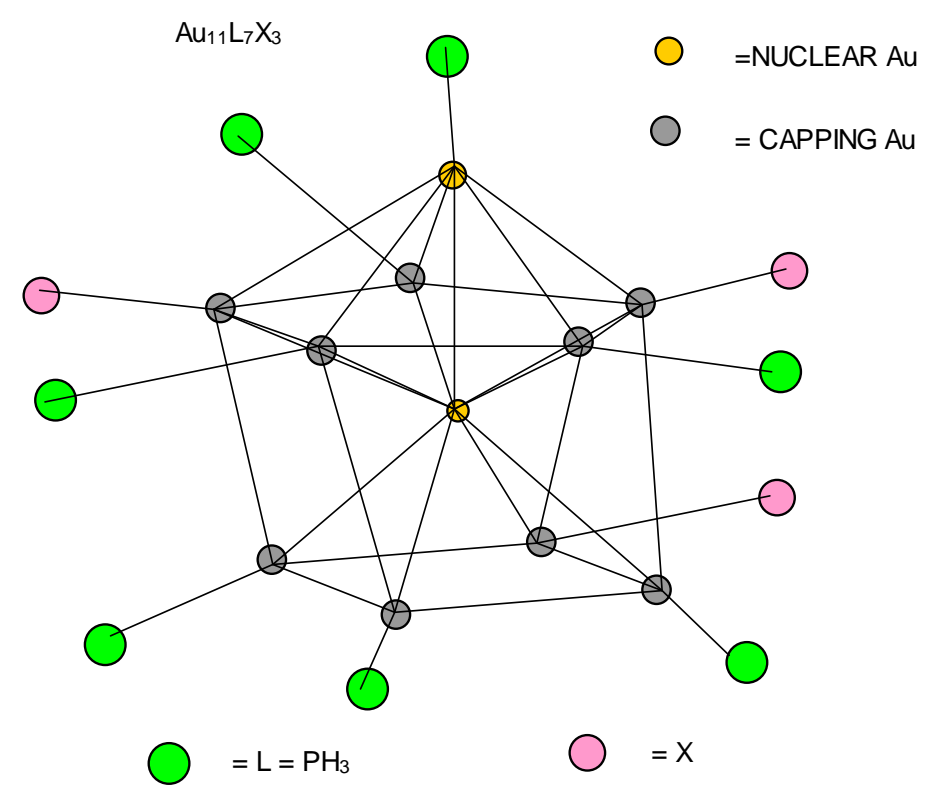

SKETCH OF THE OBSERVED STRUCTURE

$\mathrm{Au}_{11} \mathrm{~L}_{10^{3+}}: \mathrm{K}=11[3.5]-10+1.5=30 ; \mathrm{K}(\mathrm{n})=30(11), \mathrm{S}=4 \mathrm{n}-16, \mathrm{Kp}=\mathrm{C}^{9} \mathrm{C}[\mathrm{M} 2]$ $\mathrm{Ve}=14 n-16=14(11)-16=138 ; \mathrm{VF}=11[11]+20-3=138$

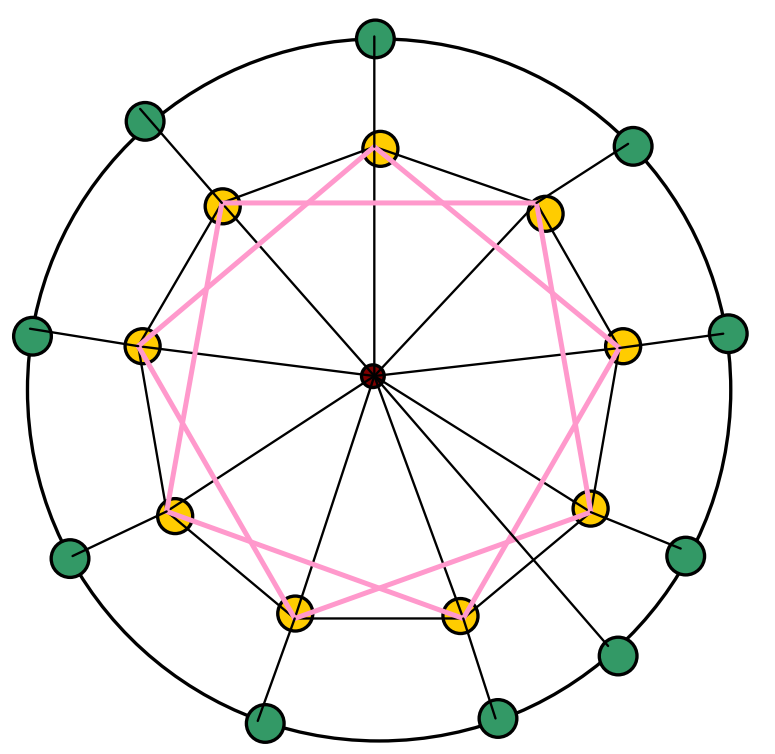

$\mathrm{SL}=9+9+9+3=30$

$\mathrm{SL}=$ SKELETAL LINKAGES

PERIPHERY, $\mathrm{K}=1$

$\mathrm{KT}=9$

$\mathrm{KN}=2[3.5]+1.5-4.5-3=1$

GRD TOTAL $=9+1=10$

TOTAL K VALUES $=\mathrm{KT}$ 
$\mathrm{Au}_{12} \mathrm{~L}_{10} \mathrm{Cl}^{3+}: \mathrm{K}=12[3.5]-10-0.5+1.5=33 ; \mathrm{K}(\mathrm{n})=33(12), \mathrm{S}=4 \mathrm{n}-18, \mathrm{Kp}=\mathrm{C}{ }^{10} \mathrm{C}[\mathrm{M} 2]$

$\mathrm{Ve}=14 \mathrm{n}-18=14(12)-18=150 ; \mathrm{VF}=12[11]+20+1-3=150$

[M2], $\mathrm{K}=3$

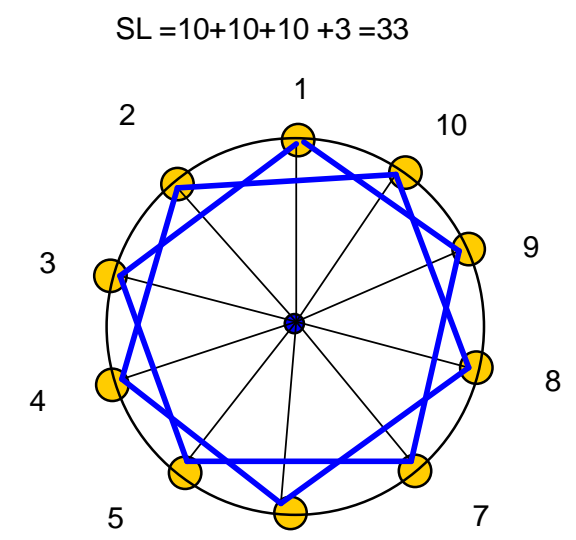

PERIPHERY K =3.5-2.5 = 1 FOR EVERY ELEMENT

$\mathrm{KN}=2[3.5]+1.5--5-3=0.5$

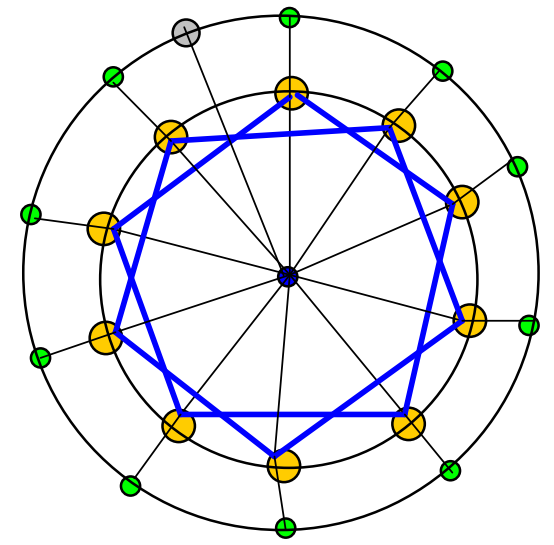

- $=[\mathrm{M} 2]$

$=$ CAPPING Au

$\mathrm{O}=\mathrm{L}$

$\mathrm{K}(\mathrm{n})=33(12)$ belongs to $[\mathrm{M} 2]$ series

$\mathrm{Au}_{13} \mathrm{~L}_{10} \mathrm{Cl}_{2}{ }^{3+}: \mathrm{K}=13[3.5]-10-1+1.5=36 ; \mathrm{K}(\mathrm{n})=36(13), \mathrm{S}=4 \mathrm{n}-20, \mathrm{Kp}=\mathrm{C}^{11} \mathrm{C}[\mathrm{M} 2]$

$\mathrm{Ve}=14 \mathrm{n} \_20=14(13)-20=162$

$\mathrm{VF}=13[11]+20+2-3=$

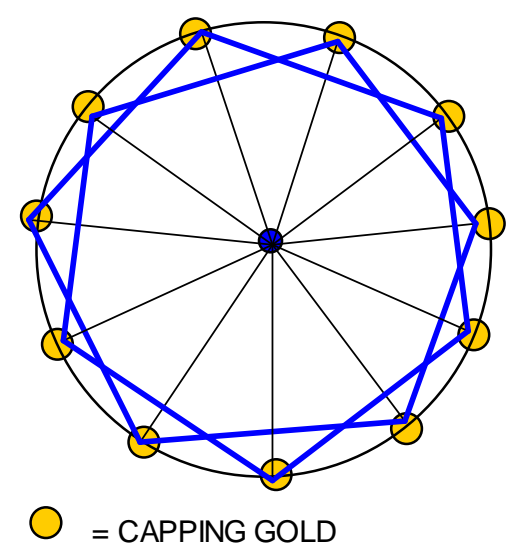

$\mathrm{K}=1$ FOR EVERY PERIPHERY

SKELETAL ELEMENT, TOTAL $=11=10 \mathrm{~L}+2 \mathrm{Cl}$

$\mathrm{KN}=2[3.5]+1.5-5.5-3=0$

$\mathrm{SL}=11+11+11+3=36$

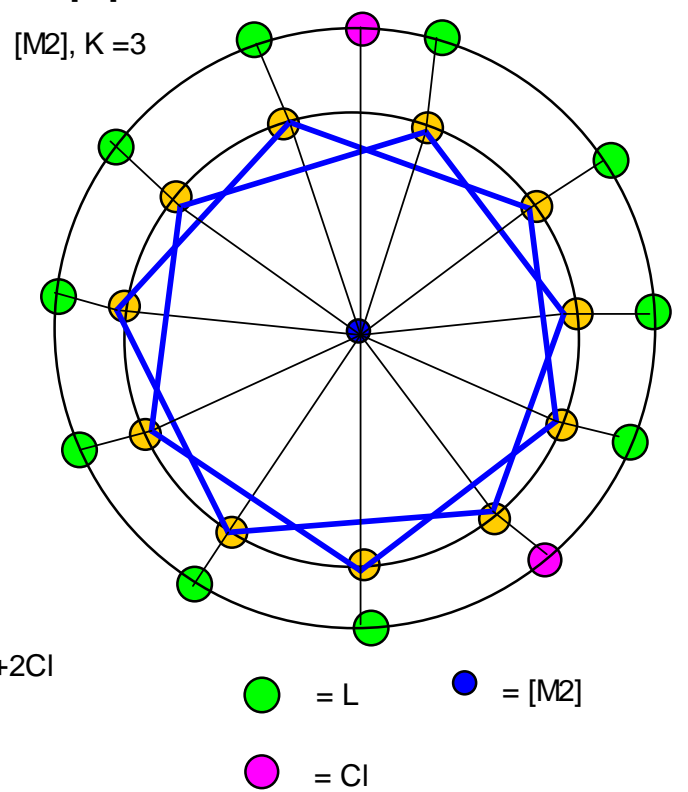

ISOMERISM

$$
K(n)=36(13) \text { belongs to [M2] series. }
$$


$\mathrm{Au}_{13} \mathrm{~L}_{10} \mathrm{Cl}_{2}{ }^{3+}: \mathrm{K}=13[3.5]-10-1+1.5=36 ; \mathrm{K}(\mathrm{n})=36(13), \mathrm{S}=4 \mathrm{n}-20, \mathrm{Kp}=\mathrm{C}^{11} \mathrm{C}[\mathrm{M} 2]$ $\mathrm{Ve}=14 \mathrm{n} \_20=14(13)-20=162 \quad \mathrm{VF}=13[11]+20+2-3=$

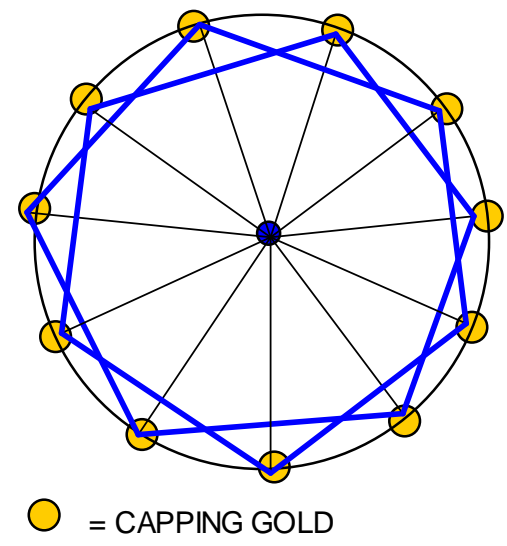

$\mathrm{K}=1$ FOR EVERY PERIPHERY

SKELETAL ELEMENT, TOTAL $=11=10 \mathrm{~L}+2 \mathrm{Cl}$

$\mathrm{KN}=2[3.5]+1.5-5 \cdot 5-3=0$

$\mathrm{SL}=11+11+11+3=36$

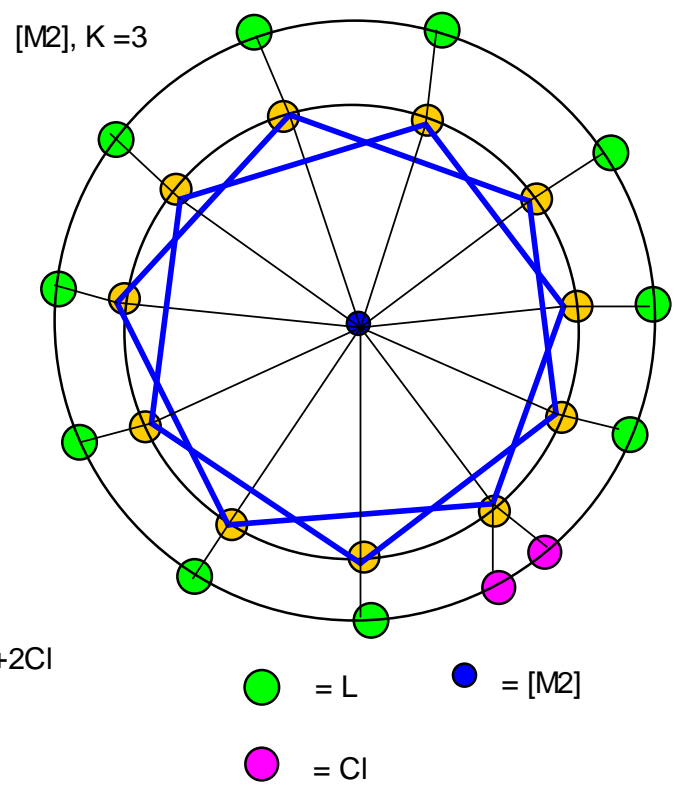

ISOMERISM

$\mathrm{Au}_{15} \mathrm{~L}_{12}(\mathrm{Cl})(\mathrm{H})^{3+}: \mathrm{K}=15[3.5]-12--1+1.5=41 ; \mathrm{K}(\mathrm{n})=41(15), \mathrm{S}=4 \mathrm{n}-22, \mathrm{Kp}=\mathrm{C}^{12} \mathrm{C}[\mathrm{M} 3]$

$\mathrm{Ve}=14 \mathrm{n}-22=14(15)-22=188 ; \mathrm{VF}=15[11]+24+2-3=188$

[M3], $K=2 n-1=2(3)-1=5$

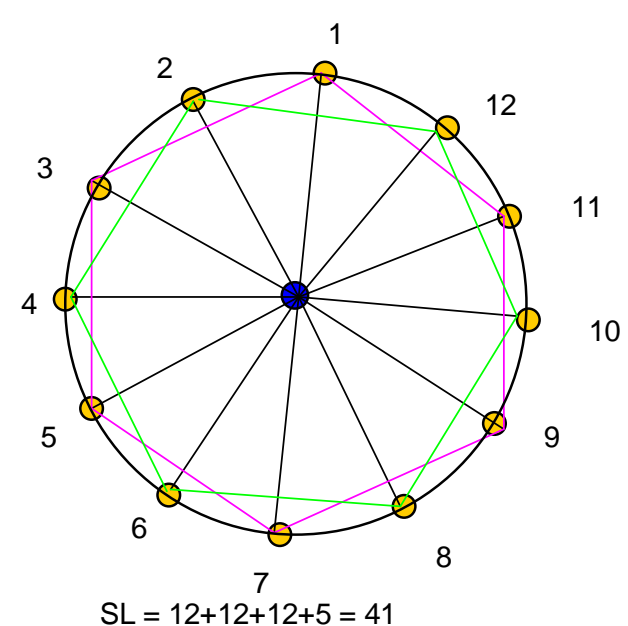

$S L=12+12+12+5=41$
$\mathrm{K} 1=3.5-2 \cdot 5=1$

$\mathrm{K} 2=1$

$\mathrm{K} 3=1$

$\mathrm{K} 4=1$

$\mathrm{K} 5=1$

$\mathrm{K} 6=1$

$\mathrm{K} 7=1$

$\mathrm{K} 8=1$

$\mathrm{K} 9=1$

$\mathrm{K} 10=1$

$\mathrm{K} 11=1$

$\mathrm{K} 12=1$

$\mathrm{KN}=3[3.5]+1.5--6-5=1$

$\mathrm{KT}=13=12 \mathrm{~L}+1 \mathrm{Cl}+1 \mathrm{H}$

$=[\mathrm{M} 3]$

$0=$ CAPPING Au

$K(n)=41(15)$ belongs to [M3] series. 


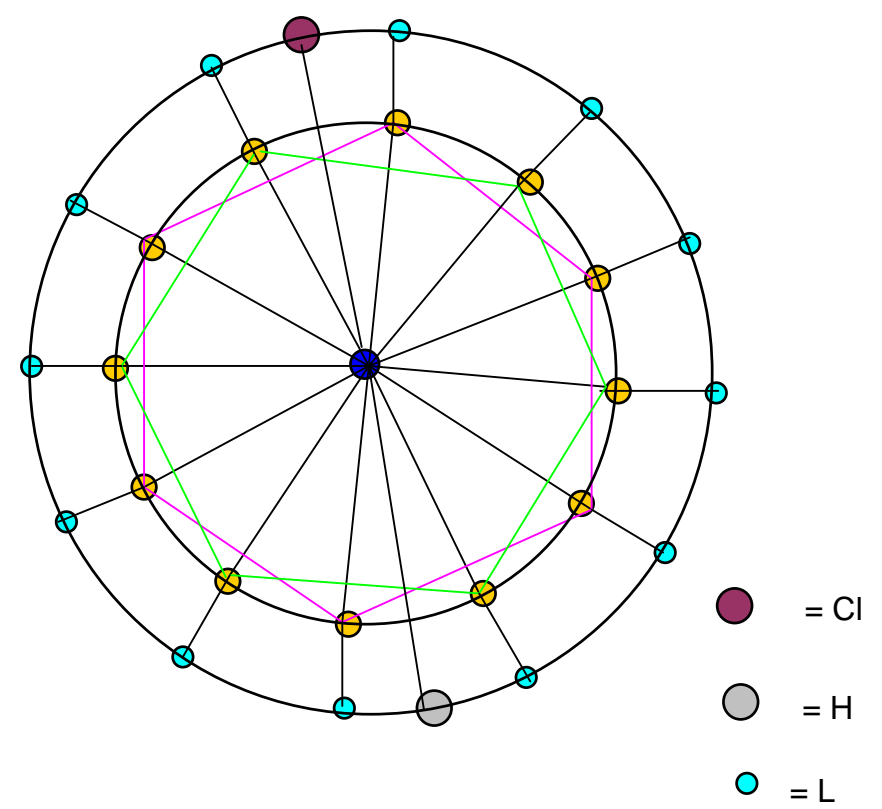

$\mathrm{K} 1=3 \cdot 5-2 \cdot 5=1$

$\mathrm{K} 2=1$

$\mathrm{K} 3=1$

$\mathrm{K} 4=1$

$\mathrm{K} 5=1$

$\mathrm{K} 6=1$

$\mathrm{K} 7=1$

$\mathrm{K} 8=1$

$\mathrm{K} 9=1$

$\mathrm{K} 10=1$

$\mathrm{K} 11=1$

$\mathrm{K} 12=1$

$\mathrm{KN}=3[3.5]+1.5--6-5=1$

$\mathrm{KT}=13=12 \mathrm{~L}+1 \mathrm{Cl}+1 \mathrm{H}$

$\mathrm{Au}_{14} \mathrm{~L}_{8} \mathrm{R}_{4}: \mathrm{K}=14[3.5]-8-2=39, \mathrm{~K}(\mathrm{n})=39(14), \mathrm{S}=4 \mathrm{n}-22, \mathrm{Kp}=\mathrm{C}^{12} \mathrm{C}[\mathrm{M} 2]$

$\mathrm{Ve}=14 \mathrm{n}-22=14(14)-22=174 ; \mathrm{VF}=14[11]+16+4=174$

[M2], $\mathrm{K}=3$

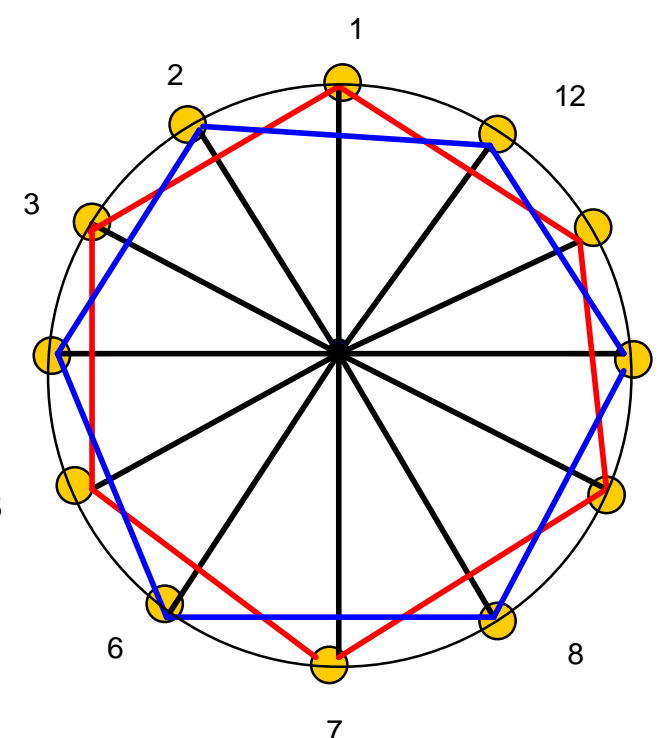

$\mathrm{K} 1=1$

$\mathrm{K} 2=1$

$\mathrm{K} 3=1$

$\mathrm{K} 4=1$

$11 \quad \mathrm{~K} 5=1$

$\mathrm{K} 6=1$

$\mathrm{K} 7=1$

$\mathrm{K} 8=1$

$10 \mathrm{~K} 10=1$

$\mathrm{K} 11=1$

$\mathrm{K} 12=1$

$\mathrm{KN}=2[3.5]-6-3=-2$

9

$\mathrm{KT}=12+(-2)=10=8 \mathrm{~L}+4 \mathrm{R}$

- $=[\mathrm{M} 2]$

$==$ CAPPING Au

$\mathrm{SL}=12+12+12+3=39$

$K(n)=39(14)$ belongs to [M2] series. 
$\mathrm{Au}_{14} \mathrm{~L}_{8} \mathrm{R}_{4}: \mathrm{K}=14[3.5]-8-2=39, \mathrm{~K}(\mathrm{n})=39(14), \mathrm{S}=4 \mathrm{n}-22, \mathrm{Kp}=\mathrm{C}^{12} \mathrm{C}[\mathrm{M} 2]$

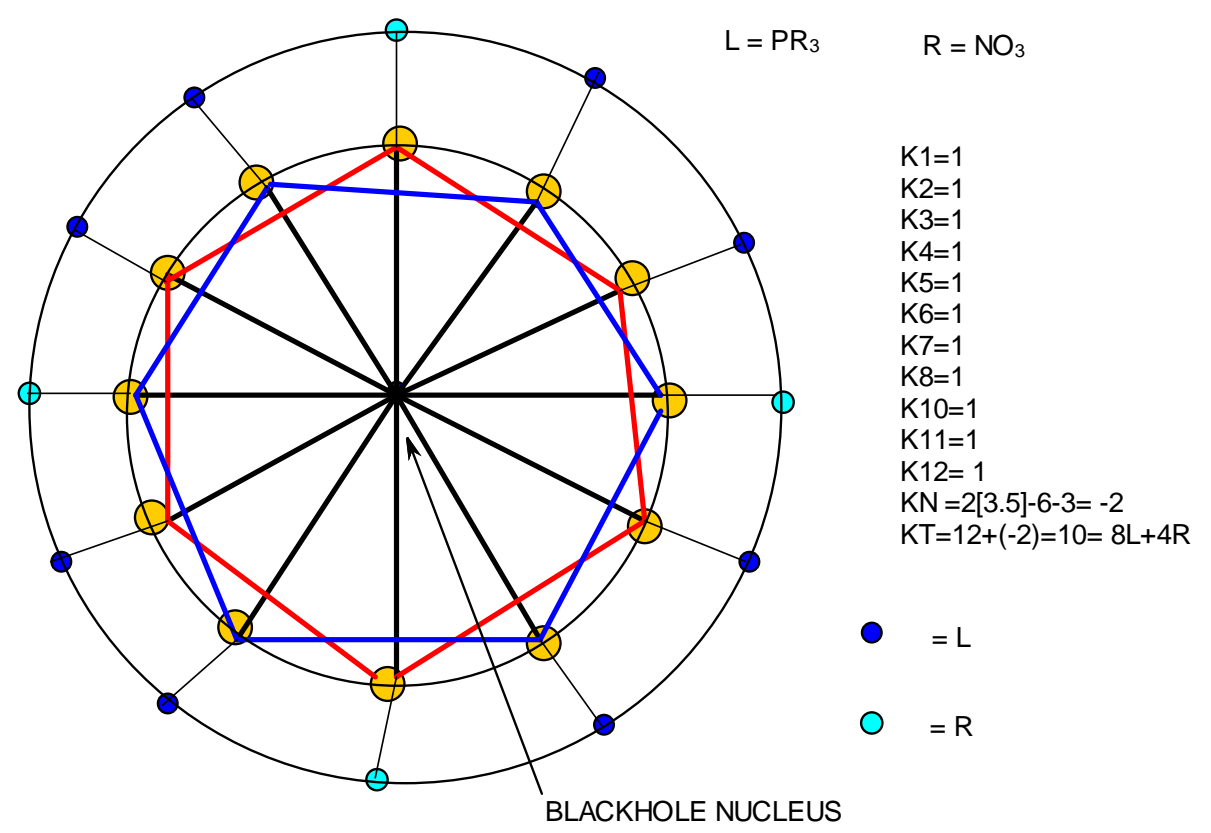

$K(n)=59(20)$ belongs to [M0] series.

$\mathrm{Au}_{20} \mathrm{~L}_{10} \mathrm{Cl}_{4}{ }^{2+}: \mathrm{K}=20[3.5]-10-2+1=59, \mathrm{~K}(\mathrm{n})=59(20), \mathrm{S}=4 \mathrm{n}-38, \mathrm{Kp}=\mathrm{C}^{20} \mathrm{C}[\mathrm{MO}]$

$\mathrm{Ve}=14 \mathrm{n}-38=14(20)-38=242, \mathrm{VF}=20[11]+20+4-2=242$

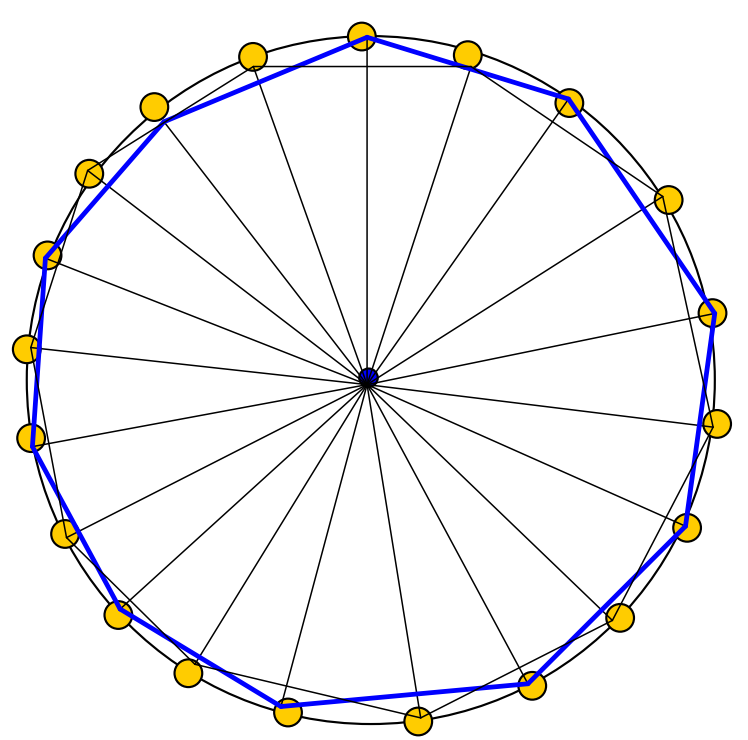

$S L=20+20+20+[M 0]$

[MO], $\mathrm{K}=2 \mathrm{n}-1=2(0)-1=-1$

$\mathrm{KT}=60-1=59$

$\mathrm{PL}=20[3.5]-20(2.5)=20$

$\mathrm{KNL}=0(3.5)+1-20(0.5)-[-1]$

$=1-10+1=-8$

$\mathrm{NET}=20-8=12=10 \mathrm{~L}+4 \mathrm{Cl}$ 
$\mathrm{Au}_{20} \mathrm{~L}_{10} \mathrm{Cl}_{4}{ }^{2+}: \mathrm{K}=20[3.5]-10-2+1=59, \mathrm{~K}(\mathrm{n})=59(20), \mathrm{S}=4 \mathrm{n}-38, \mathrm{Kp}=\mathrm{C}^{20} \mathrm{C}[\mathrm{MO}]$

$\mathrm{Ve}=14 \mathrm{n}-38=14(20)-38=242, \mathrm{VF}=20[11]+20+4-2=242$

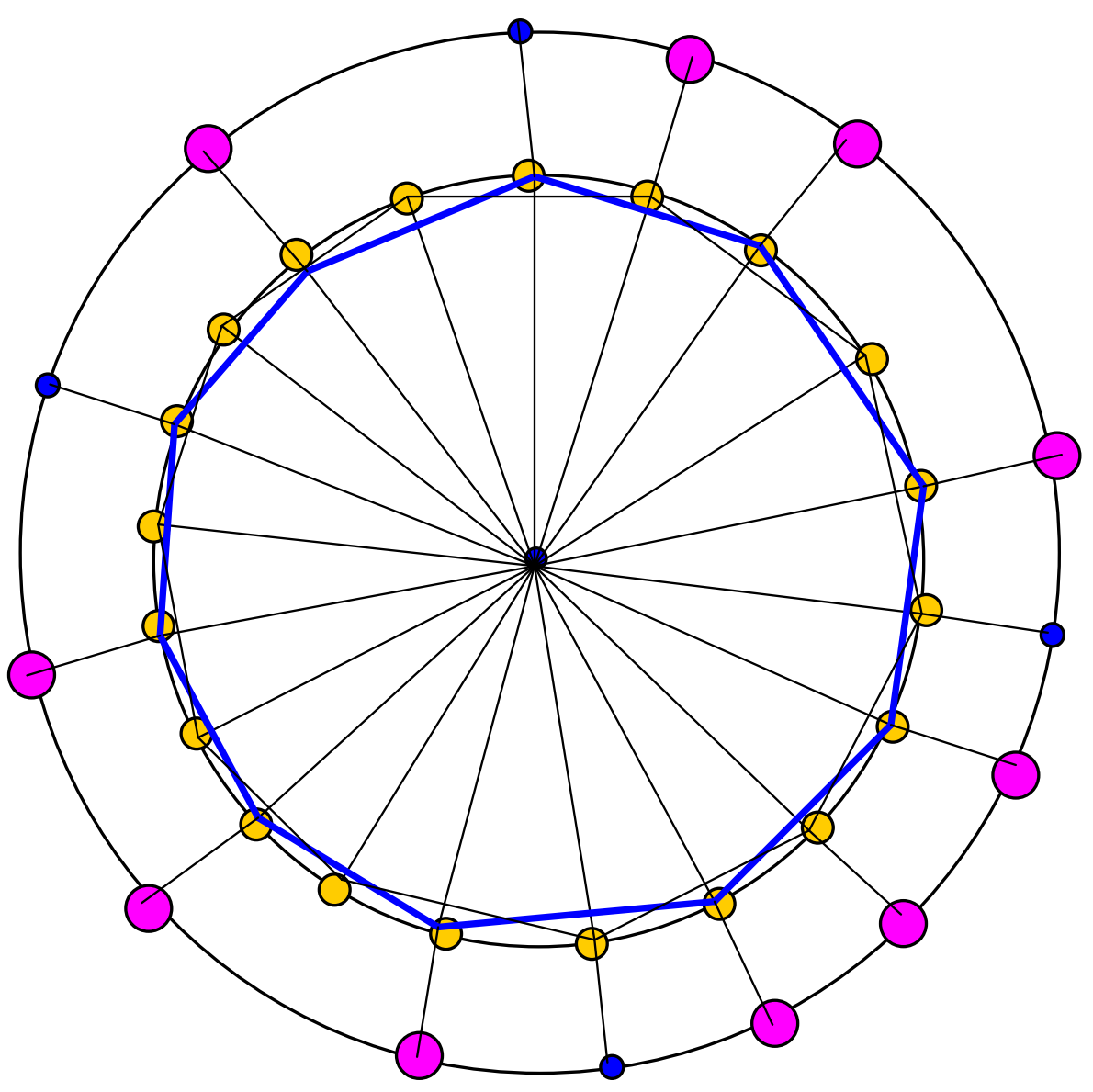




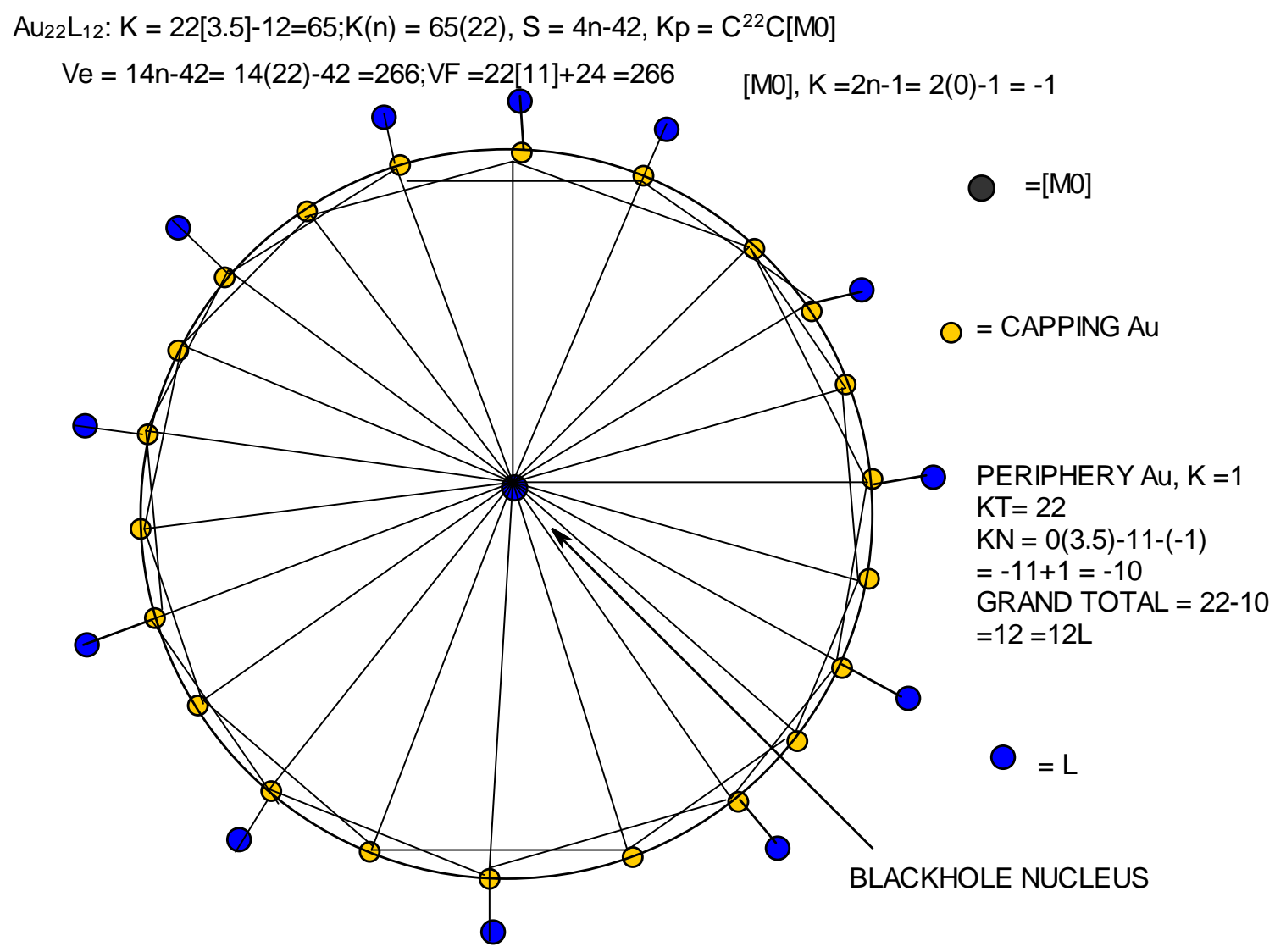

$K(n)=65(22)$ belongs to [M0] series 
$\mathrm{Au}_{16} \mathrm{~L}_{8} \mathrm{Cl}_{6}: \mathrm{K}=16[3.5]-8-3=45 ; \mathrm{K}(\mathrm{n})=45(16), \mathrm{S}=4 \mathrm{n}-26, \mathrm{Kp}=\mathrm{C}^{14} \mathrm{C}[\mathrm{M} 2]$

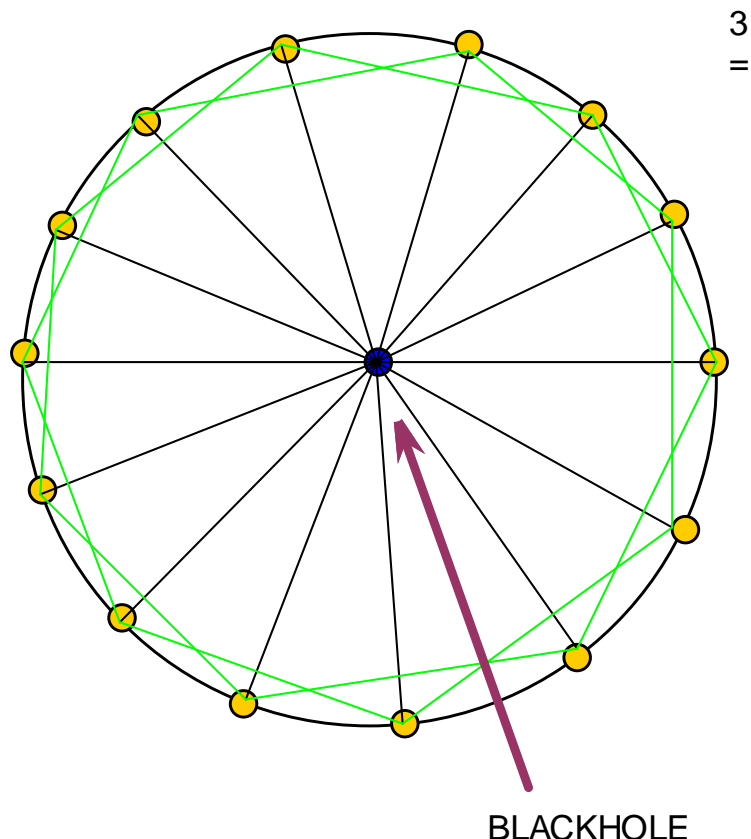

SKELETAL LINKAGES $=14+14+14+3=45$

3 ARE FROM [M2], $S=4 n+2, K=2 n-1$

$=2(2)-1=3$

Ligand linkage distribution

Periphery $=14[3.5]-14(2.5)=14$

Nucleus $=2[3.5]-14(0.5)-3=-3$

This means that the nucleus has negative nulear linkages for ligands-BLACKHOLE.

The NET linkages available for ligands =

$14+(-3)=8$. This is the same as $8 \mathrm{l}+6 \mathrm{Cl}$.

$=$ [M2] BLACKHOLE NUCLEUS

O CAPPING Au

$K(n)=45(16)$ belongs to $[M 2]$ series.

The categorization of $\mathrm{K}(\mathrm{n})$ values of the golden clusters into respective [Mx] clan groups can easily be verified using the appropriate series given in SRS-1. The calculation of K values and respective valence electrons from the series formula, Ve and the cluster formula, VF are given in Table 1. The clusters were regrouped according to [Mx] series and are presented in Table 2. In order to emphasize the idea of grouping the clusters according to [Mx] series, cluster group trees of selected clusters were constructed F-35 to F-37 for selected clusters of [M1], [M2] and [M6]. A proposed scheme for broad grouping of clusters is shown GR-1.

Table 1. Deducing K(n) Parameter and Deriving the Series of Golden Clusters

\begin{tabular}{|c|c|c|c|c|c|c|c|}
\hline CLUSTER & $\mathrm{n}$ & K VALUE & $\mathrm{K}(\mathrm{n})$ & $\begin{array}{l}\text { SERIES } \\
S=4 n+q\end{array}$ & $\begin{array}{l}\mathrm{Kp} \\
=\mathrm{C}^{\mathrm{y}} \mathrm{C}[\mathrm{Mx}]\end{array}$ & $\begin{array}{l}\mathrm{Ve} \\
\mathrm{Ve}=14 n+q\end{array}$ & VF \\
\hline $\mathrm{Au}_{4} \mathrm{I}_{2} \mathrm{~L}_{4}$ & 4 & $4[3.5]-1-4=9$ & $9(4)$ & $4 n-2$ & $\mathrm{C}^{2} \mathrm{C}[\mathrm{M} 2]$ & $14(4)-2=54$ & $4[11]+2+8=54$ \\
\hline $\mathrm{Au}_{6} \mathrm{~L}_{8}{ }^{2+}$ & 6 & $6[3.5]-8+1=14$ & $14(6)$ & $4 n-4$ & $\mathrm{C}^{3} \mathrm{C}[\mathrm{M} 3]$ & $14(6)-4=80$ & $6[11]+8(2)-2=80$ \\
\hline $\mathrm{Au}_{6} \mathrm{~L}_{6}{ }^{2+}$ & 6 & $6[3.5]-6+1=16$ & $16(6)$ & $4 n-8$ & $\mathrm{C}^{5} \mathrm{C}[\mathrm{M} 1]$ & $14(6)-8=76$ & $6[11]+6(2)-2=76$ \\
\hline $\mathrm{Au}_{7} \mathrm{~L}_{7}^{+1}$ & 7 & $7[3.5]-7+0.5=18$ & $18(7)$ & $4 n-8$ & $\mathrm{C}^{5} \mathrm{C}[\mathrm{M} 2]$ & $14(7)-8=90$ & $7[11]+14-1=90$ \\
\hline $\mathrm{Au}_{8} \mathrm{~L}_{8} \mathrm{Cl}_{2}{ }^{2+}$ & 8 & $8[3.5]-8-1+1=20$ & $20(8)$ & $4 n-8$ & $\mathrm{C}^{5} \mathrm{C}[\mathrm{M} 3]$ & $14(8)-8=104$ & $8[11]+8(2)+2-2=104$ \\
\hline $\mathrm{Au}_{8} \mathrm{~L}_{8}^{2+}$ & 8 & $8[3.5]-8+1=21$ & $21(8)$ & $4 n-10$ & $\mathrm{C}^{6} \mathrm{C}[\mathrm{M} 2]$ & $14(8)-10=102$ & $8[11]+8(2)-2=102$ \\
\hline $\mathrm{Au}_{8} \mathrm{~L}_{7}{ }^{2+}$ & 8 & $8[3.5]-7+1=22$ & $22(8)$ & $4 n-12$ & $\mathrm{C}^{7} \mathrm{C}[\mathrm{M} 1]$ & $14(8)-12=100$ & $8[11]+7(2)-2=100$ \\
\hline $\mathrm{Au}_{9} \mathrm{~L}_{8}^{+}$ & 9 & $9[3.5]-8+0.5=24$ & $24(9)$ & $4 n-12$ & $\mathrm{C}^{7} \mathrm{C}[\mathrm{M} 2]$ & $14(9)-12=114$ & $9[11]+16-1=114$ \\
\hline $\mathrm{Au}_{9} \mathrm{~L}_{8}{ }^{3+}$ & 9 & $9[3.5]-8+1.5=25$ & $25(9)$ & $4 n-14$ & $\mathrm{C}^{8} \mathrm{C}[\mathrm{M} 1]$ & $14(9)-14=112$ & $9[11]+8(2)-3=112$ \\
\hline \multicolumn{8}{|l|}{$\mathrm{L}=\mathrm{PAr} 3$} \\
\hline $\mathrm{Au}_{10} \mathrm{~L}_{8} \mathrm{Cl}^{+}$ & 10 & $10[3.5]-8-0.5+0.5=27$ & $27(10)$ & $4 n-14$ & $\mathrm{C}^{8} \mathrm{C}[\mathrm{M} 2]$ & $14(10)-14=126$ & $10[11]+16+1-1=126$ \\
\hline $\mathrm{Au}_{10} \mathrm{~L}_{6} \mathrm{Cl}_{3}^{+}$ & 10 & $10[3.5]-6-1.5+0.5=28$ & $28(10)$ & $4 n-16$ & $\mathrm{C}^{9} \mathrm{C}[\mathrm{M} 1]$ & $14(10)-16=124$ & $10[11]+6(2)+3-1=124$ \\
\hline $\mathrm{Au}_{10} \mathrm{~L}_{3} \mathrm{R}_{4}$ & 10 & $10[3.5]-3-2=30$ & $30(10)$ & $4 n-20$ & $\mathrm{C}^{11} \mathrm{C}[\mathrm{M}-1]$ & $14(10)-20=120$ & $10[11]+3(2)+4=120$ \\
\hline $\mathrm{Au}_{11} \mathrm{~L}_{12}^{3+}$ & 11 & $11[3.5]-12+1.5=28$ & $28(11)$ & $4 n-12$ & $\mathrm{C}^{7} \mathrm{C}[\mathrm{M} 4]$ & $14(11)-12=142$ & $11[11]+12(2)-3=142$ \\
\hline $\mathrm{Au}_{11} \mathrm{~L}_{8} \mathrm{Cl}_{3}$ & & $11[3.5]-8-1.5=29$ & $29(11)$ & $4 n-14$ & $\mathrm{C}^{8} \mathrm{C}[\mathrm{M} 3]$ & $14(11)-14=140$ & $11[11]+8(2)+3=140$ \\
\hline $\mathrm{Au}_{11} \mathrm{~L}_{7} \mathrm{X}_{3}$ & 11 & $11[3.5]-7-1.5=30$ & $30(11)$ & $4 n-16$ & $\mathrm{C}^{9} \mathrm{C}[\mathrm{M} 2]$ & $14(11)-16=138$ & $11[11]+7(2)+3=138$ \\
\hline $\mathrm{L}=\mathrm{PAr}_{3}$ & & $\mathrm{X}=\mathrm{Cl}$ & & & & & \\
\hline $\mathrm{Au}_{11} \mathrm{~L}_{8} \mathrm{Cl}_{2}^{+}$ & 11 & $11[3.5]-8-1+0.5=30$ & $30(11)$ & $4 n-16$ & $\mathrm{C}^{9} \mathrm{C}[\mathrm{M} 2]$ & $14(11)-16=138$ & $11[11]+8(2)+2-1=138$ \\
\hline
\end{tabular}




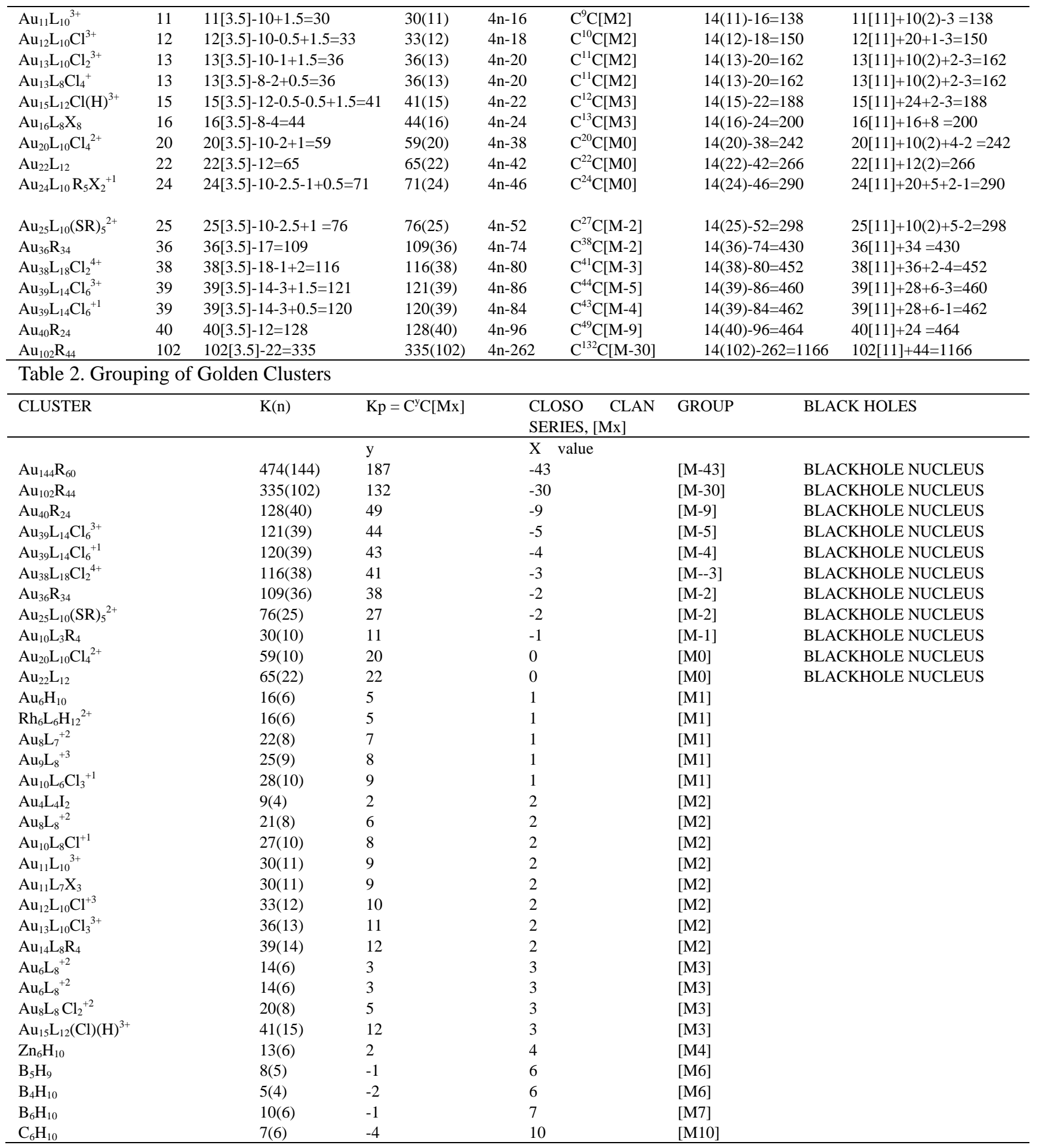




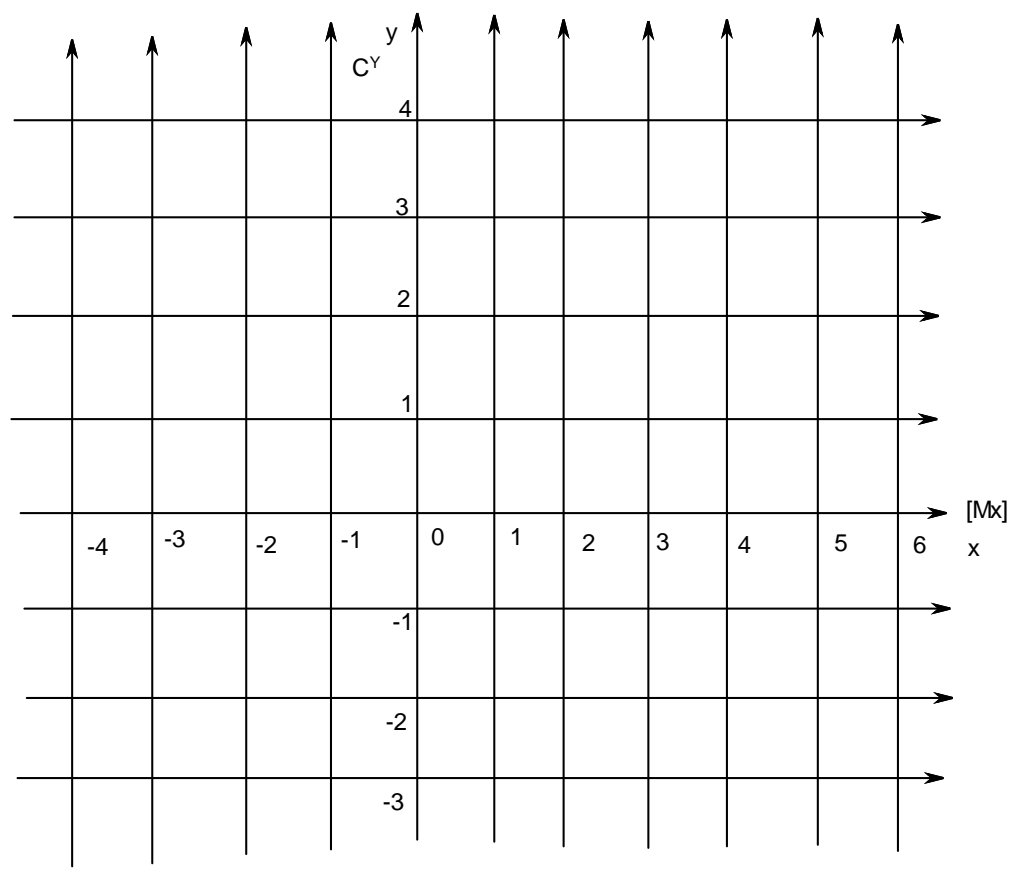

GR-1

Clan (Group) Cluster Trees

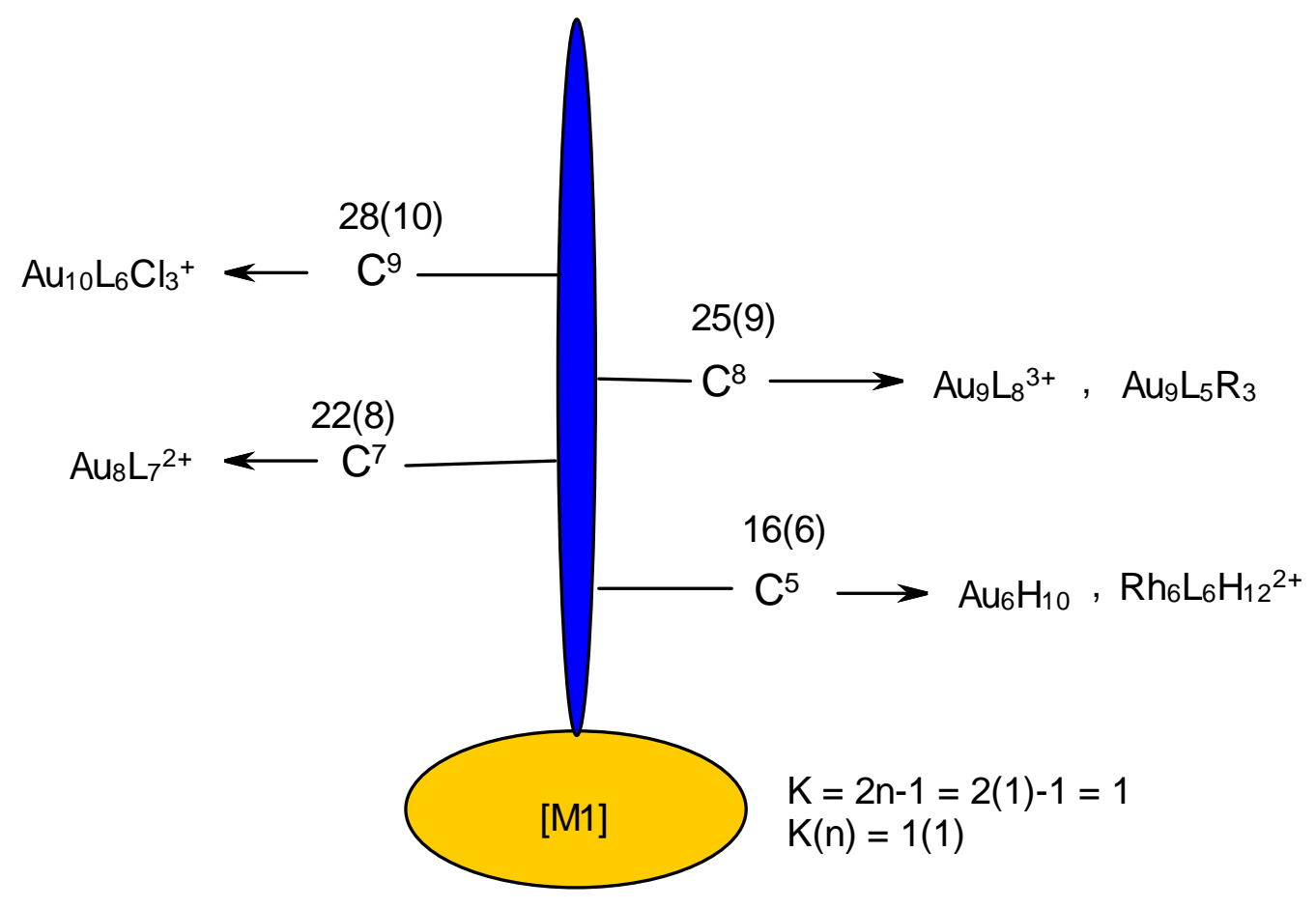

CLUSTER GROUP(CLAN) TREE 


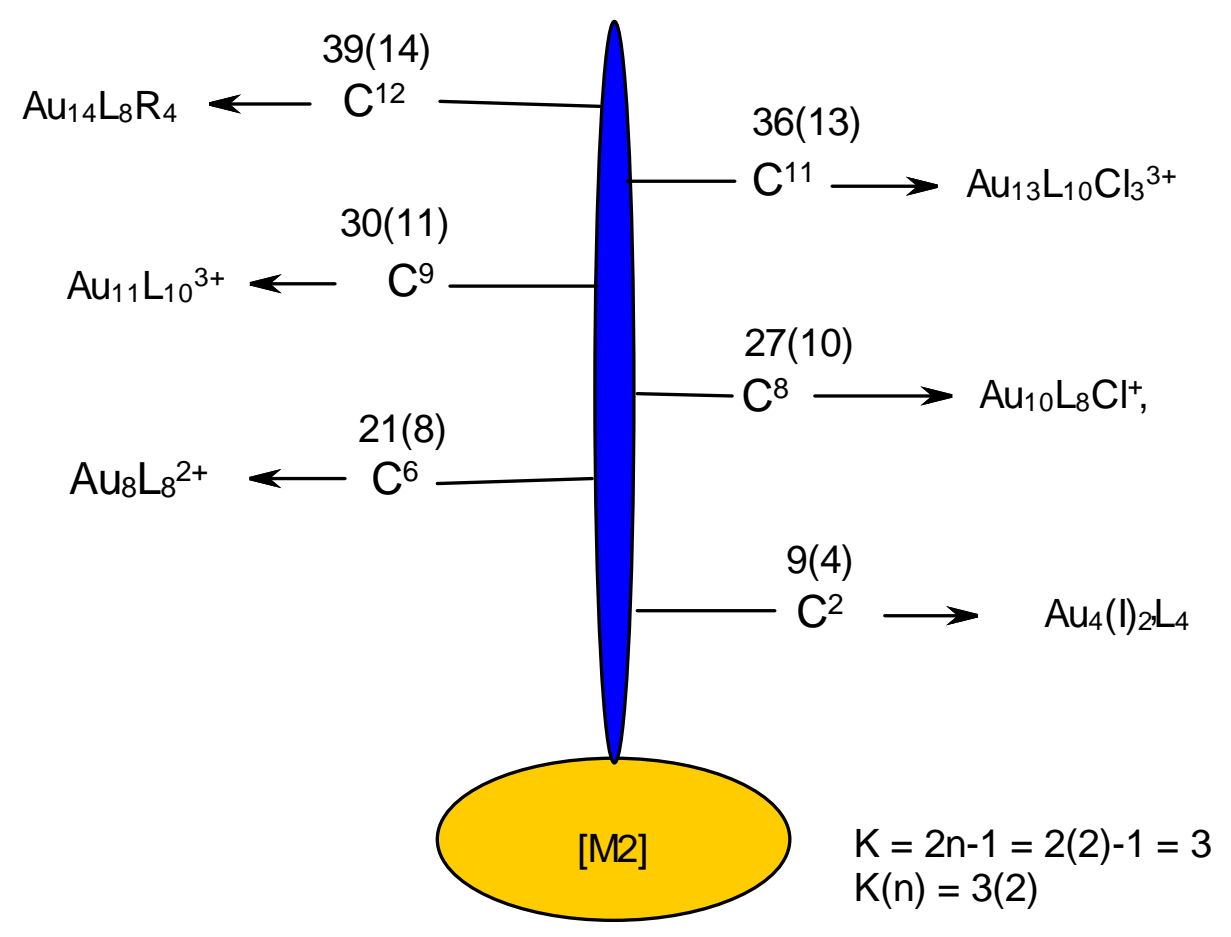

CLUSTER GROUP(CLAN) TREE

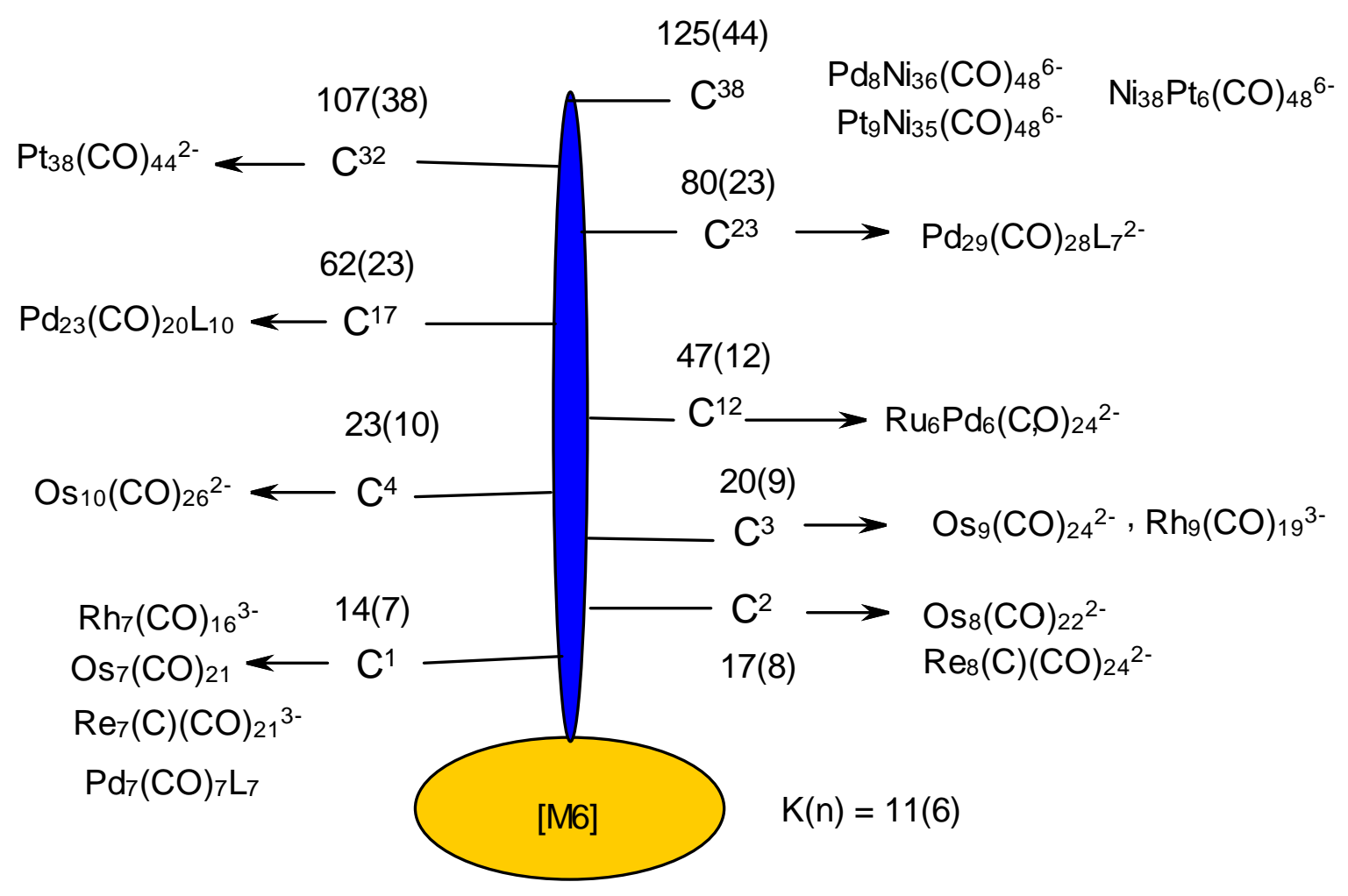

CLUSTER GROUP(CLAN) TREE 


\section{The Existance of Blackholes in the Nuclei of Some Golden Clusters}

In the previous work, it was discovered that the series method could be applied to the analysis and categorization of golden clusters (Kiremire, 2016a 2017a). It was also found that the majority of the simple clusters of nuclearity index 4-13 had either ONE or TWO golden skeletal elements in the cluster nucleus (Kiremire, 2016a, 2017a). This information was in agreement with earlier observation by the series method that indeed some clusters had octahedral cluster nuclei in them (Kiremire, 2016b).The subsequent work also revealed that, clusters could be explained more clearly in terms of their structures and ligand distribution onto skeletal elements by using skeletal numbers and valences (Kiremire, 2017c). Using skeletal numbers and the ligand distribution concept, it has been discovered that some golden cluster nuclei with [Mx], where $\mathrm{x} \leq 0$ do "swallow" skeletal and/or ligand linkages. As a consequence, the numbers of linkages left for binding to ligands are considerably diminished giving rise to some golden clusters being left naked, that is, having no ligands at all. It is proposed that clusters with nuclei which appear to "swallow" cluster linkages be regarded as NUCLEI WITH BLACKHOLES. Examples of golden clusters having nuclei with black-holes are given F-38 to F-41.

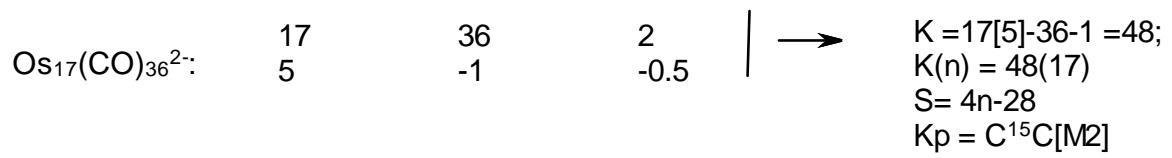

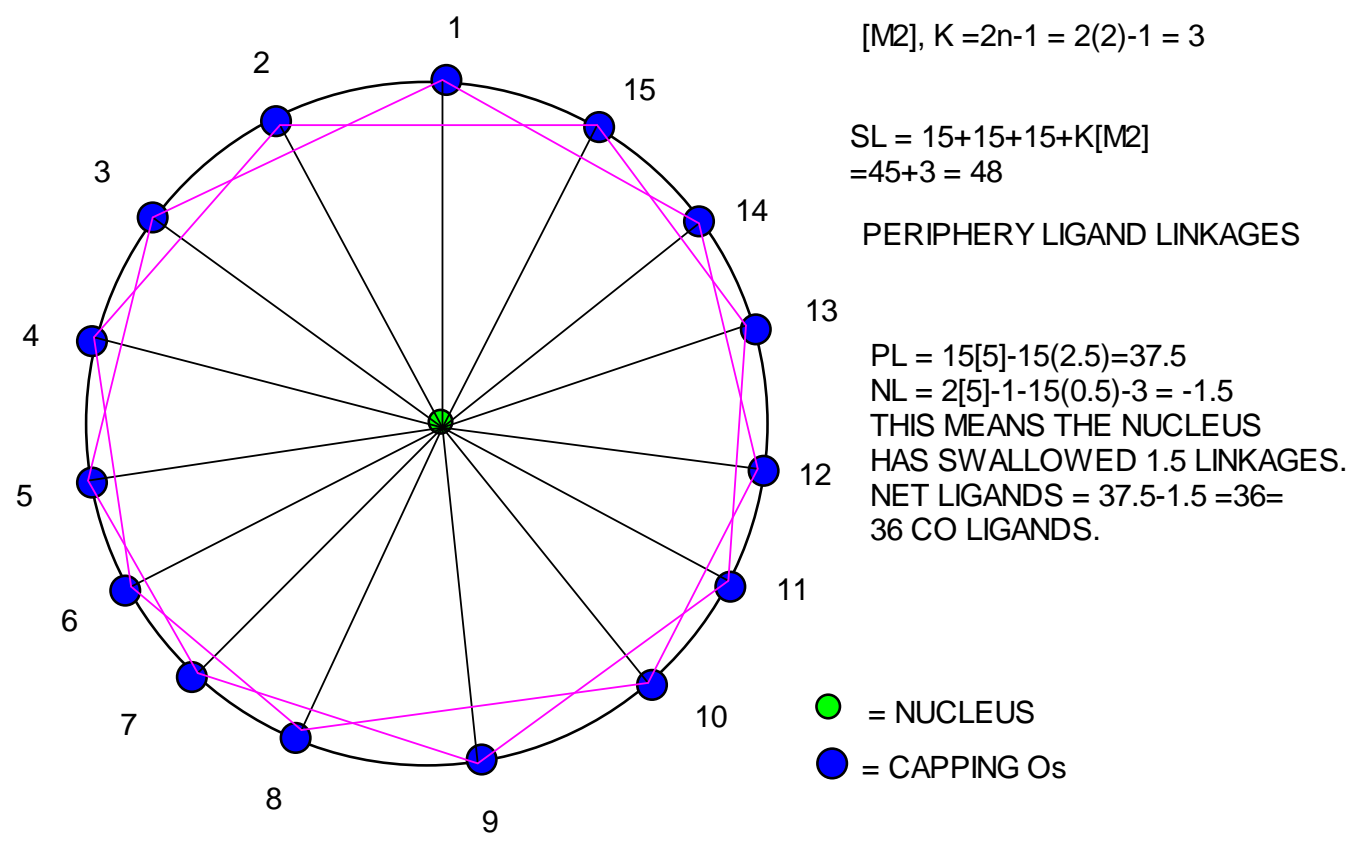




$$
\mathrm{Os}_{20}(\mathrm{CO}) 40^{2-:} \quad \begin{aligned}
& 20402 \\
& 5-1-0.5
\end{aligned}
$$

BLACK HOLE

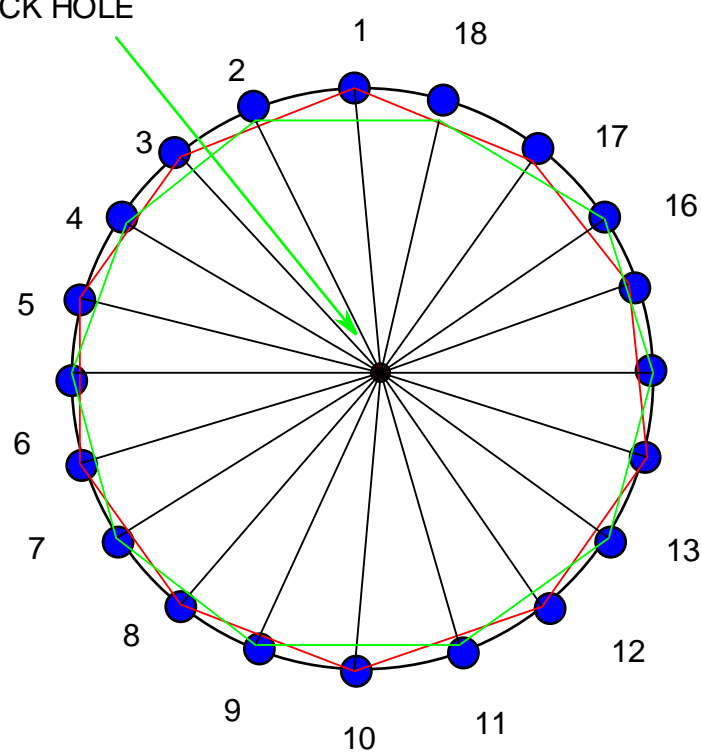

- = NUCLEUS WITH A BLACK HOLE

$=$ CAPPING OS SKELETAL ELEMENTS
$\mathrm{K}=20[5]-40-1=59$

$\mathrm{K}(\mathrm{n})=59(20)$

$\mathrm{S}=4 \mathrm{n}-38$

$\mathrm{Kp}=\mathrm{C}^{20} \mathrm{C}[\mathrm{MO}]$

$\mathrm{SL}=20(3)=60$

$\mathrm{KN}=2 \mathrm{n}-1=2(0)-1=-1$

$\mathrm{K}=60-1=59$

\section{LIGAND LINKAGES}

PERIPHERY

$=20(5)-20(2.5)=50$

NUCLEUS

$\mathrm{KN}=0(5)-1-20(0.5)-[-1]$

$=-10$

$\mathrm{NET}=50-10=40$

$=40 \mathrm{CO}$ LIGANDS

$\mathrm{KN}=-10$, COULD BE REGARDED

AS THE SWALLOWING OF CLUSTER

LINKAGES. WE MAY REGARD THE

NUCLEUS TO BE BEHAVING AS A BLACK

HOLE. 


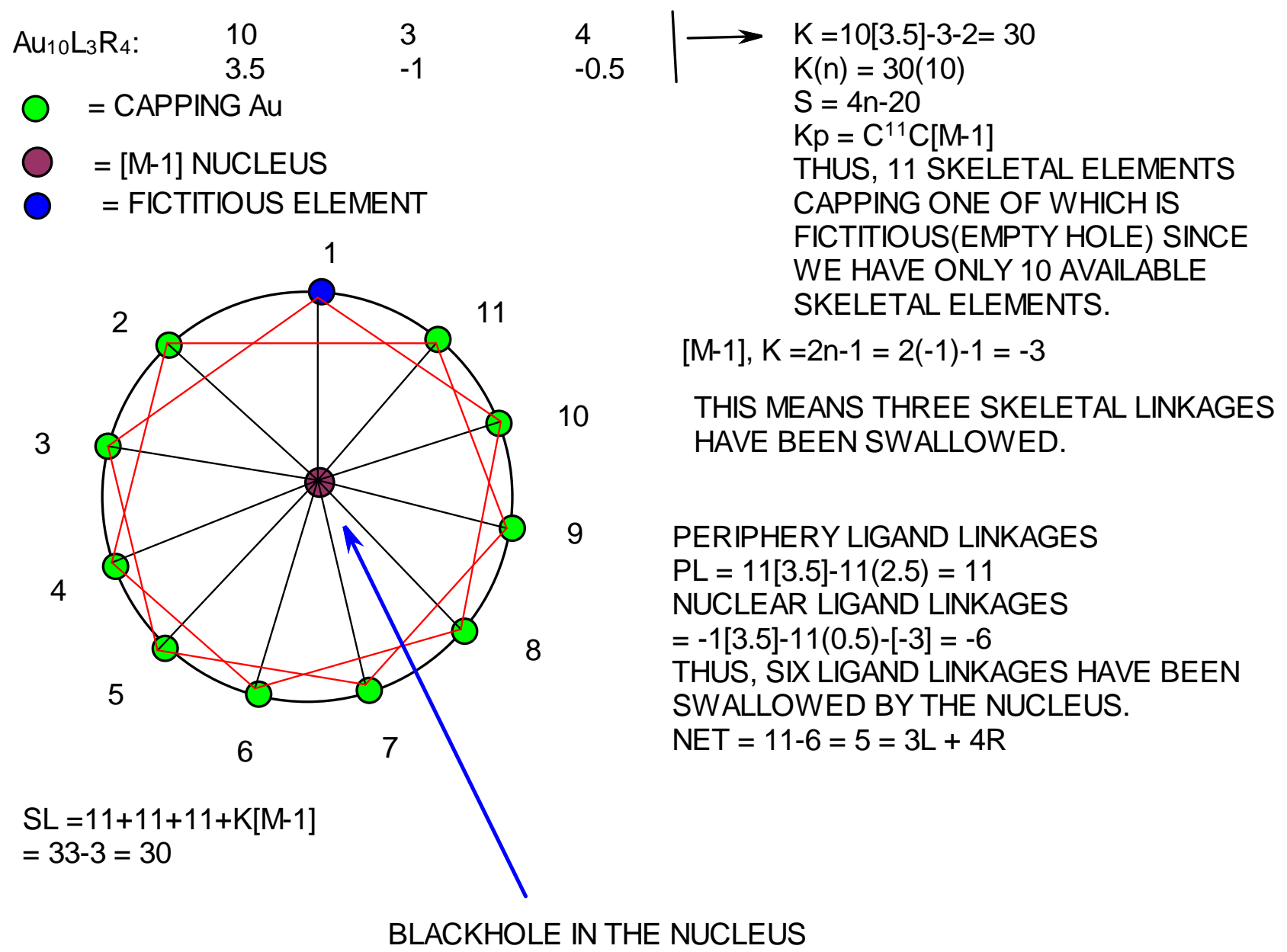




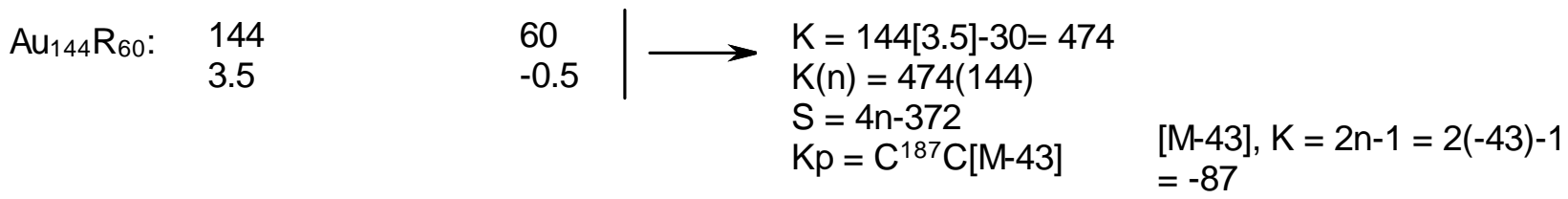

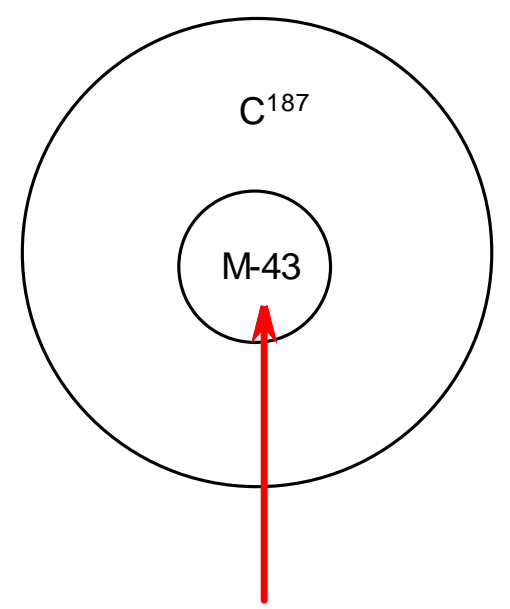

SKELETAL LINKAGES, SL= $187(3)+[2(-43)-1]=474$

PERIPHERY LIGAND LINKAGES, $\mathrm{PL}=187[3.5]-187(2.5)=187$

NUCLEAR LIGAND LINKAGES $=-43[3.5]-187(0.5)-[-87]=-157$ $\mathrm{NET}=187-157=30=60 \mathrm{R}$

BLACKHOLE NUCLEUS

Table 3. Data Interpretation

\begin{tabular}{|c|c|c|c|c|c|c|c|c|}
\hline & & $\mathrm{K}$ & $\mathrm{K}(\mathrm{n})$ & SERIES & Kp & $\mathrm{Ve}$ & $\mathrm{VF}$ & RESULT \\
\hline 1 & $\mathrm{Au}_{6} \mathrm{~L}_{8}{ }^{2+}$ & $6[3.5]-8+1=14$ & $14(6)$ & $4 n-4$ & $\mathrm{C}^{\frac{3}{3}} \mathrm{C}[\mathrm{M} 3]$ & $14(6)-4=80$ & $6[11]+16-2=80$ & Different \\
\hline 2 & $\mathrm{Au}_{7} \mathrm{~L}_{8}{ }^{3+}$ & $7[3.5]-8+1.5=18$ & 18(7) & $4 n-8$ & $\mathrm{C}^{5} \mathrm{C}[\mathrm{M} 2]$ & $14(7)-8=90$ & $7[11]+16-3=90$ & Different \\
\hline 3 & $\mathrm{Au}_{6} \mathrm{~L}_{8}{ }^{2+}$ & $6[3.5]-8+1=14$ & $14(6)$ & $4 n-4$ & $\mathrm{C}^{3} \mathrm{C}[\mathrm{M} 3]$ & $14(6)-4=80$ & $6[11]+16-2=80$ & Different \\
\hline 4 & $\mathrm{Au}_{8} \mathrm{~L}_{8} \mathrm{Cl}_{2}^{2+}$ & $8[3.5]-8-1+1=20$ & $20(8)$ & $4 n-8$ & $\mathrm{C}^{5} \mathrm{C}[\mathrm{M} 3]$ & $14(8)-8=104$ & $8[11]+16+2-2=104$ & Different \\
\hline 5 & $\mathrm{Au}_{11} \mathrm{~L}_{12}^{3+}$ & $11[3.5]-12+1.5=28$ & $28(11)$ & $4 n-12$ & $\mathrm{C}^{7} \mathrm{C}[\mathrm{M} 4]$ & $14(11)-12=142$ & $11[11]+24-3=142$ & Different \\
\hline 6 & $\mathrm{Au}_{11} \mathrm{~L}_{7} \mathrm{Cl}_{3}$ & $11[3.5]-7-1.5=30$ & $30(11)$ & $4 n-16$ & $\mathrm{C}^{9} \mathrm{C}[\mathrm{M} 2]$ & $14(11)-16=138$ & $11[11]+14+3=138$ & Similar \\
\hline 7 & $\mathrm{Au}_{11} \mathrm{~L}_{8} \mathrm{Cl}_{2}^{+}$ & $11[3.5]-8-1+0.5=30$ & $30(11)$ & $4 n-16$ & $\mathrm{C}^{9} \mathrm{C}[\mathrm{M} 2]$ & 138 & $11[11]+16+2-1=138$ & Similar \\
\hline 8 & $\mathrm{Au}_{20} \mathrm{~L}_{8}^{4+}$ & $20[3.5]-8+2=64$ & $64(20)$ & $4 n-48$ & $\mathrm{C}^{25} \mathrm{C}[\mathrm{M}-5]$ & $14(20)-48=232$ & $20[11]+16-4=232$ & Similar? \\
\hline 9 & $\mathrm{Au}_{13} \mathrm{~L}_{10} \mathrm{Cl}_{2}^{3+}$ & $13[3.5]-10-1+1.5=36$ & $36(13)$ & $4 n-20$ & $\mathrm{C}^{11} \mathrm{C}[\mathrm{M} 2]$ & $14(13)-20=162$ & $13[11]+20+2-3=162$ & Similar? \\
\hline 10 & $\mathrm{Au}_{22} \mathrm{~L}_{12}$ & $22[3.5]-12=65$ & $65(22)$ & $4 n-42$ & $\mathrm{C}^{22} \mathrm{C}[\mathrm{M} 0]$ & $14(22)-42=266$ & $22[11]+24=266$ & Different \\
\hline 11 & $\mathrm{Au}_{22} \mathrm{~L}_{14}$ & $22[3.5]-14=63$ & $63(22)$ & $4 n-38$ & $\mathrm{C}^{20} \mathrm{C}[\mathrm{M} 2]$ & $14(22)-38=270$ & $22[11]+28=270$ & Different \\
\hline
\end{tabular}

\section{Data Interpretation}

A number of golden clusters have been studied by UV-Vis-NID method (Wang, et al, 2016). For the purposes of illustrating how the series method analysis of clusters could be utilized, these findings are given in Table 3 . For ease of differentiation of the clusters and grouping them, let us refer to the clusters with the same closo nucleus $[\mathrm{Mx}]$ as being in the same CLUSTER GROUP but different series $S=4 n+q(q$ varies), while those which belong to the same series $S=$ $4 \mathrm{n}+\mathrm{q}$ ( $\mathrm{q}$ is the same) as being in the same CLUSTER PERIOD. This approach is similar to what we have in the ordinary periodic table of elements. Let us look at the following clusters: $\mathrm{Pd}_{10}(\mathrm{CO})_{12} \mathrm{~L}_{6} ; \mathrm{K}(\mathrm{n})=22(10), \mathrm{S}=4 \mathrm{n}-4, \mathrm{Kp}=$ $\left.\mathrm{C}^{3} \mathrm{C}[\mathrm{M} 7] ; \mathrm{Pd}_{23}(\mathrm{CO})_{21} \mathrm{~L}_{10} ; \mathrm{K}=61(23), \mathrm{S}=4 \mathrm{n}-30, \mathrm{Kp}=\mathrm{C}^{16} \mathrm{CM} 7\right]$ and $\mathrm{Au}_{4} \mathrm{Pd}_{28}(\mathrm{CO})_{22} \mathrm{~L}_{16} ; \mathrm{K}=88(32), \mathrm{S}=4 \mathrm{n}-48, \mathrm{Kp}=$ $\mathrm{C}^{25} \mathrm{C}[\mathrm{M} 7]$. All these three clusters possess the similar cluster nucleus [M7] but different number of capping elements. These clusters are regarded as being in the same CLUSTER GROUP but different cluster capping periods-one has 3 capping skeletal elements, the other 16 capping elements and lastly the other has 25 capping elements. Let us take another example: $\mathrm{Pd}_{29}(\mathrm{CO})_{28} \mathrm{~L}_{7}{ }^{2-}: \mathrm{Pd}_{30}(\mathrm{CO})_{26} \mathrm{~L}_{10}: \mathrm{K}=84, \mathrm{~K}(\mathrm{n})=84(30), \mathrm{S}=4 \mathrm{n}-48, \mathrm{Kp}=\mathrm{C}^{25} \mathrm{C}[\mathrm{M} 5]$ and $\mathrm{Au}_{4} \mathrm{Pd}_{28}(\mathrm{CO})_{22} \mathrm{~L}_{16} ; \mathrm{K}=88(32), \mathrm{S}=4 \mathrm{n}-48, \mathrm{Kp}=\mathrm{C}^{25} \mathrm{C}[\mathrm{M} 7]$. These two clusters belong to the same CLUSTER PERIOD, $\mathrm{S}$ $=4 \mathrm{n}-48$. They have the same number of capping elements but different nuclear sizes, one with 5 skeletal elements in the nucleus and the other 7. With this proposed definition of CLUSTER GROUP and CLUSTER PERIOD introduced, we 
can now have some idea or understanding of the results indicated in the table. According to the series method, clusters 1 $\left\{[\mathrm{M} 3], \mathrm{C}^{3}\right\}$ and $2\left\{[\mathrm{M} 2], \mathrm{C}^{5}\right\}$ :-these two belong to different cluster groups and cluster periods;clusters $3\left\{[\mathrm{M} 3], \mathrm{C}^{3}\right\}$, $4\left\{[\mathrm{M} 3], \mathrm{C}^{5}\right\}$, and5\{[M4], $\left.\mathrm{C}^{7}\right\}$ :- cluster 3 and 4 belong to the same group but different periods while cluster 5 has nothing in common with clusters 3 and $4 ; 6\left\{[\mathrm{M} 2], \mathrm{C}^{9}\right\}$ and $7\left\{[\mathrm{M} 2], \mathrm{C}^{9}\right\}$ :-clusters 6 and 7 belong to the same group and period; $8\left\{[\mathrm{M}-5], \mathrm{C}^{25}\right\}, 9\left\{[\mathrm{M} 2], \mathrm{C}^{11}\right\}$ :-clusters 8 and 9 have nothing in common; $10\left\{[\mathrm{M} 0], \mathrm{C}^{22}\right\}$ and $11\left\{[\mathrm{M} 2], \mathrm{C}^{20}\right\}$ :clusters 10 and 11 are also different as they have nothing in common.

\section{CONVERSION OF $\mathrm{A}_{9} \mathrm{~L}_{8}{ }^{3+}$ CLUSTER TO $\mathrm{Au}_{9} \mathrm{~L}_{8}{ }^{+}$CLUSTER}

The change of skeletal structure of $\mathrm{A}_{9} \mathrm{~L}_{8}{ }^{3+}$ to a different one of $\mathrm{Au}_{9} \mathrm{~L}_{8}{ }^{+}$was reported by Mingos (Mingos, 1984). This transformation can also be explained by the series approach. The $\mathrm{K}(\mathrm{n})$ value of $\mathrm{Au}_{9} \mathrm{~L}_{8}{ }^{3+}$ is 25(9) while that of $\mathrm{Au}_{9} \mathrm{~L}_{8}{ }^{+}$is 24(9). The decrease in $\mathrm{K}$ value by 1 results in a decrease of skeletal capping by 1 with a parallel increase in the nuclear index by 1 . That is, instead of the geometry having 8 capping elements and 1 in the nucleus it changes into another on having 7 capping skeletal elements and 2 nuclear elements. In terms of cluster grouping proposed, the cluster has moved from CLUSTER GROUP 1[M1] to CLUSTER GROUP 2[M2]. This is illustrated in F-42.

A

\begin{tabular}{l}
\multicolumn{1}{c}{\begin{tabular}{c}
\multicolumn{1}{c}{$A$} \\
AugL8
\end{tabular}${ }^{3+}$} \\
$\mathrm{K}=9[3.5]-8+1.5=25$ \\
$\mathrm{~K}(\mathrm{n})=25(9)$ \\
$\mathrm{S}=4 \mathrm{n}-14$ \\
$\mathrm{Kp}=\mathrm{C}^{8} \mathrm{C}[\mathrm{M} 1]$ \\
One skeletal element \\
in the 'nucleus' \\
surrounded by 8 \\
capping skeletal \\
elements.
\end{tabular}

A

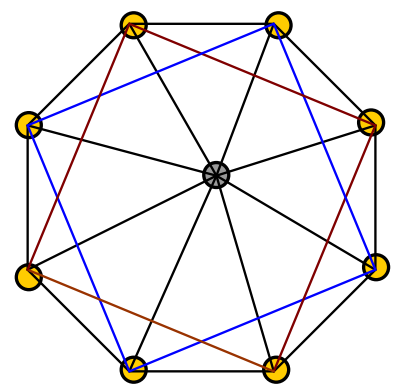

$\mathrm{SL}=8+8+8+1=25$

$$
\mathrm{O}=[\mathrm{M} 1]
$$

[M1], $S=4 n+2, K=2 n-1=2(1)-1=1$
B

$$
\begin{aligned}
& \text { AugL8 }{ }^{+} \\
& \mathrm{K}=9[3.5]-8+0.5=24 \\
& \mathrm{~K}(\mathrm{n})=24(9) \\
& \mathrm{S}=4 \mathrm{n}-12 \\
& \mathrm{Kp}=\mathrm{C}^{7} \mathrm{C}[\mathrm{M} 2] \\
& \text { Two skeletal elements in the 'nucleus' } \\
& \text { surrounded by } 7 \text { skeletal elements. }
\end{aligned}
$$

$$
S L=\text { SKELETAL LINKAGES }
$$

B

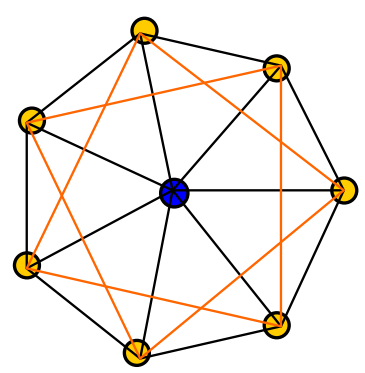

$$
\mathrm{SL}=7+7+7+3=24
$$

$=[\mathrm{M} 2]$

[M2], $S=4 n+2, K=2 n-1=2(2)-1=3$

\section{Cluster Isomerism}

Skeletal elements with the same nuclearity index (n) and same skeletal number K have been found to portray different geometrical shapes. We can refer to this as K ISOMERISM. This has been observed (Hughes\& Wade,2000) for $\mathrm{Os}_{6}(\mathrm{CO})_{18}{ }^{2-}[\mathrm{K}=11]$ and $\mathrm{Os}_{6}(\mathrm{H})_{2}(\mathrm{CO})_{18}[\mathrm{~K}=11]$ in $\mathrm{F}-43$. The encounter with $\mathrm{K}$ isomerism in golden cluster $\mathrm{A}_{9} \mathrm{~L}_{8}{ }^{3+}$ (Mingos, 1984) is not a surprise. The rough sketches of the isomerism are illustrated in F-43 to F-48. 
$\mathrm{Os}_{6}(\mathrm{CO})_{18^{2-}}$

$\mathrm{K}=6[5]-18-1=11$

$\mathrm{K}(\mathrm{n})=11(6)$

$S=4 n+2$

CLOSO; [M6]

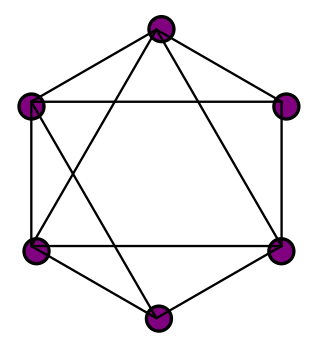

$\mathrm{K}=11$

\section{K- ISOMERISM}

$\mathrm{Os}_{6}(\mathrm{H})_{2}(\mathrm{CO})_{18}$

$\mathrm{K}=6[5]-1-18=11$

$\mathrm{K}(\mathrm{n})=11(6)$

$S=4 n+2$

CLOSO, [M6]

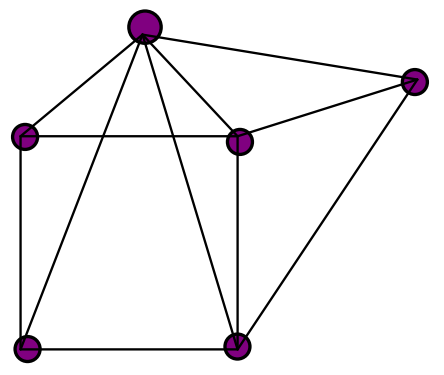

$K=8+3=11$

Aug $\left.\mathrm{L}_{8}\right]\left(\mathrm{NO}_{3}\right)_{3} \quad \mathrm{~L}=\mathrm{P}\left(\mathrm{p}-\mathrm{C}_{6} \mathrm{H}_{4} \mathrm{OMe}\right)_{3}$

ISOMERISM

$\mathrm{Kp}=\mathrm{C}^{8} \mathrm{C}[\mathrm{M} 1] \quad \mathrm{K}(\mathrm{n})=25(9)$

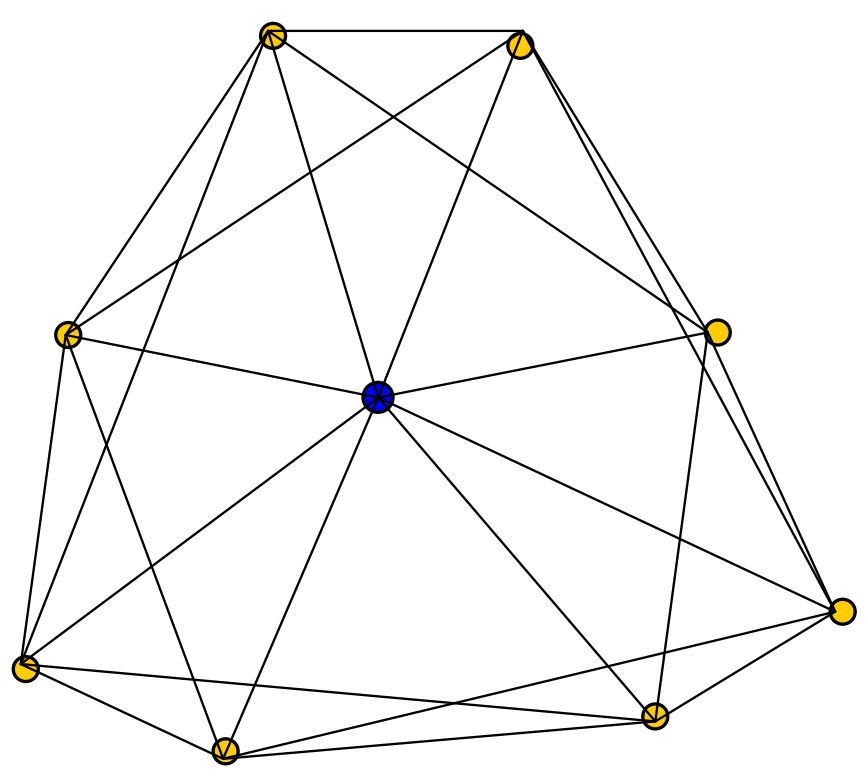


Aug $\left.L_{8}\right]\left(\mathrm{NO}_{3}\right)_{3} \quad \mathrm{~L}=\mathrm{P}\left(\mathrm{p}-\mathrm{C}_{6} \mathrm{H}_{4} \mathrm{OMe}\right)_{3}$

ISOMERISM

$\mathrm{Kp}=\mathrm{C}^{8} \mathrm{C}[\mathrm{M} 1] \quad \mathrm{K}(\mathrm{n})=25(9)$

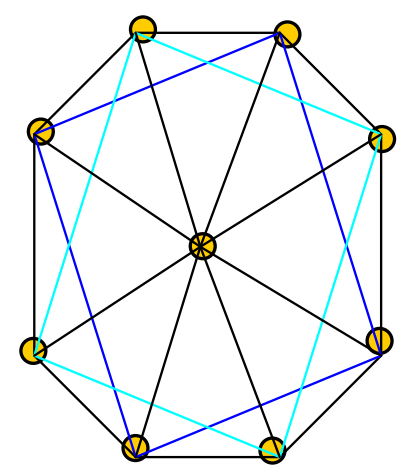

$\mathrm{Au}_{16} \mathrm{~L}_{8} \mathrm{Cl}_{6}: \mathrm{K}=16[3.5]-8-3=45 ; \mathrm{K}(\mathrm{n})=45(16), \mathrm{S}=4 \mathrm{n}-26, \mathrm{Kp}=\mathrm{C}^{14} \mathrm{C}[\mathrm{M} 2]$

$K$ ISOMERISM PRESENT AS WELL AS A BLACKHOLE NUCLEUS

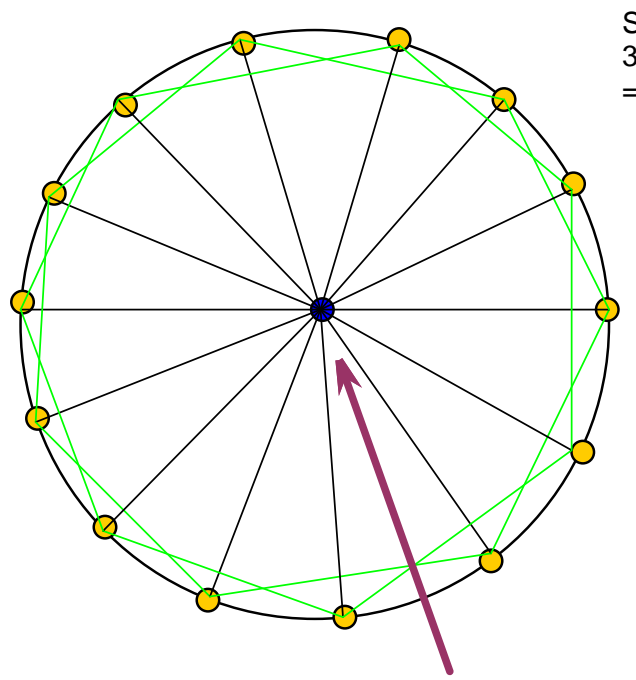

SKELETAL LINKAGES $=14+14+14+3=45$

3 ARE FROM [M2], $S=4 n+2, K=2 n-1$

$=2(2)-1=3$

Ligand linkage distribution

Periphery $=14[3.5]-14(2.5)=14$

Nucleus $=2[3.5]-14(0.5)-3=-3$

This means that the nucleus has negative nulear linkages for ligands-BLACKHOLE.

The NET linkages available for ligands $=$

$14+(-3)=8$. This is the same as $8 \mathrm{l}+6 \mathrm{Cl}$.

- $=$ [M2] BLACKHOLE NUCLEUS

$\mathrm{O}=\mathrm{CAPPING} \mathrm{Au}$

BLACKHOLE 
$\mathrm{Au}_{16} \mathrm{~L}_{8} \mathrm{Cl}_{6}: \mathrm{K}=16[3.5]-8-3=45 ; \mathrm{K}(\mathrm{n})=45(16), \mathrm{S}=4 \mathrm{n}-26, \mathrm{Kp}=\mathrm{C}^{14} \mathrm{C}[\mathrm{M} 2]$

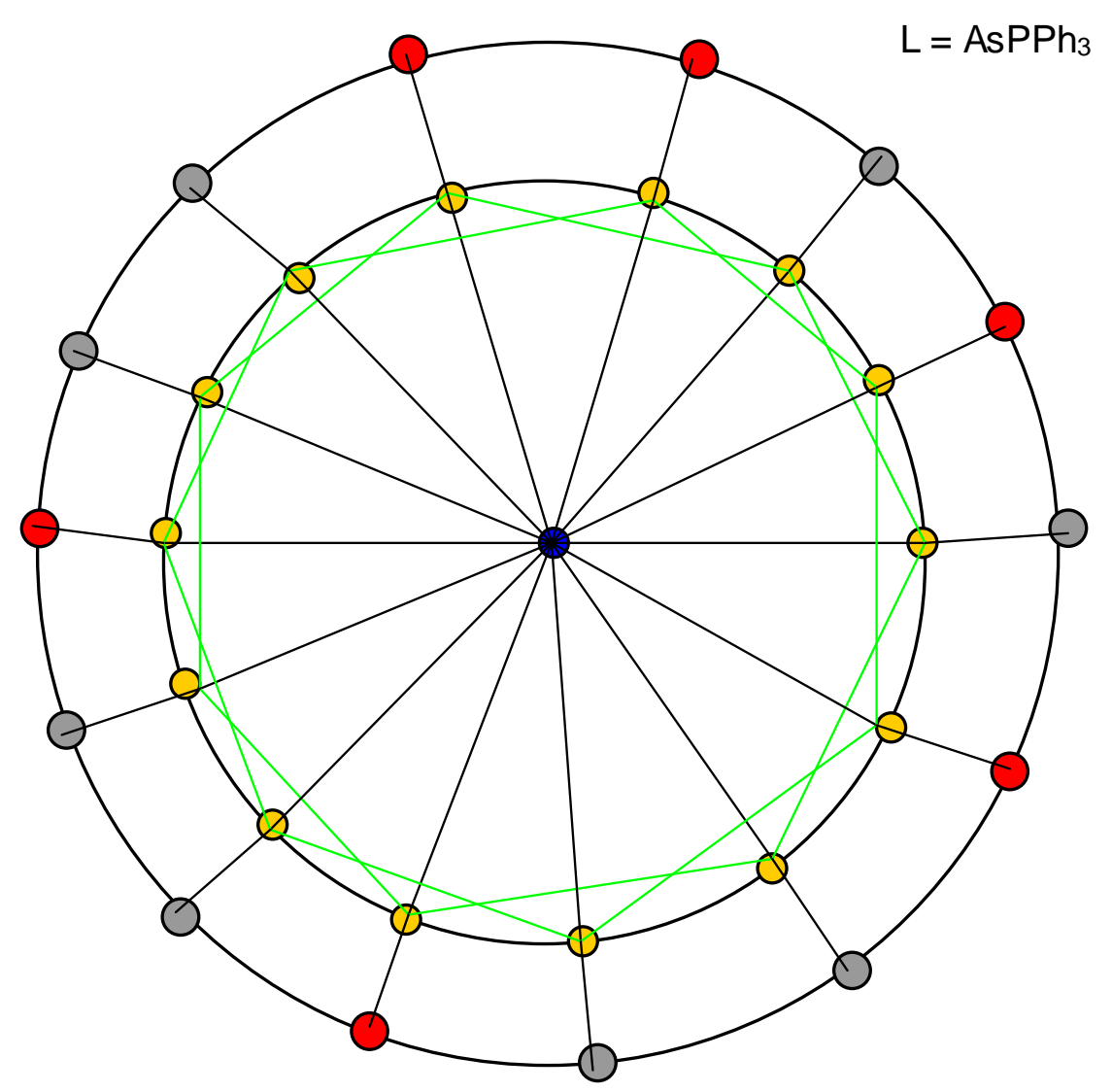


$\mathrm{Au}_{16} \mathrm{~L}_{8} \mathrm{Cl}_{6}: \mathrm{K}=16[3.5]-8-3=45 ; \mathrm{K}(\mathrm{n})=45(16), \mathrm{S}=4 n-26, \mathrm{Kp}=\mathrm{C}^{14} \mathrm{C}[\mathrm{M} 2]$

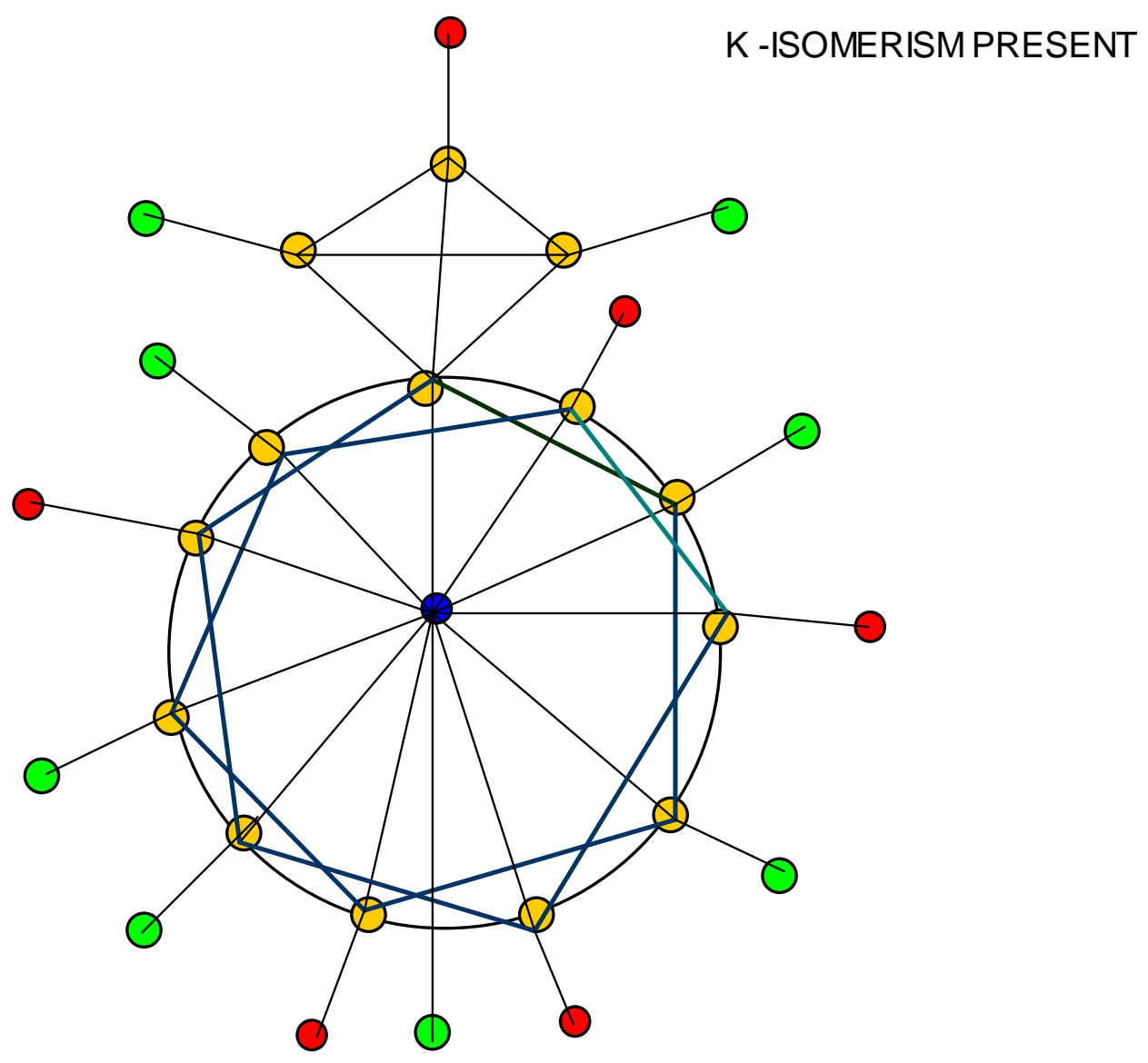

POSSIBLE LIGAND DISTRIBUTIONS

\section{DECAPPING CLUSTER SERIES:-Kp $=\mathrm{C}^{-\mathrm{y}} \mathrm{C}[\mathrm{Mx}]$ capping}

During the course of developing the $14 \mathrm{n} / 4 \mathrm{n}$ series method for categorizing clusters, a capping symbol $\mathrm{Kp}=\mathrm{C}^{\mathrm{Y}} \mathrm{C}[\mathrm{Mx}]$ was introduced. This is easily illustrated by the scheme SC-7.

SC-7

\begin{tabular}{lllllllll}
\hline $\mathrm{S}=4 \mathrm{n}+\mathrm{q}$ & $4 \mathrm{n}+10$ & $4 \mathrm{n}+8$ & $4 \mathrm{n}+6$ & $4 \mathrm{n}+4$ & $4 \mathrm{n}+2$ & $4 \mathrm{n}+0$ & $4 \mathrm{n}-2$ & $4 \mathrm{n}-4$ \\
\hline \multirow{2}{*}{$=\mathrm{C}^{\mathrm{y}} \mathrm{C}[\mathrm{Mx}]$} & KLADO & HYPHO & ARACHNO & NIDO & CLOSO & MONOCP & BICAP & TRICAP \\
& $\mathbf{C}^{-4} \mathbf{C}$ & $\mathbf{C}^{-3} \mathbf{C}$ & $\mathbf{C}^{-2} \mathbf{C}$ & $\mathbf{C}^{-1} \mathbf{C}$ & $\mathbf{C}^{0} \mathbf{C}$ & $\mathrm{C}^{1} \mathrm{C}$ & $\mathrm{C}^{2} \mathrm{C}$ & $\mathrm{C}^{3} \mathrm{C}$ \\
& $4 \mathrm{n}-6$ & $4 \mathrm{n}-8$ & $4 \mathrm{n}-10$ & $4 \mathrm{n}-12$ & $4 \mathrm{n}-14$ & $4 \mathrm{n}-16$ & $4 \mathrm{n}-18$ & $4 \mathrm{n}-20$ \\
& TETRACP & PENTACP & HEXACP & & & & & \\
$\mathrm{Kp}=\mathrm{C}^{\mathrm{y}} \mathrm{C}[\mathrm{Mx}]$ & $\mathrm{C}^{4} \mathrm{C}$ & $\mathrm{C}^{5} \mathrm{C}$ & $\mathrm{C}^{6} \mathrm{C}$ & $\mathrm{C}^{7} \mathrm{C}$ & $\mathrm{C}^{8} \mathrm{C}$ & $\mathrm{C}^{9} \mathrm{C}$ & $\mathrm{C}^{10} \mathrm{C}$ & $\mathrm{C}^{11} \mathrm{C}$ \\
\hline
\end{tabular}

Previously, the series were capturing clusters which were mono-capped, bi-capped, tri-capped, and so on (Kiremire, 2015, 2016b). The symbol was not extended to the lower series such as CLOSO, NIDO, ARACHNO, and KLAPO. According to the series, the capping or de-capping step involves one skeletal element $(\Delta \mathrm{n}=1)$ and three skeletal linkages $(\Delta K=3)$. Looking at the symbol, $K p=C^{y} C[M x], y=1$ when $S=4 n+0$, then $S=4 n-2, y=2,4 n-4, y=3$, etc. Moving backwards, from $S=4 n+0, y=1$, then for $S=4 n+2$, we can set the value of $y=0$ and so the capping symbol will be $\mathrm{Kp}=\mathrm{C}^{0} \mathrm{C}[\mathrm{Mx}]$. Further movement backwards to $\mathrm{S}=4 \mathrm{n}+4, \mathrm{y}=-1$, hence, $\mathrm{Kp}=\mathrm{C}^{-1} \mathrm{C}[\mathrm{Mx}]$. More backward movement, the capping symbol will be as indicated in the Table 4 and SC-7. The table numerically represents the concept (Rudolph, 1976) of correlating borane polyhedral geometrical structures. 
Table 4. Decapping Series

\begin{tabular}{|c|c|c|c|c|c|c|c|c|}
\hline & [M12] & $\mathbf{K}(\mathbf{n})$ & [M11] & $\mathbf{K}(\mathbf{n})$ & [M10] & $\mathbf{K}(\mathbf{n})$ & [M9] & $\mathbf{K}(\mathbf{n})$ \\
\hline $\mathrm{C}^{0}$ & $\mathrm{C}^{0} \mathrm{C}[\mathrm{M} 12]$ & $23(12)$ & $\mathrm{C}^{0} \mathrm{C}[\mathrm{M} 11]$ & $21(11)$ & $\mathrm{C}^{0} \mathrm{C}[\mathrm{M} 10]$ & 19(10) & $\mathrm{C}^{0} \mathrm{C}[\mathrm{M} 9]$ & $17(9)$ \\
\hline $\mathrm{C}^{-1}$ & $\mathrm{C}^{-1} \mathrm{C}[\mathrm{M} 12]$ & $20(11)$ & $\mathrm{C}^{-1} \mathrm{C}[\mathrm{M} 11]$ & $18(10)$ & $\mathrm{C}^{-1} \mathrm{C}[\mathrm{M} 10]$ & $16(9)$ & $\mathrm{C}^{-1} \mathrm{C}[\mathrm{M} 9]$ & $14(8)$ \\
\hline $\mathrm{C}^{-2}$ & $\mathrm{C}^{-2} \mathrm{C}[\mathrm{M} 12]$ & $17(10)$ & $\mathrm{C}^{-2} \mathrm{C}[\mathrm{M} 11]$ & $15(9)$ & $\mathrm{C}^{-2} \mathrm{C}[\mathrm{M} 10]$ & $13(8)$ & $\mathrm{C}^{-2} \mathrm{C}[\mathrm{M} 9]$ & $11(7)$ \\
\hline $\mathrm{C}^{-3}$ & $\mathrm{C}^{-3} \mathrm{C}[\mathrm{M} 12]$ & $14(9)$ & $\mathrm{C}^{-3} \mathrm{C}[\mathrm{M} 11]$ & $12(8)$ & $\mathrm{C}^{-3} \mathrm{C}[\mathrm{M} 10]$ & $10(7)$ & $\mathrm{C}^{-3} \mathrm{C}$ [M9] & $8(6)$ \\
\hline $\mathrm{C}^{-4}$ & $\mathrm{C}^{-4} \mathrm{C}[\mathrm{M} 12]$ & $11(8)$ & $\mathrm{C}^{-4} \mathrm{C}[\mathrm{M} 11]$ & $9(7)$ & $\mathrm{C}^{-4} \mathrm{C}[\mathrm{M} 10]$ & $7(6)$ & $\mathrm{C}^{-4} \mathrm{C}[\mathrm{M9}]$ & $5(5)$ \\
\hline$C^{-5}$ & $\mathrm{C}^{-5} \mathrm{C}[\mathrm{M} 12]$ & $8(7)$ & $\mathrm{C}^{-5} \mathrm{C}[\mathrm{M} 11]$ & $6(6)$ & $\mathrm{C}^{-5} \mathrm{C}[\mathrm{M} 10]$ & $4(5)$ & $\mathrm{C}^{-5} \mathrm{C}[\mathrm{M} 9]$ & 2(4) \\
\hline $\mathrm{C}^{-6}$ & $\mathrm{C}^{-6} \mathrm{C}[\mathrm{M12}]$ & $5(6)$ & $\mathrm{C}^{-6} \mathrm{C}[\mathrm{M} 11]$ & $3(5)$ & & & & \\
\hline $\mathrm{C}^{-7}$ & $\mathrm{C}^{-7} \mathrm{C}[\mathrm{M} 12]$ & $2(5)$ & $\mathrm{C}^{-7} \mathrm{C}[\mathrm{M} 11]$ & $0(4)$ & & & & \\
\hline
\end{tabular}

\begin{tabular}{lllllll}
\hline & {$[\mathrm{M} 8]$} & $\mathrm{K}(\mathrm{n})$ & {$[\mathrm{M} 7]$} & $\mathrm{K}(\mathrm{n})$ & {$[\mathrm{M} 7]$} & $\mathrm{K}(\mathrm{n})$ \\
\hline $\mathbf{C}^{\mathbf{0}}$ & $\mathbf{C}^{0}[\mathbf{M} 8]$ & $\mathbf{1 5}(\mathbf{8})$ & $\mathbf{C}^{0}[\mathbf{M} 7]$ & $\mathbf{1 3}(\mathbf{7})$ & $\mathbf{C}^{0}[\mathbf{M} 7]$ & $\mathbf{1 3}(\mathbf{7})$ \\
$\mathrm{C}^{-1}$ & $\mathrm{C}^{-1}[\mathrm{M} 8]$ & $12(7)$ & $\mathrm{C}^{-1}[\mathrm{M} 7]$ & $10(6)$ & $\mathrm{C}^{-1}[\mathrm{M} 7]$ & $10(6)$ \\
$\mathrm{C}^{-2}$ & $\mathrm{C}^{-2}[\mathrm{M} 8]$ & $9(6)$ & $\mathrm{C}^{-2}[\mathrm{M} 7]$ & $7(5)$ & $\mathrm{C}^{-2}[\mathrm{M} 7]$ & $7(5)$ \\
$\mathrm{C}^{-3}$ & $\mathrm{C}^{-3}[\mathrm{M} 8]$ & $6(5)$ & $\mathbf{C}^{-3}[\mathbf{M} 7]$ & $\mathbf{4 ( 4 )}$ & $\mathbf{C}^{-3}[\mathrm{M} 7]$ & $\mathbf{4 ( 4 )}$ \\
$\mathrm{C}^{-4}$ & $\mathbf{C}^{-4}[\mathbf{M} 8]$ & $\mathbf{3 ( 4 )}$ & $\mathrm{C}^{-4}[\mathrm{M} 7]$ & $1(3)$ & $\mathrm{C}^{-4}[\mathrm{M} 7]$ & $1(3)$ \\
\hline
\end{tabular}

\begin{tabular}{lllllllllll}
\hline & {$[\mathrm{M} 6]$} & $\mathrm{K}(\mathrm{n})$ & {$[\mathrm{M} 5]$} & $\mathrm{K}(\mathrm{n})$ & {$[\mathrm{M} 4]$} & $\mathrm{K}(\mathrm{n})$ & {$[\mathrm{M} 3]$} & $\mathrm{K}(\mathrm{n})$ & {$[\mathrm{M} 2]$} & $\mathrm{K}(\mathrm{n})$ \\
\hline $\mathbf{C}^{\mathbf{0}}$ & $\mathbf{C}^{0}[\mathbf{M 6}]$ & $\mathbf{1 1}(\mathbf{6})$ & $\mathbf{C}^{0}[\mathbf{M 5}]$ & $\mathbf{9 ( 5 )}$ & $\mathbf{C}^{0}[\mathbf{M 4}]$ & $\mathbf{7 ( 4 )}$ & $\mathbf{C}^{0}[\mathbf{M 3}]$ & $\mathbf{5 ( 3 )}$ & $\mathbf{C}^{0}[\mathbf{M} 2]$ & $\mathbf{3}(\mathbf{2})$ \\
$\mathrm{C}^{-1}$ & $\mathrm{C}^{-1}[\mathrm{M} 6]$ & $8(5)$ & $\mathrm{C}^{-1}[\mathrm{M} 5]$ & $6(4)$ & $\mathrm{C}^{-1}[\mathrm{M} 4]$ & $4(3)$ & $\mathbf{C}^{-1}[\mathbf{M 3}]$ & $\mathbf{2 ( 2 )}$ & $\mathbf{C}^{-1}[\mathbf{M} 2]$ & $\mathbf{0}(\mathbf{1})$ \\
$\mathrm{C}^{-2}$ & $\mathrm{C}^{-2}[\mathrm{M} 6]$ & $5(4)$ & $\mathbf{C}^{-2}[\mathbf{M 5}]$ & $\mathbf{3 ( 3 )}$ & $\mathbf{C}^{-2}[\mathbf{M 4}]$ & $\mathbf{1}(\mathbf{2})$ & & & & \\
$\mathrm{C}^{-3}$ & $\mathbf{C}^{-3}[\mathbf{M 6}]$ & $\mathbf{2 ( 3 )}$ & $\mathrm{C}^{-3}[\mathrm{M} 5]$ & $0(2)$ & & & & & & \\
\hline
\end{tabular}

Table 5. Some of Rudolph Type of Series and Corresponding Boran

\begin{tabular}{llllllll}
\hline$[\mathbf{M 1 2}]$ & $\mathbf{K}(\mathbf{n})$ & $\mathbf{S}$ & $\mathbf{F}_{\mathbf{B}}$ & {$[\mathrm{M} 6]$} & $\mathrm{K}(\mathrm{n})$ & $\mathbf{S}$ & $\mathbf{F}_{\mathbf{B}}$ \\
\hline $\mathrm{C}^{0} \mathrm{C}[\mathrm{M} 12]$ & $23(12)$ & $4 \mathrm{n}+2$ & {$[\mathrm{BH}](12)+2 \mathrm{e}=\mathrm{B}_{12} \mathrm{H}_{12}{ }^{2-}$} & $\mathrm{C}^{0}[\mathrm{M} 6]$ & $11(6)$ & $4 \mathrm{n}+2$ & {$[\mathrm{BH}](6)+2 \mathrm{e}=\mathrm{B}_{6} \mathrm{H}_{6}{ }^{2}-$} \\
$\mathrm{C}^{-1} \mathrm{C}[\mathrm{M} 12]$ & $20(11)$ & $4 \mathrm{n}+4$ & {$[\mathrm{BH}](11)+4 \mathrm{H}=\mathrm{B}_{11} \mathrm{H}_{15}$} & $\mathrm{C}^{-1}[\mathrm{M} 6]$ & $8(5)$ & $4 \mathrm{n}+4$ & {$[\mathrm{BH}](5)+4 \mathrm{H}=\mathrm{B}_{5} \mathrm{H}_{9}$} \\
$\mathrm{C}^{-2} \mathrm{C}[\mathrm{M} 12]$ & $17(10)$ & $4 \mathrm{n}+6$ & {$[\mathrm{BH}](10)+6 \mathrm{H}=\mathrm{B}_{10} \mathrm{H}_{16}$} & $\mathrm{C}^{-2}[\mathrm{M} 6]$ & $5(4)$ & $4 \mathrm{n}+6$ & {$[\mathrm{BH}](4)+6 \mathrm{H}=\mathrm{B}_{4} \mathrm{H}_{10}$} \\
$\mathrm{C}^{-3} \mathrm{C}[\mathrm{M} 12]$ & $14(9)$ & $4 \mathrm{n}+8$ & {$[\mathrm{BH}](9)+8 \mathrm{H}=\mathrm{B}_{9} \mathrm{H}_{17}$} & $\mathbf{C}^{-3}[\mathbf{M} 6]$ & $\mathbf{2 ( 3 )}$ & $4 \mathrm{n}+8$ & {$[\mathrm{BH}](3)+8 \mathrm{H}=\mathrm{B}_{3} \mathrm{H}_{11}$} \\
$\mathrm{C}^{-4} \mathrm{C}[\mathrm{M} 12]$ & $11(8)$ & $4 \mathrm{n}+10$ & {$[\mathrm{BH}](8)+10 \mathrm{H}=\mathrm{B}_{8} \mathrm{H}_{18}$} & & & & \\
$\mathrm{C}^{-5} \mathrm{C}[\mathrm{M} 12]$ & $8(7)$ & $4 \mathrm{n}+12$ & {$[\mathrm{BH}](7)+12 \mathrm{H}=\mathrm{B}_{7} \mathrm{H}_{19}$} & & & & \\
$\mathbf{C}^{-\mathbf{6}} \mathbf{C}[\mathbf{M 1 2}]$ & $\mathbf{5 ( 6 )}$ & $\mathbf{4 n + 1 4}$ & {$[\mathbf{B H}](\mathbf{6})+\mathbf{1 4 H}=\mathbf{B}_{\mathbf{6}} \mathbf{H}_{\mathbf{2 0}}$} & & & & \\
\hline
\end{tabular}


$\mathrm{P}_{4} \quad: \mathrm{K}=4[1.5]=6$

$\underset{\substack{K(n)=6(4) \\ \mathrm{S}=4 \mathrm{n}+4(\mathrm{q}=4)}}{\stackrel{-1(2)}{\longrightarrow} \mathrm{q}} \mathrm{q}=2$

$\operatorname{Ir}_{4}(\mathrm{CO}){ }_{12} \quad: \mathrm{K}=4[4.5]-12=6$

$\begin{array}{ll}\mathrm{K}(\mathrm{n})=6(4) & -1(2) \\ \mathrm{S}=4 \mathrm{n}+4 & \\ \mathrm{n}=4 & \downarrow\end{array}$

$\mathrm{Kp}=\mathrm{C}^{-1} \mathrm{C}[\mathrm{M} 5]$

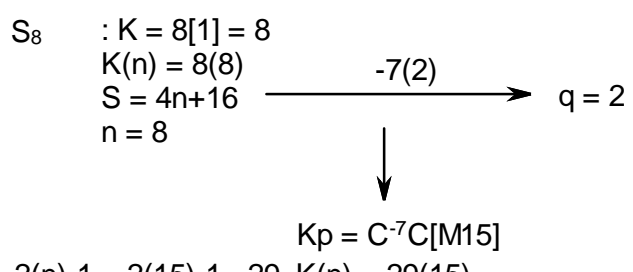

[M15], $\mathrm{K}=2(\mathrm{n})-1=2(15)-1=29, \mathrm{~K}(\mathrm{n})=29(15)$

$\begin{array}{llllll}29(15) & 26(14) & 23(13) & 20(12) & 17(11) & 14(10)\end{array} \quad$ 11(9)

SC-8

Appendix-1

Appendix-2

\begin{tabular}{|c|c|c|c|c|c|c|c|}
\hline \multicolumn{8}{|c|}{ Skeletal k Values of Main Group Elements } \\
\hline Group & Series, $S=4 n+q$ & $k=2 n-q / 2$ & & & & & \\
\hline 1 & $4 n-3$ & 3.5 & $\mathbf{L i}$ & $\mathbf{N a}$ & $\mathbf{K}$ & $\mathbf{R b}$ & Cs \\
\hline 2 & $4 n-2$ & 3 & Be & Mg & $\mathbf{C a}$ & $\mathbf{S r}$ & Ba \\
\hline 3 & 4n-1 & 2.5 & B & Al & $\mathbf{G a}$ & In & Tl \\
\hline 4 & $4 n+0$ & 2 & $\mathbf{C}$ & Si & Ge & Sn & $\mathbf{P b}$ \\
\hline 5 & $4 n+1$ & 1.5 & $\mathbf{N}$ & $\mathbf{P}$ & As & Sb & Bi \\
\hline 6 & $4 n+2$ & 1 & O & $\mathbf{S}$ & Se & $\mathbf{T e}$ & Po \\
\hline 7 & $4 n+3$ & 0.5 & $\mathbf{F}$ & Cl & $\mathbf{B r}$ & I & At \\
\hline 8 & $4 n+4$ & 0 & $\mathrm{Ne}$ & Ar & $\mathbf{K r}$ & $\mathrm{Xe}$ & Rn \\
\hline
\end{tabular}

\begin{tabular}{ccccc}
\hline \multicolumn{5}{c}{ Skeletal k Values of Transition Metals } \\
\hline 3d-TM & 4d - & 5d-TM & Series, S & k =2n-q/2 \\
$*$ & TM* & $*$ & $4 n+q$ & \\
Sc & Y & La & $4 n-11$ & 7.5 \\
Ti & Zr & Hf & $4 n-10$ & 7 \\
V & Nb & Ta & $4 n-9$ & 6.5 \\
Cr & Mo & W & $4 n-8$ & 6 \\
Mn & Tc & Re & $4 n-7$ & 5.5 \\
Fe & Ru & Os & $4 n-6$ & 5 \\
Co & Rh & Ir & $4 n-5$ & 4.5 \\
Ni & Pd & Pt & $4 n-4$ & 4 \\
Cu & Ag & Au & $4 n-3$ & 3.5 \\
Zn & Cd & Hg & $4 n-2$ & 3 \\
\hline
\end{tabular}

\section{Conclusion}

A collection of known golden clusters have been analyzed and categorized using skeletal numbers. A large percentage of them are centered around [M0], [M1] and [M2] closo-based axes. The [Mx] may be regarded as similar to groups of elements in the periodic table. The clusters which lie on the closo lines $[\mathrm{Mx}], \mathrm{x} \leq 0$, have a tendency of possessing metallic character. In this connection, the golden clusters are closer to the metallic region of metallic clusters than that of non-metallic. A concept of a black-hole nucleus has been introduced as well as that of numerical categorization of clusters which lie lower than the closo base line. The two dimensional ideal shapes of clusters have been sketched using graph theory derived from the $4 \mathrm{n}$ series method. 


\section{Acknowledgement}

My gratitude is extended to Dr. P.E.T. Kiremire for editing the draft and Mrs. Merab Kambamu Kiremire for the continued encouragement to write these articles.

\section{References}

Corti, C. W., Holliday, R. J., \& Thompson, D. J. (2005). Commercial Aspects of Gold Catalysis A. General, 291, 253-261. https://doi.org/10.1016/j.apcata.2005.01.051

Cotton, F. A., \& Wilkinson, G. (1980). Advanced Inorganic Chemistry, $4^{\text {th }}$ Ed., John Wiley and Sons, New York, 1980.

Gimeno, M. C. (2008). Modern Supramolecular Gold-Metal Interactive and Applications. Edited A. Laguna, 2008. Wiley-VCH, Weinheim.

Greenwood, N. N., \& Earnshaw, A. (1998). Chemistry of the Elements, $2^{\text {nd }} E d$. Butterworth, Oxford.

Hughes, A. K., \& Wade, K. (2000). Metal-metal and metal-ligand strengths in metal carbonyl clusters. Coord. Chem. Rev., 197, 191-229. https://doi.org/10.1016/S0010-8545(99)00208-8

Kilmartin, J. (2010). Molecular Gold Clusters as Precursors to Heterogeneous Catalysis. PhD Thesis.

Kiremire, E. M. R. (2015). Capping and Decapping Series of Boranes. Int. J. Chem., 7(2), 186-197. https://doi.org/10.5539/ijc.v7n2p186

Kiremire, E. M. R. (2016a). Clusters of Gold Containing P-Block Elements. Am. J. Chem., 6(5), 126-144.

Kiremire, E. M. R. (2016b). Unusual underground capping Carbonyl Clusters of Palladium. Int. J. Chem., 8(1), 145-158https://doi.org/10.5539/ijc.v8n1p145

Kiremire, E. M. R. (2017a). The Golden Series and Clusters of Gold-unique Shapes and Bonding. Int. J. Chem., 9(1), 38-57. https://doi.org/10.5539/ijc.v9n1p38

Kiremire, E. M. R. (2017b). The Six Silent Laws of Chemical Clusters. Am. J. Chem., 7(2), 21-41.

Kiremire, E. M. R. (2017c). Boranes, Metalloboranes, Transition Metal Carbonyls and Other Cluster Formulas Obey the Law of Skeletal Numbers and Their Valences. Am. J. Chem., 7(4), 113-144.

Kiremire, E. M. R. (2017d). Numerical Characterization of Fragments, Molecules, and Clusters Using Skeletal Numbers and Nuclearity Trees. Am. J. Chem.,7(3),73-96.

Konishi, K. (2014). Phosphine-Coordinated Pure-Gold Clusters and Unique Optical Properties /Responses. Structure and Bonding, 161, 49-86. https://doi.org/10.1007/430_2014_143

Kwok-Ming, L. (2011). Synthesis, characterization and photophysical properties of Chalcogenido, phosphinidene and alkyl Complexes of Gold(I) and its Congener and their Supramolecular assembly arising from metal-metal interactions. PhD Thesis.

Mingos, D. M. P. (1984). Gold Cluster Compounds: Are they materials in miniature? Gold Bull., 17(1), 5-12. https://doi.org/10.1007/BF03214670

Rudolph, R. W.(1976). Boranes and heteroboranes: a paradigm for the electron requirements of clusters? Acc. Chem. Res., 9(12), 446-452. https://doi.org/10.1021/ar50108a004

Zhang, Q. F., Williard, P. G., \& Wang, L. S. (2016). Polymorphism of Phosphine-Protected Gold nano-clusters: Synthesis A, Weinheim.

Zhang, Y., Chu, W., Foroushani, A. D., Wang, H., Li, D., Liu, J., Barrow, C. J., Wang, X., \& Yang, W. (2014). New Gold Nanostructures for Sensor Applications. A Review. Materials, 7, 5169-5201. https://doi.org/10.3390/ma7075169

\section{Copyrights}

Copyright for this article is retained by the author(s), with first publication rights granted to the journal.

This is an open-access article distributed under the terms and conditions of the Creative Commons Attribution license (http://creativecommons.org/licenses/by/4.0/). 